\author{
Dissertation \\ submitted to the \\ Combined Faculties for the Natural Sciences and for Mathematics \\ of the Ruperto-Carola University of Heidelberg, Germany \\ for the degree of \\ Doctor of Natural Sciences
}

presented by

Diplom-Ing. (FH) / MSc. : $\quad$ Patrick Theer

Born in:

Berlin

Oral examination: 30.06.2004 


\section{On the fundamental imaging-depth limit in two-photon microscopy.}

Referees:

Prof. Dr. Winfried Denk

Prof. Dr. Josef Bille 


\title{
Zusammenfassung
}

Einer der grundlegenden Vorteile der Zwei-Photonen Mikroskopie gegenüber Ein-Photonen Techniken ist die Möglichkeit der Aufnahme hochauflösender Bilder tief in lebenden Geweben. Obwohl Bildtiefen von $500 \mu \mathrm{m}$ in Gehirngewebe heutzutage Standard sind, sind größere Tiefen aufgrund der limitierten optischen Leistung herkömmlicher Femto-Sekunden Laser unzugänglich gewesen. In dieser Arbeit werden Strategien zur Verbesserung der Bildtiefe in der Zwei-Photonen Mikroskopie untersucht. Im Speziellen wird gezeigt, daß, mittels optisch verstärkter Laser Pulse, signifikante Verbesserungen der Bildtiefe möglich sind. Unter Benutzung eines regenerativen Laserverstärkers, wurden Bilder von gefärbten Gefäßen und Neuronen im lebenden Gehirn von Mäusen bis zu Tiefen von bis zu $1000 \mu \mathrm{m}$ aufgenommen. In diesen Experimenten war die maximale Bildtiefe nicht mehr durch die maximal verfügbare Laserleistung limitiert sondern durch eine Zunahme in der Hintergrundfluoreszenz. Um dieses Verhalten quantitativ zu beschreiben, wurde der Einfluß der Lichtstreuung auf die Anregung und Detektion von Fluoreszenz untersucht. Die Parameter mit dem größten Einfluß auf die maximal erreichbare Bildtiefe sind die Numerische Apertur und die Färbecharakteristik des Untersuchungsobjektes. Die größten Bildtiefen werden mit der größten numerischen Apertur und der geringsten Hintergrundfärbung des Untersuchungsobjektes erzielt.

\begin{abstract}
One of the principle advantages of two-photon microscopy over one-photon techniques is that it can provide high-resolution images from very deep within living tissue. While imaging depths of $500 \mu \mathrm{m}$ in brain tissue have become standard performance, larger depths have been inaccessible mainly due to the power limitation of current femto-second laser sources. Here we investigate strategies to improve the imaging depth in two-photon microscopy. In particular, we show that the two-photon imaging depth can be significantly improved using optically amplified femtosecond laser pulses. Using a regenerative amplifier as the excitation source we obtained images of stained vasculature and GFP-labeled neurons down to a depth of about $1000 \mu \mathrm{m}$ below the brain surface in the cortex of mice in vivo. The maximum imaging depth was now limited by outof-focus background fluorescence and not by the available excitation power. In order to provide a quantitative description of this behavior, we have investigated the effects of scattering on fluorescence excitation and detection. The most prominent parameters that influence the maximum two-photon imaging depth are the excitation numerical aperture and the sample staining characteristics. The largest depths can be achieved with the largest excitation numerical aperture and the lowest out-of-focus volume staining.
\end{abstract}




\section{Contents}

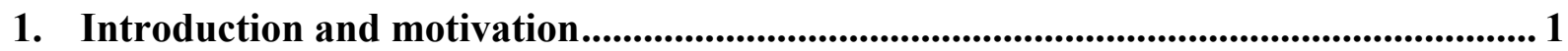

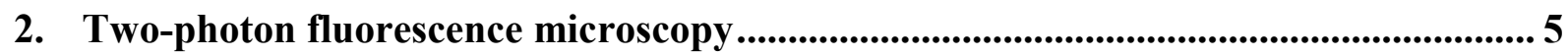

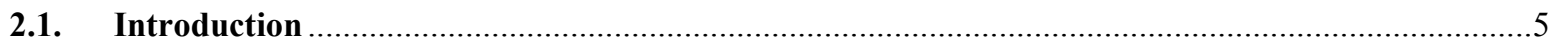

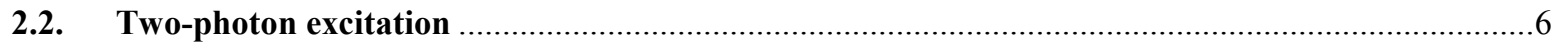

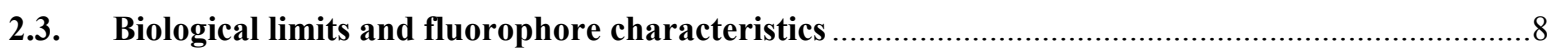

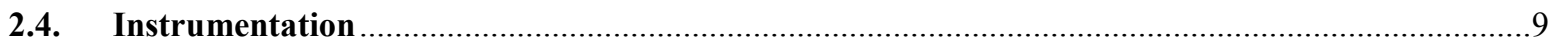

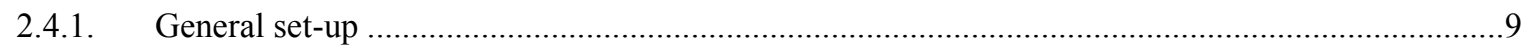

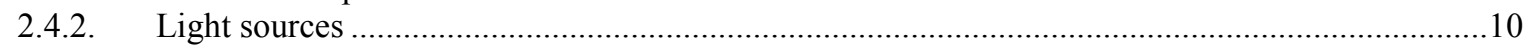

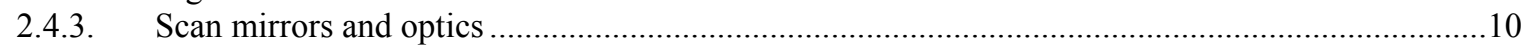

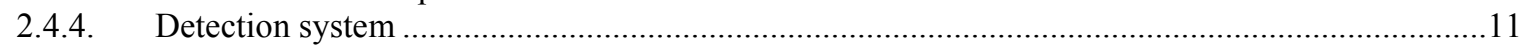

3. Imaging depth in two-photon fluorescence microscopy ................................................ 15

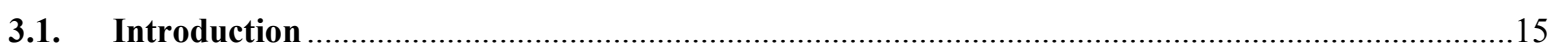

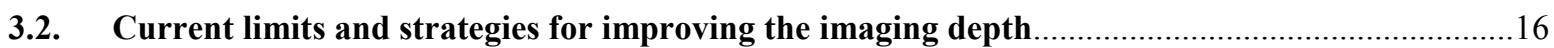

3.3. Regenerative amplification and its applicability for two-photon microscopy …….......................17

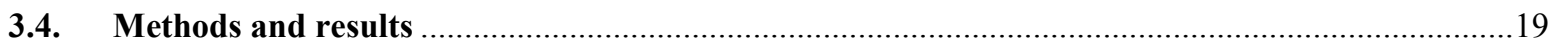

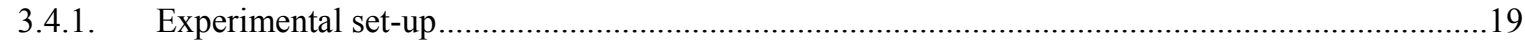

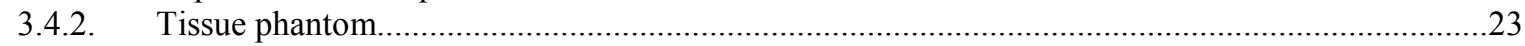

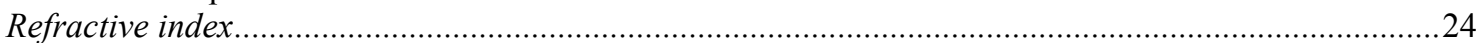

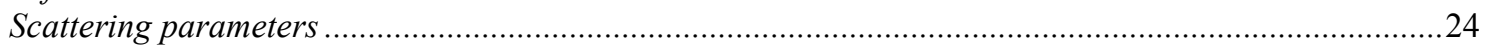

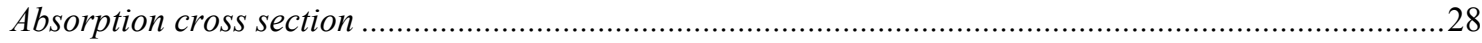

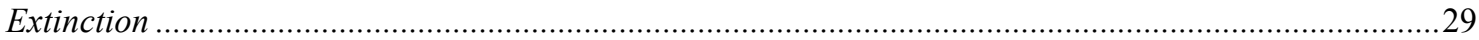

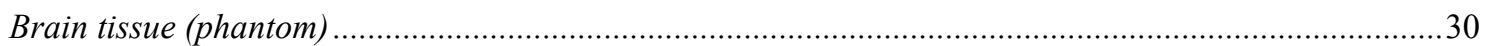

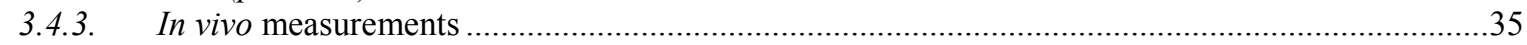

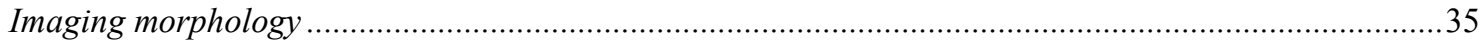

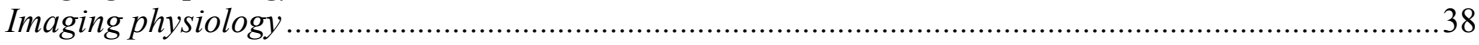

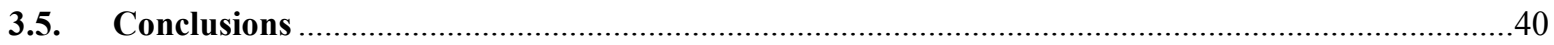

4. On the fundamental imaging-depth limit in two-photon microscopy.........................45

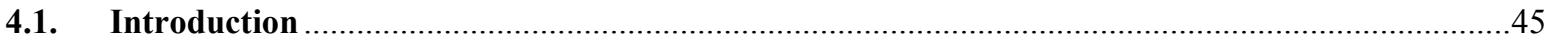

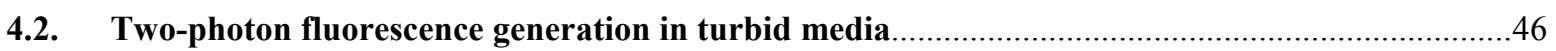

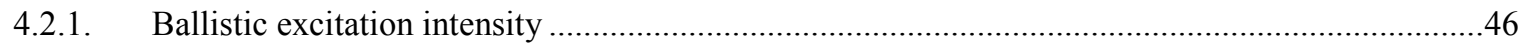

4.2.2. Effective waist and far-field intensity for a truncated Gaussian beam ..........................................51

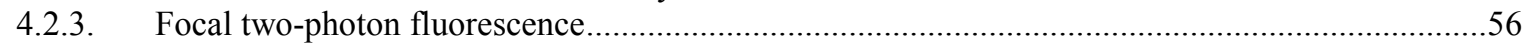

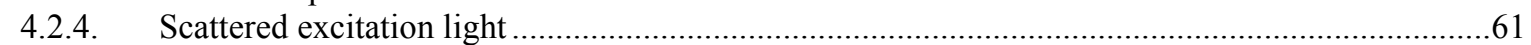

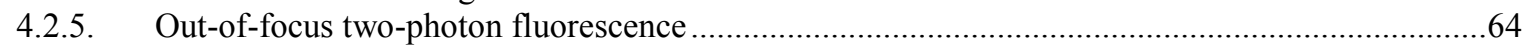

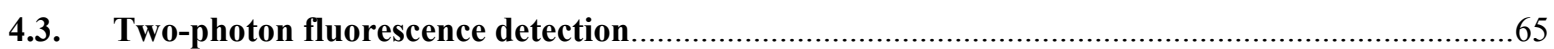




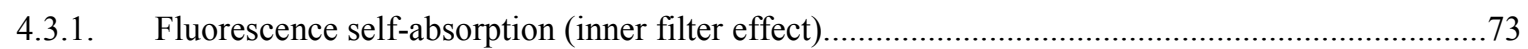

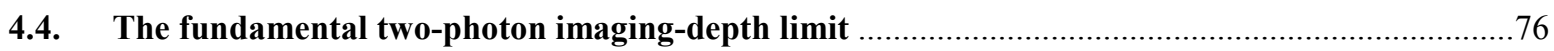

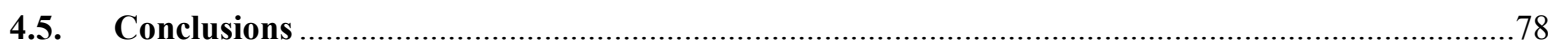

5. Strategies for extending the imaging depth beyond the depth limit ........................... 81

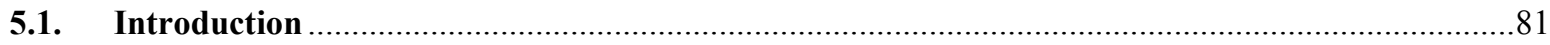

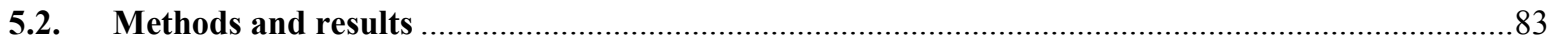

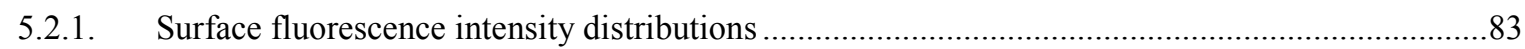

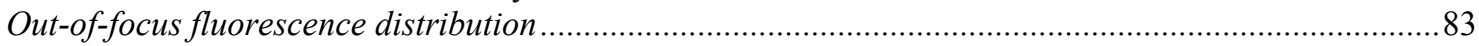

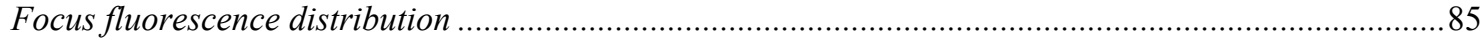

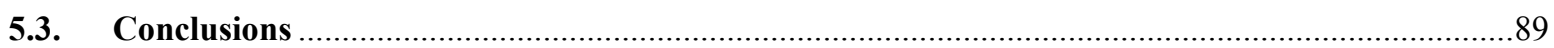

6. Summary …............................................................................................................................. 91

7. Acknowledgements ................................................................................................................ 93

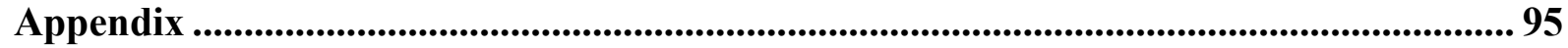

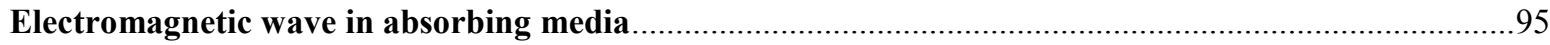




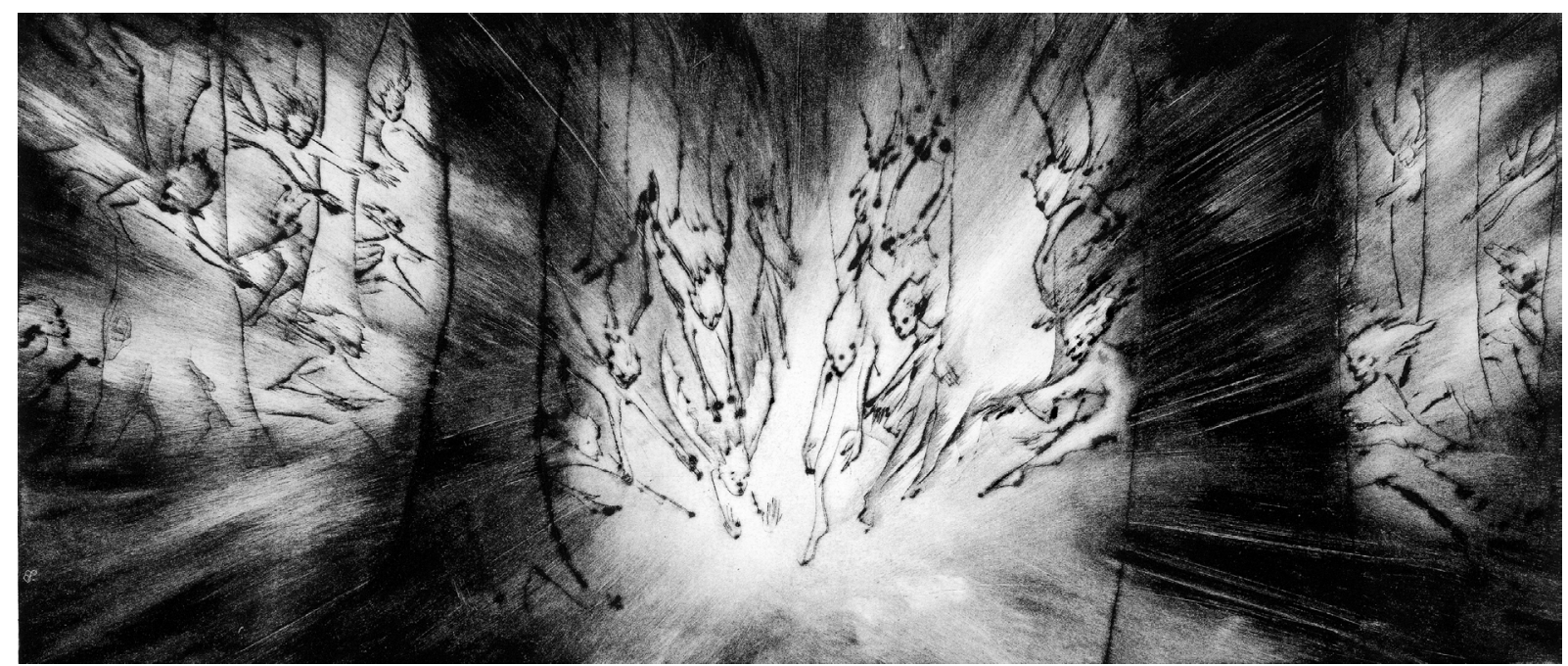

Just how many of them can you lure this deep?

bre a 2004

\section{Introduction and motivation}

Ever since Antoni van Leeuwenhoek's superb use of the light microscope to discover the world of micro organisms ${ }^{1}$ optical imaging has been one of the core technologies in biology. However, within the last three decades light microscopy has begun a remarkable transformation. Recent technical advances in illumination sources and detectors, computational tools and developments in organic chemistry and molecular biology resulted in microscopy evolving into a modern endeavour playing a central role in a wide spectrum of disciplines. One of the most rapidly expanding microscopy techniques employed today is fluorescence microscopy. It is based on the property of some atoms and molecules to absorb light at a particular wavelength and to subsequently emit light of longer wavelength after a brief interval (fluorescence lifetime). Fluorescence microscopy is thus capable of imaging the 
distribution of a single molecular species based solely on the properties of fluorescence excitation and emission. It is, however, frequently limited in its sensitivity and spatial resolution due to out-of-focus fluorescence. This problem has been partly solved with the introduction of the confocal microscope in the 1980's. The key to the confocal approach, proposed by Marvin Minsky in $1957,{ }^{2}$ is the use of spatial filtering to inhibit the detection of scattered and out-of-focus light in specimens that are thicker than the plane of focus. This, for the first time, allowed the three-dimensional visualisation of living specimens on a microscopic scale. One of the major drawbacks of confocal microscopy is the inefficient use of excitation. While information is obtained only from the focal volume, fluorescence - and hence photobleaching and photodamage - is generated all along the illumination path. In addition, of all the fluorescence generated in the focal volume only photons that leave the specimen unscattered (in thick scattering specimens a small minority) will be detected. Both the inefficient use of signal fluorescence and the exposure to photobleaching and damage of the out-of-focus volume restrict confocal microscopy to small imaging depths.

Virtually all of these problems have been solved with the invention of the two-photon fluorescence microscope in $1990 .^{3}$ This technique is based on the nearly simultaneous absorption of two photons promoting an electronic transition that would otherwise require a single photon of twice their energy. The probability for such an event is extremely low at ambient intensities and occurs at appreciable rates only at very high intensities (in general > $10^{17} \mathrm{~W} / \mathrm{m}^{2}$ ). Such intensities can usually only be achieved in the focus of a high-NA lens using a mode-locked laser-source with sub-picosecond pulse duration. Two-photon absorption is thus confined to the focal volume which provides inherent optical sectioning without the use of a spatial filter. Unlike the confocal microscopes this allows the detection of scattered fluorescence photons. Both the detection of scattered fluorescence and the reduced scattering cross-section (increased scattering length) for low energy photons (usually in the infrared) contribute to the capability of the two-photon microscope to provide high resolution images from very deep within living tissue. ${ }^{4,5}$ Imaging to a depth of 2-3 scattering mean-free-path lengths has become standard performance. Compared to what can be achieved with confocal microscopy this constitutes a three-fold increase in imaging depth. However, imaging at larger depths has been restricted by the maximum power unamplified lasers can provide. Efforts to increase the imaging depth have since concentrated on increasing the detection and excitation efficiency. These studies have revealed the importance of using lenses with high numerical apertures and a large field-of-view ${ }^{6}$ as well as ultra short laser pulses ${ }^{7}$ at low repetition rates ${ }^{8}$ 
and they served as the basis for the work presented in this dissertation. It has, however, also become clear that the imaging depth in two-photon microscopy cannot be increased indefinitely by increasing excitation power and efficiency but is fundamentally limited by the onset of out-of-focus fluorescence generation near the top of the sample, ${ }^{9}$ since for very thick absorbing or scattering samples the assumption that two-photon fluorescence is largely confined to the focal region is no longer true.

The goal of this work is to increase the imaging depth in two-photon microscopy by increasing the two-photon detection and excitation efficiency with a main focus on increasing the excitation efficiency through the use of optically amplified laser pulses. The chapters 2 and 3 of this dissertation will introduce the physical principles relevant to two-photon fluorescence microscopy and how they relate to the actual instrumentation, discuss the limits pertaining to the two-photon imaging depth in biological specimens and present strategies suitable to improve this depth. Emphasis will be put on the use of optically amplified laser pulses as the most promising means for increasing the imaging depth. Following an introduction to the concept of regenerative amplification and a discussion on its applicability for two-photon fluorescence microscopy, the third chapter will end with demonstrating its feasibility for in vivo measurements and show that a substantial increase in imaging depth can indeed be achieved. In the fourth chapter, the findings of the initial measurements will be put on a firm theoretical basis by investigating two-photon fluorescence excitation and detection in turbid media. Careful analysis of the role of scattered excitation light on the generation of two-photon fluorescence revealed the surprising result that scattered excitation light accounts for a substantial part of the out-of-focus fluorescence. Strategies for further improving the imaging depth and advancing its fundamental limit will be presented in chapter five.

\section{References}

1. A. van Leeuwenhoek, in The collected Letters of Antoni van Leeuwenhoek (Swets \& Zeitlinger, Amsterdam, 1939-).

2. $\quad$ M. Minsky, "Microscopy Apparatus," U.S. Patent \#3013467 (1957).

3. W. Denk, J. H. Strickler, and W. W. Webb, "2-Photon Laser Scanning Fluorescence Microscopy," Science 248(4951), 73-76 (1990). 
4. W. Denk and K. Svoboda, "Photon upmanship: Why multiphoton imaging is more than a gimmick," Neuron 18(3), 351-357 (1997).

5. W. R. Zipfel, R. M. Williams, and W. W. Webb, "Nonlinear magic: multiphoton microscopy in the biosciences," Nature Biotechnology 21(11), 1368-1376 (2003).

6. M. Oheim, E. Beaurepaire, E. Chaigneau, J. Mertz, and S. Charpak, "Two-photon microscopy in brain tissue: parameters influencing the imaging depth," Journal of Neuroscience Methods 111(1), 29-37 (2001).

7. M. Muller, J. Squier, R. Wolleschensky, U. Simon, and G. J. Brakenhoff, "Dispersion pre-compensation of 15 femtosecond optical pulses for high-numerical-aperture objectives," Journal of Microscopy-Oxford 191, 141-150 (1998).

8. E. Beaurepaire, M. Oheim, and J. Mertz, "Ultra-deep two-photon fluorescence excitation in turbid media," Optics Communications 188(1-4), 25-29 (2001).

9. J. P. Ying, F. Liu, and R. R. Alfano, "Spatial distribution of two-photon-excited fluorescence in scattering media (vol 38, pg 224, 1999)," Applied Optics 38(10), 2151 2151 (1999). 


\section{Two-photon fluorescence microscopy}

\subsection{Introduction}

Since its inception more than a decade ago, ${ }^{1}$ two-photon fluorescence microscopy has been widely used in the field of biology and medicine. One of its major advantages over onephoton techniques is that as a result of the nonlinear character of two-photon excitation, it provides inherent sectioning capability - confining excitation to the high-intensity region at the focus. This not only reduces photobleaching and damage in the out-of-focus volume but, in addition, allows for a significant increase in detection efficiency.

Two-photon excitation as a single quantum event is accomplished by the simultaneous absorption of two photons, each having approximately half the energy required to cause a transition to the excited state of the fluorophore (see Figure 2.1).

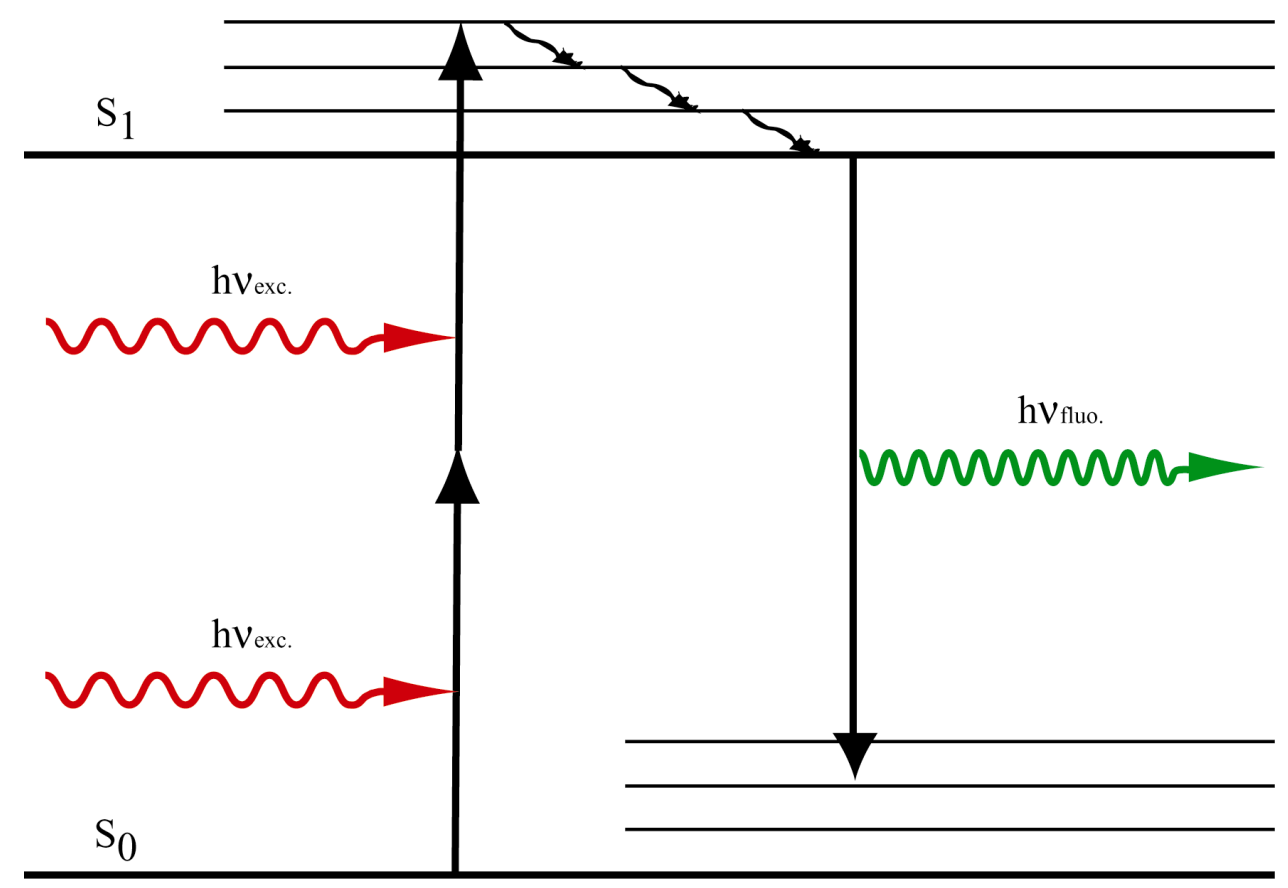

Fig. $2.1 \quad$ Principle of two-photon excitation. A molecule in the ground state $\left(\mathrm{S}_{0}\right)$ is excited (here to a vibrational level above the first excited state $S_{1}$ ) by simultaneous absorption of two low energy photons. It relaxes down the vibrational ladder to $S_{1}$ and usually returns to a vibrational level above $S_{0}$ via emission of one high energy fluorescence photon $\left(h v_{\text {fluo. }} \leq 2 \mathrm{~h} v_{\text {exc. }}\right)$. 
Although the theoretical basis had already been established in 1931 by Maria Göppert-Mayer ${ }^{2}$ the experimental observation ${ }^{3}$ of two-photon excitation had to wait for the invention of the laser because of the large light intensities required. Its value for microscopy was not recognized until $1990 .^{1}$

This chapter aims to introduce the physical principles governing two-photon fluorescence excitation microscopy and how they relate to the actual instrumentation, and to discuss limitations to biological and medical research. Much of the content of this chapter has been elaborated in parallel with a manuscript (co-authors Axel Nimmerjahn and Fritjof Helmchen) “Two-Photon Laser Scanning Microscopy" invited for publication in an edited multiauthor book on "Ultrashort Laser Pulses in Biology and Medicine" (Springer publishing house, Heidelberg).

\subsection{Two-photon excitation}

Two-photon excitation requires the absorption of two photons within a very narrow temporal window, typically less than $10^{-15} \mathrm{~s}$ as defined by the Heisenberg uncertainty principle. The absorption cross section $\sigma$ describing this process depends linearly on the excitation intensity i.e. $\sigma=\delta I$ where $\delta$ is the two-photon absorption cross-section, measured in units of GöppertMayer ( $1 \mathrm{GM}=10^{-58} \mathrm{~m}^{4} \mathrm{~s} /$ photon). The two-photon absorption rate thus scales with the square of the excitation intensity

$$
R_{a b s}=\sigma I /(h c \lambda)=\delta I^{2} /(h c \lambda)
$$

where $h, c$, and $\lambda$ are the Planck constant, speed of light in vacuum, and the wavelength respectively.

Owing to the square dependence on the light intensity, significant two-photon absorption rates require very high photon flux densities in the range of $\mathrm{GW} / \mathrm{cm}^{2}$. These can be achieved by temporal and spatial concentration of laser light. Although some two-photon excitation using spatial confinement alone, by using high numerical aperture lenses, has been demonstrated, ${ }^{4}$ acceptable average power levels and time-efficient imaging generally requires additional temporal concentration through the use of pulsed radiation. Under typical experimental conditions i.e. fluorescence excitation using a focused, pulsed laser beam, the average number of photon pairs absorbed per fluorophore and per unit time is given by ${ }^{1}$ 


$$
n_{a}=\frac{\delta}{\tau f}\left(\frac{\pi(N A)^{2}}{h c \lambda}\right)^{2}\langle P\rangle^{2}
$$

where $\langle P\rangle$ is the average power, $N A$ is the numerical aperture, and $f$ and $\tau$ are the pulse repetition rate and duration respectively ${ }^{\dagger}$.

According to Eqn. (2.2), the two-photon fluorescence yield can be increased by decreasing the repetition frequency or the pulse duration (assuming constant average power). This is, however, not generally true for several reasons. First, pulses propagating through optical glass and coatings are spread in time due to group velocity dispersion (GVD). This stems from the fact that the light of ultra-short pulses consists of a relative wide range of optical frequencies, which travel, in most optical materials, at different speeds (group velocities). Since the difference in speed increases with the spectral width of the pulse, shortening of the pulse duration which is associated with an increase of the spectral width, leads to chirped (frequency swept) pulses which are longer than the originals. Although pulse spreading by GVD can be compensated by pre-chirping, e.g. using prism or grating arrangements, for providing pulse widths much smaller than 100 fs in the focus of high NA objectives, compensation can be instrumentally elaborate. ${ }^{5,6}$ In addition, even if pulse spreading could be compensated completely, decreasing the pulse duration below the point where its spectral width exceeds the excitation spectral width of the fluorophore in use decreases excitation efficiency. ${ }^{6}$ This sets a lower bound to the minimal desirable pulse duration, which for typical fluorophores with two-photon excitation spectral widths ${ }^{7,8}$ between 50 and $150 \mathrm{~nm}$ is between 25 and 5 fs, respectively. Increasing the two-photon fluorescence yield by decreasing the repetition rate is limited because Eqn. (2.2) holds true only as long as the excitation probability of a fluorophore per pulse is much smaller than unity. This is due to the fact that the excited-state life-time of most fluorophores (a few nanoseconds) is much longer than the pulse duration ( $\sim 100 \mathrm{fs}$ ) and thus insufficient to relax to the ground state, a prerequisite for the absorption of another pair of photons. Hence, if the excitation probability approaches unity, saturation effects begin to occur. However, even if saturation poses no problem, in view of imaging applications, the excitation pulse repetition rate clearly cannot be lower than the pixel

\footnotetext{
${ }^{\dagger}$ Eqn. (2.2) holds true for the paraxial approximation.
} 
rate of the image acquisition (typically $50 \mathrm{kHz}-2 \mathrm{MHz}$ ), since at least one pulse has to be delivered per pixel.

\subsection{Biological limits and fluorophore characteristics}

The viability of biological specimens during imaging is of paramount importance for any living-tissue imaging technique. Thus, optimization of the two-photon excitation efficiency is also limited (in addition to the physical constraints discussed above) by the degree of damage the specimen can tolerate. In particular, two-photon absorption has been associated with different mechanisms of light-induced damage such as thermal and photooxidative damage. ${ }^{9-}$ ${ }^{11}$ As a rule of thumb, excitation intensity should be set to a level just enough to generate sufficient excitation. However, if peak-intensity related problems persist, increasing the pulse duration or the focal volume (e.g. underfilling the objective's back-aperture) might improve the situation yet reduce the signal to noise ratio. Note that the larger the fluorophore's absorption cross-section and the higher its quantum efficiency, the less the excitation laser intensity required for imaging (and the deeper one can look into tissue). Thus, optimising the two-photon absorption properties of fluorophores helps to increase specimen viability. Efforts to create fluorophores with large two-photon-absorption cross sections have intensified ${ }^{12,13}$ leading to the synthesis of fluorophores with $\delta>1,000 \mathrm{GM}$ (about one to two orders of magnitude larger than those of commonly used fluorescent probes; $\delta_{\mathrm{EGFP}} \sim 100 \mathrm{GM},{ }^{14,15}$ for example).

Although most fluorophores can be excited in two-photon mode at twice the wavelength of their one-photon absorption maximum, two-photon excitation spectra are often significantly different from their one-photon counterparts ${ }^{7}$ due to the different parity selection rules ${ }^{\dagger}$ that apply to one and two-photon transitions. In particular, in a number of fluorophores the twophoton excitation spectrum shows a significant blue shift (i.e. a shift towards higher energy transitions) compared to their one-photon counterpart. ${ }^{8}$ At the same time, no red shift has been reported so far. The reason for this may be that fluorescence emission will only occur for transitions from one-photon allowed excited states which means that, although lower excited states might be accessible via two-photon absorption, those will not be fluorescent. This is

\footnotetext{
${ }^{\dagger}$ For symmetric molecules, for example, quantum parity reverses between one-photon and two-photon excitation processes. Thus, molecular states that are accessible with one-photon excitation may not be accessible in the two-photon case and vice versa.
} 
supported by the fact that fluorescence emission spectra under one- and two-photon excitation are usually identical. ${ }^{7}$ Hence, the same excited state is occupied before the system relaxes to its ground state ${ }^{\ddagger}$. The necessary transition from the two-photon excited state to the one photon excited state is thereby most likely achieved via vibrational coupling allowing the parity forbidden state. ${ }^{8}$ Yet, calculation of two-photon cross sections and quantitative predictions on the basis of known one-photon cross sections are difficult. For choosing the optimum wavelength for two-photon excitation one, therefore, relies heavily on specific measurements of two-photon absorption spectra.

\subsection{Instrumentation}

\subsubsection{General set-up}

Two-photon microscopes are laser-scanning microscopes and their architecture is essentially identical to that found in confocal microscopes. In fact, commercial confocal laser-scanning instruments can easily be converted for two-photon operation, in general requiring only the replacement of optics in the excitation path to adapt to the different excitation wavelengths. ${ }^{1}$ However, one important advantage of two-photon microscopy is that in most situations, outof-focus fluorescence is negligible. Hence, rejection of non-focal fluorescence photons by a confocal pinhole, is rarely desirable. This offers the opportunity for whole-field detection: all fluorescence photons that enter the objective and are passed by the detection filter, are collected. This not only increases the detection efficiency but also allows a simplification of the optical design.

The schematic of a generic two-photon microscopy set-up is shown in Fig. 2.2. The main components are an infrared ultra-short pulse laser as the excitation light source, scan-mirrors and optics, and a fluorescence detector. Images are obtained by raster scanning the focused laser beam across a specimen. Fluorescence is usually collected by the same objective used for focusing of the excitation light, separated by a dichroic mirror and focused onto a detector. The detector signal is then used to build up the image sequentially in a raster pattern.

\footnotetext{
* Assuming the same excited state prior to fluorophore relaxation, the fluorescence quantum efficiency for oneand two-photon excitation can be expected to be equal.
} 


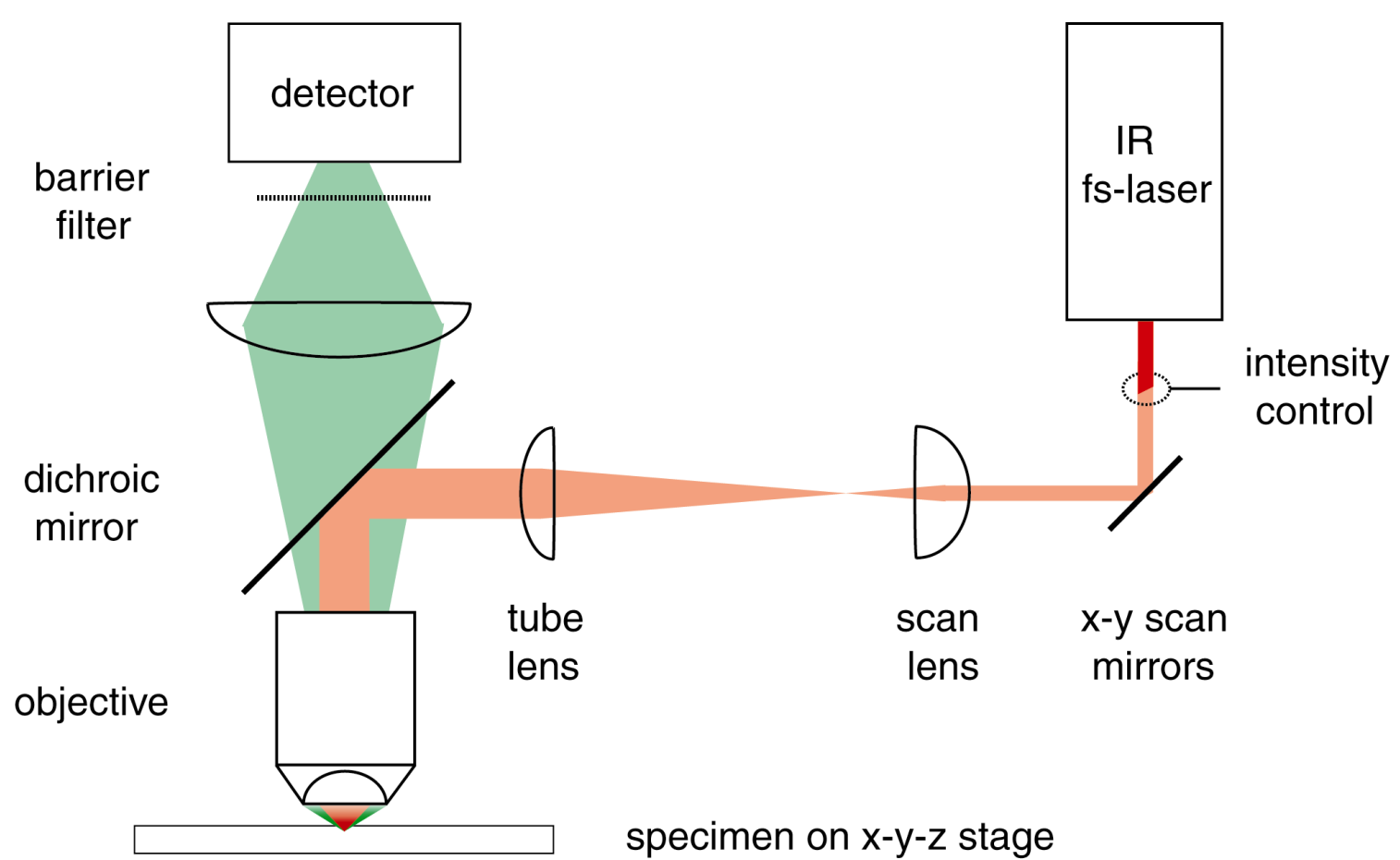

Fig. 2.2 Generic two-photon laser-scanning microscope.

\subsubsection{Light sources}

Excitation light sources that have been used in two-photon microscopy include solid-state lasers such as Cr:LiSAF, Nd:YLF, Nd:glass, and Cr:fosterite, as well as dye- and fiber-based lasers (for a review see Squier et al. ${ }^{16}$ ). The most widely used light source is, however, the Ti:sapphire laser. Due to its high average power capability (about $1 \mathrm{~W}$ ), broad tuning range (700-1100 nm), short pulse duration (less than $100 \mathrm{fs}$ ), as well as reliable and robust operation, it has become the source of choice.

\subsubsection{Scan mirrors and optics}

Optics in the excitation path must, of course, be selected for high throughput in the infrared. For the scan mirrors, protected silver coatings should be used as they offer excellent reflectivity in this wavelength range ${ }^{\dagger}$.

\footnotetext{
${ }^{\dagger}$ dielectric coatings can provide even better performance but are hardly available for the scan mirrors
} 


\subsubsection{Detection system}

With whole-field detection as the preferred fluorescence detection modality in two-photon microscopy, the detection system should ensure the collection of most of the fluorescence photons emerging from the specimen. To achieve this goal, low magnification, high numerical aperture (NA) objectives and high-transmission collection optics should be used. In addition, the detection system must be carefully designed to match the objective's effective angular acceptance which generally requires a dichroic mirror and collecting lens with large clear apertures to be placed as close as possible to the objective. ${ }^{17}$ Rejection of residual excitation light can be achieved by inserting a barrier filter. The large separation of excitation and emission wavelengths (several $100 \mathrm{~nm}$ ) allows for the use of colored glass filters which often provide a better performance than the dielectric filters that are generally required for singlephoton fluorescence microscopy.

Since deep in the tissue most of the collected fluorescence photons will have been scattered before entering the objective, the light cone emerging from the objectives back aperture will be somewhat diffuse. The minimal spot-size to which this light can be focused is therefore no longer diffraction limited. The circle of least confusion can be quite large (several $\mathrm{mm}$ in radius). Thus, large-area detectors (several ten $\mathrm{mm}^{2}$ ) need to be used for efficient detection. In addition, detectors should, of course, feature high quantum efficiency at the emission wavelength, and internal gain to avoid the excess noise introduced by external amplification. Detectors most suitable for two-photon microscopy are photomultiplier tubes (PMT) and avalanche photo diodes (APD). Although APDs are comparatively superior in terms of quantum efficiency $(\sim 80 \%)$, their small sensitive area, low internal gain $\left(\sim 10^{2}\right)$, and excess noise, limits their range of use. In contrast, PMTs are available with large sensitive areas, quantum efficiencies up to $30 \%$, and very high internal gain $\left(>10^{6}\right)$ with no need for further amplification (for an extensive review of detector designs see $\mathrm{Art}^{18}$ ). 


\section{References}

1. W. Denk, J. H. Strickler, and W. W. Webb, "2-Photon Laser Scanning Fluorescence Microscopy," Science 248(4951), 73-76 (1990).

2. M. Göppert-Mayer, "Über Elementarakte mit zwei Quantensprüngen," Annalen der Physik 9, 273-294 (1931).

3. W. Kaiser and C. G. B. Garrett, "2-Photon Excitation in Caf2 - Eu2+," Physical Review Letters 7(6), 229-\& (1961).

4. P. E. Hanninen, E. Soini, and S. W. Hell, "Continuous-Wave Excitation 2-Photon Fluorescence Microscopy," Journal of Microscopy-Oxford 176, 222-225 (1994).

5. M. Muller, J. Squier, R. Wolleschensky, U. Simon, and G. J. Brakenhoff, "Dispersion pre-compensation of 15 femtosecond optical pulses for high-numerical-aperture objectives," Journal of Microscopy-Oxford 191, 141-150 (1998).

6. W. Denk, D. W. Piston, and W. W. Webb, "Two-photon molecular excitation in laserscanning microscopy," in Handbook of Biological Confocal Microscopy, J. B. Pawley, ed. (Plenum Press, New York, 1995), pp. 445-458.

7. C. Xu and W. W. Webb, "Measurement of two-photon excitation cross sections of molecular fluorophores with data from 690 to $1050 \mathrm{~nm}$," Journal of the Optical Society of America B-Optical Physics 13(3), 481-491 (1996).

8. M. A. Albota, C. Xu, and W. W. Webb, "Two-photon fluorescence excitation cross sections of biomolecular probes from 690 to 960 nm," Applied Optics 37(31), 73527356 (1998).

9. H. J. Koester, D. Baur, R. Uhl, and S. W. Hell, "Ca2+ fluorescence imaging with picoand femtosecond two-photon excitation: Signal and photodamage," Biophysical Journal 77(4), 2226-2236 (1999).

10. A. Hopt and E. Neher, "Highly nonlinear photodamage in two-photon fluorescence microscopy," Biophysical Journal 80(4), 2029-2036 (2001).

11. K. Konig, T. W. Becker, P. Fischer, I. Riemann, and K. J. Halbhuber, "Pulse-length dependence of cellular response to intense near-infrared laser pulses in multiphoton microscopes," Optics Letters 24(2), 113-115 (1999).

12. M. Albota, D. Beljonne, J. L. Bredas, J. E. Ehrlich, J. Y. Fu, A. A. Heikal, S. E. Hess, T. Kogej, M. D. Levin, S. R. Marder, D. McCord-Maughon, J. W. Perry, H. Rockel, M. Rumi, C. Subramaniam, W. W. Webb, X. L. Wu, and C. Xu, "Design of organic molecules with large two-photon absorption cross sections," Science 281(5383), 16531656 (1998). 
13. D. R. Larson, W. R. Zipfel, R. M. Williams, S. W. Clark, M. P. Bruchez, F. W. Wise, and W. W. Webb, "Water-soluble quantum dots for multiphoton fluorescence imaging in vivo," Science 300(5624), 1434-1436 (2003).

14. P. Schwille, U. Haupts, S. Maiti, and W. W. Webb, "Molecular dynamics in living cells observed by fluorescence correlation spectroscopy with one- and two-photon excitation," Biophysical Journal 77(4), 2251-2265 (1999).

15. C. Xu, "Two-photon Cross Sections of Indicators," in Imaging Neurons, R. Yuste, F. Lanni, and A. Konnerth, eds. (Cold Spring Harbor Laboratory Press, Cold Spring Harbor, 2000).

16. J. Squier and M. Muller, "High resolution nonlinear microscopy: A review of sources and methods for achieving optimal imaging," Review of Scientific Instruments 72(7), 2855-2867 (2001).

17. M. Oheim, E. Beaurepaire, E. Chaigneau, J. Mertz, and S. Charpak, "Two-photon microscopy in brain tissue: parameters influencing the imaging depth," Journal of Neuroscience Methods 111(1), 29-37 (2001).

18. J. Art, "Photon Detectors for confocal Microscopy," in Handbook of Biological Confocal Microscopy, J. B. Pawley, ed. (Plenum Press, New York, 1995). 


\section{Imaging depth in two-photon fluorescence microscopy}

\subsection{Introduction}

One of the main advantages of two-photon microscopy compared to one-photon imaging techniques is that it can provide images from deep within scattering tissue owing to two unique properties. First, the inherent sectioning capability allows very efficient fluorescence collection because virtually all of the generated fluorescence constitutes high-resolution information which is not lost upon subsequent scattering since its origin is known to be the focal volume. Second, scattering is reduces as the wavelength increases, which means that the NIR light typically used in two-photon microscopy penetrates further into the tissue than the visible light (of roughly half that wavelength) used in one-photon excitation. Both of these two properties contribute to the two-photon microscope's ability to provide high resolution images in living tissue at depths that have hitherto not been accessible.

Given the wide range of optical properties of tissue, that depend on cellular content, vascularisation etc. it is difficult to provide definite numbers for the maximum penetration depth in tissue. Apart from these difficulties the term "maximum imaging depth" itself seems to be quite vague because different definitions are conceivable. Certainly no meaningful imaging is possible beyond the point at which the focal fluorescence signal falls below the photon shot-noise level. While the point at which this occurs depends on imaging parameters such as laser power, staining strength, detection efficiency, pixel dwell time, etc. it can be defined by requiring a certain signal-to-noise ratio $(\mathrm{S} / \mathrm{N})$. One way of doing this is to request a minimum average focal excitation power $\left\langle P_{\min }\right\rangle=\langle P\rangle e^{-z_{\max } / l_{s}}$ corresponding to a minimum number of photon pairs $\left(n_{\min }\right)$ absorbed per fluorophore and per unit time. Using Eqn. (2.2), the maximum imaging depth is then given by

$$
z_{\max }=l_{s} \ln \left[\sqrt{\frac{\delta}{n_{\min } \tau f}} \frac{\pi(N A)^{2}}{h c \lambda}\langle P\rangle\right]
$$


which depends linearly on the scattering mean-free-path length $l_{s}$ and logarithmically on excitation power $P$ and duty cycle $(\tau f)$. Hence a large increase in excitation power or decrease in duty cycle is necessary to achieve a significant increase in the maximum imaging depth. Parts of this chapter have been published elsewhere. ${ }^{1,2}$

\subsection{Current limits and strategies for improving the imaging depth}

The lasers commonly used as the excitation source for two-photon microscopy (mainly modelocked Ti: $\mathrm{Al}_{2} \mathrm{O}_{3}$ oscillators providing $100 \mathrm{fs}$ pulses at a repetition rate of $80 \mathrm{MHz}$ ) are limited to an average output power of, currently, about $1 \mathrm{~W}$. This allows imaging to depths of about 2-3 scattering-mean-free-path lengths. ${ }^{3,4}$ Efforts to increase the imaging depth have since concentrated on improving the excitation and collection efficiency.

Investigations on the role of the angular and area acceptance of the detection optics for the imaging depth has revealed the importance of using high numerical aperture and field-of-view lenses. ${ }^{5,}{ }^{6}$ An increase in imaging depth of about $100 \mu \mathrm{m}$ in brain tissue has been predicted when using a 20x/0.95NA objective (Olympus) instead of a 'standard' 60x lens. ${ }^{5}$

Owing to the non-linear dependence of two-photon fluorescence on excitation intensity, the excitation efficiency can be increased by decreasing the laser duty cycle $(\tau f)$, lowering either the repetition rate or the pulse duration (within the limits discussed above). Pulse widths as short as 15 fs have been demonstrated in the focus of a high numerical aperture objective ${ }^{7}-$ corresponding to a 7-fold increase in excitation efficiency compared to the 'standard' $100 \mathrm{fs}$ laser. However, much larger increases in excitation efficiency and thus depth penetration can be expected from lowering the laser repetition rate, ${ }^{8}$ which can be achieved by increasing the cavity length of the oscillator (lowest repetition rate achieved so $\operatorname{far}^{9}: 4 \mathrm{MHz}$ or by cavity dumping (with repetition rates up to ${ }^{10} 950 \mathrm{kHz}$ ). Both methods result, however, in a significant decrease in average power. This can be avoided by the use of a regenerative amplifier, for which repetition rates of up to $400 \mathrm{kHz}$ have been demonstrated. ${ }^{11}$

Considering a typical pixel-rate between $50 \mathrm{kHz}$ and several $\mathrm{MHz}$ as lower bound for the pulse repetition frequency since at least one laser pulse must be delivered per image pixel, an up to 2000-fold increase in excitation efficiency compared to a standard $80 \mathrm{MHz}$ oscillator can be achieved ${ }^{\dagger}$. Using Eqn. 2.3, this translates in an increase in penetration depth by up to 3.8 scattering mean-free-path lengths.

\footnotetext{
${ }^{\dagger}$ Assuming the same average power is provided by the oscillator and amplifier.
} 
Some words of caution regarding the application of high energy pulses for deep imaging of biological specimens are needed: Note, that regardless any improvements in excitation power and efficiency that the imaging depth in two-photon microscopy cannot be increased indefinitely but is limited by the maximum power the specimen can tolerate and more fundamentally by the onset of substantial generation of out-of-focus fluorescence near the top of the sample ${ }^{12}$ because for very thick absorbing or scattering samples the assumption that two-photon fluorescence is largely confined to the focal region is no longer true. This can be explained by the fact that in order to maintain constant signal strength the incident laser power needs to be increased exponentially with depth. For large depths, this exponential increase in power begins to dominate the decrease of excitation efficiency caused by the increase in the beams cross section (scaling quadratically with depth), eventually generating two-photon fluorescence comparable to or exceeding that produced in the focal volume.

\subsection{Regenerative amplification and its applicability for two-photon}

\section{microscopy}

As described in the previous chapter, mode-locked $\mathrm{Ti}: \mathrm{Al}_{2} \mathrm{O}_{3}$ oscillators commonly used as the excitation source in two-photon microscopy typically generate 100 fs pulses with repetition rates of about $80 \mathrm{MHz}$ at a mean output power of up to about $1 \mathrm{~W}$. Corresponding pulse energies are on the order of several nJ. The need for higher pulse energies has triggered the development a number of amplifier configurations ${ }^{13}$ that differ in repetition rate and pulseenergy gain that can be achieved. Both parameters, however, cannot be chosen independently of one another. Instead, the product of pulse energy and repetition rate (the average power) is usually constant and about as large as the output power of the oscillator. The basic principle common to all amplifiers is based on sending the pulses to be increased in energy through a medium that contains a population inversion which provides gain via stimulated emission. On a femtosecond timescale, however, amplification becomes a complex issue. An amplifier design not only has to maintain a short pulse duration but also has to circumvent the problems associated with the extremely high intensities of amplified femtosecond pulses. A popular way of doing this is to stretch the pulse duration to the ps scale prior to or during amplification (chirped pulse amplification) requiring recompression of the pulse after 
amplification. ${ }^{14}$ The most efficient method to transfer energy to a fs pulse from an amplifier with Ti: $\mathrm{Al}_{2} \mathrm{O}_{3}$ as the gain medium, is regenerative amplification. ${ }^{15}$

The concept of regenerative amplification is illustrated in Fig. 3.1. A single pulse (seed) from the fs oscillator is passed through a optical isolator and injected into the amplifier cavity by a acousto-optical cavity dumper (CD). The pulse circulates in the cavity and is amplified upon each passage though the gain medium. After a number of round trips (depending on the energy storage time of the gain medium and the time needed to reach pulse-energy saturation), the pulse is ejected by the cavity dumper, returns through the optical isolator and is separated from the input beam path by a polariser.

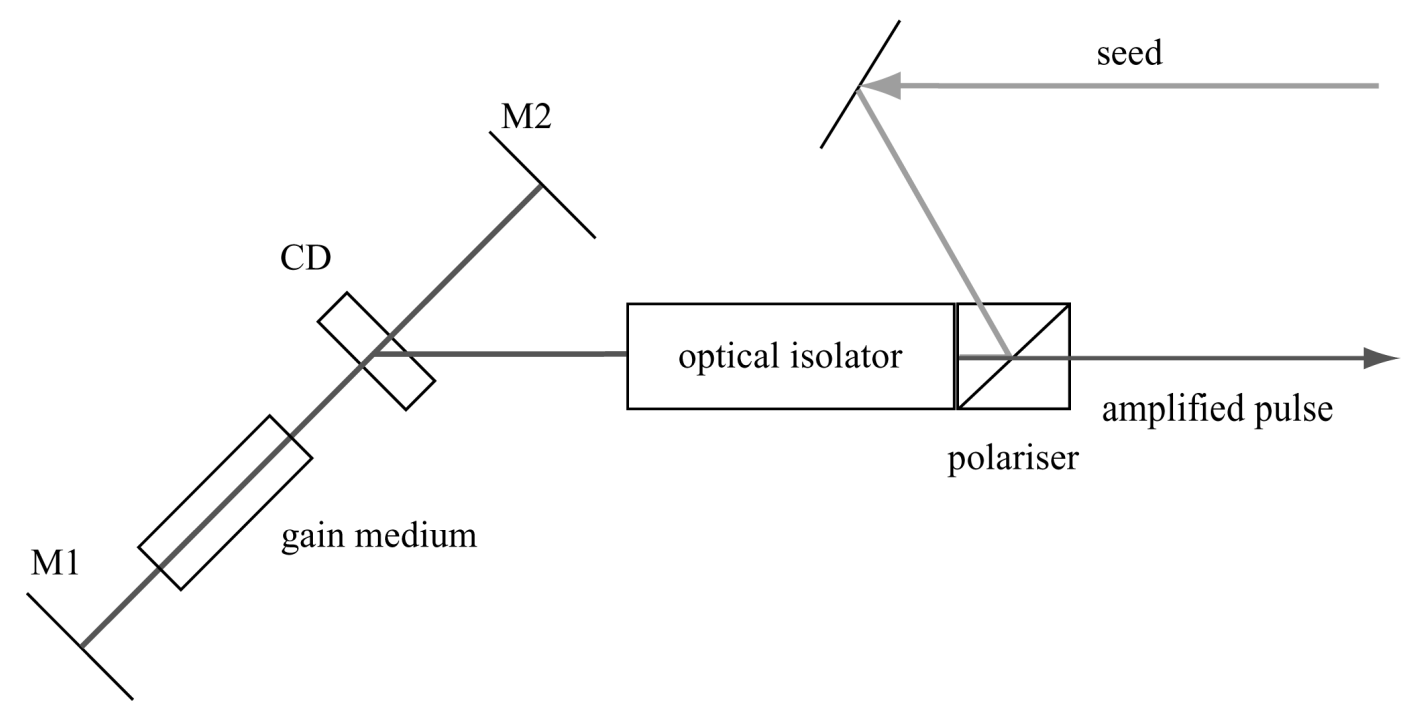

Fig. 3.1 Principle of regenerative amplification.

$\mathrm{Ti}: \mathrm{Al}_{2} \mathrm{O}_{3}$ regenerative amplifiers with high enough repetition rates suitable for imaging have been developed[Norris, 1992 \#68]] and are available commercially, they lack much of the broad tuneability that has been in large part responsible for the popularity of $\mathrm{Ti}_{\mathrm{Al}} \mathrm{Al}_{2} \mathrm{O}_{3}$ oscillators. Although the tuneability of lasers is in general mainly limited by the bandwidth of the gain medium (about $400 \mathrm{~nm}$ for $\mathrm{Ti}: \mathrm{Al}_{2} \mathrm{O}_{3}$ ), in practice amplified spontaneous emission (ASE) limits it to about $\pm 50 \mathrm{~nm}$ around the peak-gain wavelength of about $800 \mathrm{~nm}$ ). ASE is a result of the pump duration being several orders of magnitude longer than the pulse to be amplified. Although lasing in the amplifier cavity is prevented before a pulse is injected, during the amplification of the pulse spontaneous emission is amplified thereby reducing gain and the signal (pulse) to background (ASE) ratio and even cause continuous wave (CW) operation. This can become severe for operation further away from the peak gain wavelength, 
due to the reduced gain for the pulse amplification and result in unstable operation. To suppress ASE the cavity losses for undesired wavelengths have to be increased, for example, by the introduction of wavelength selective elements such as a birefringent filter, which, however, carries the cost of reduced bandwidth. A better, though less versatile, alternative is the introduction of cavity mirrors that do not reflect ASE which has its peak at $800 \mathrm{~nm}$.

Another important point to consider when using a regenerative amplifier as the excitation source in two-photon fluorescence microscopy is the low repetition rate. With only one or at most a small number of pulses/pixel, synchronisation becomes essential since the variation in pulse number per pixel (by 1 pulse without synchronisation) is fractionally large. This is very different from using an ' $80 \mathrm{MHz}$ ' oscillator, where the number of pulses per pixel is between 30 and 2000.

\subsection{Methods and results}

In order to test whether an increase in the two-photon imaging depth of the order of several scattering mean-free-path lengths can indeed be achieved by repetition rate reduction we have imaged a tissue-equivalent phantom, stained vasculature in vivo, and green fluorescent protein (GFP) labelled neurons in vivo using a regenerative amplifier as the excitation source in a two-photon microscope.

\subsubsection{Experimental set-up}

Our set-up (see Fig. 3.2) consists of a regenerative amplifier (RegA 9000, pumped by a 10W Verdi, seeded by a 5W Verdi-pumped Mira 900-F; all from Coherent) coupled into a custombuilt microscope head controlled by a modified micro-manipulator (MP-285, model 3Z, Sutter Instrument Company). Fluorescence is separated with a dichroic mirror and barrier filter (BG38, Schott) and detected with a photomultiplier (R3896, Hamamatsu). The excitation intensity can be adjusted using a galvo-scanner mounted crystalline quartz plate based on the principle of Berek's compensator. ${ }^{16}$ 


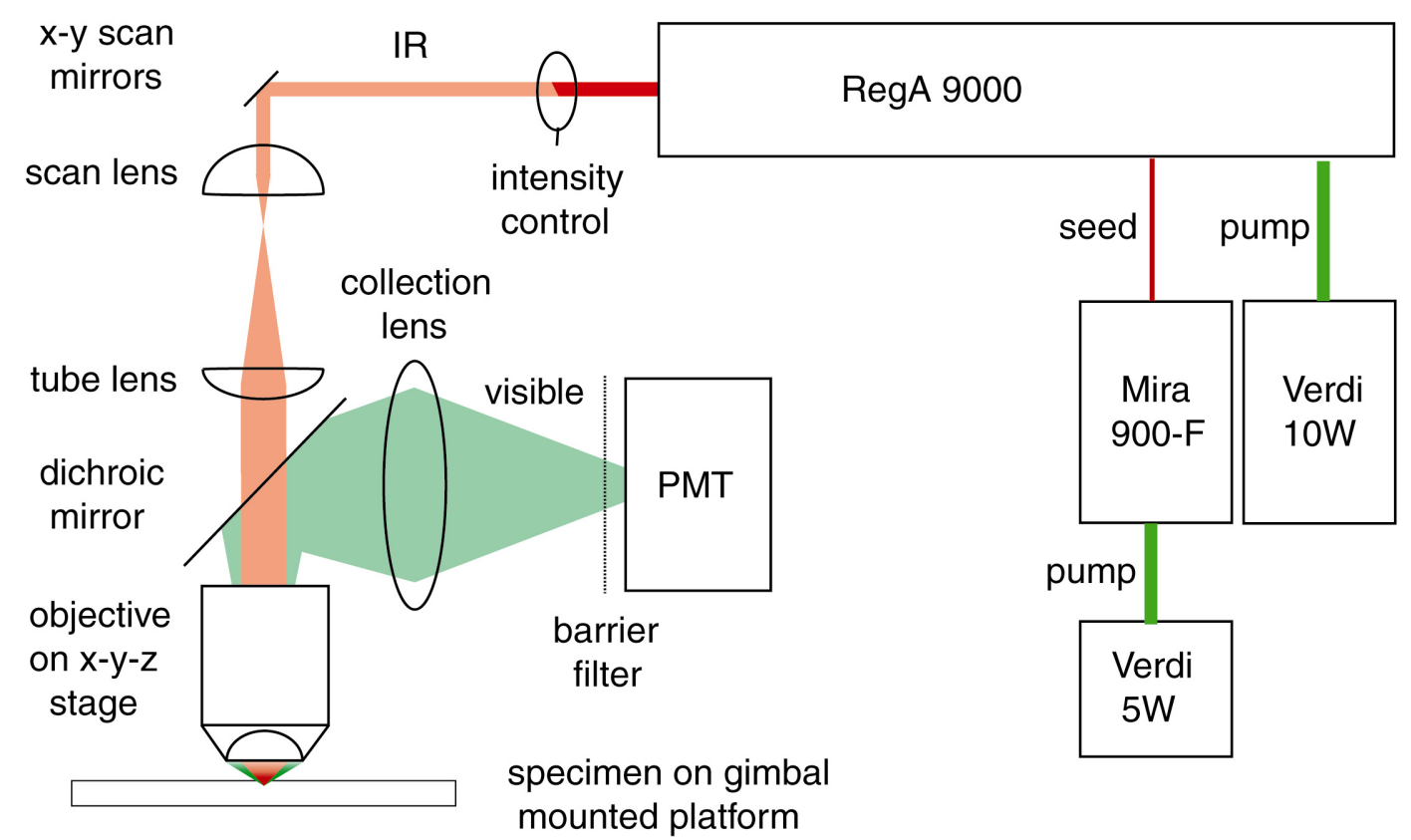

Fig. 3.2 Experimental set-up of the two-photon fluorescence microscope with a regenerative amplifier as the excitation source.

For efficient excitation of green fluorescent protein (GFP), the regenerative amplifier has been modified for operation at wavelengths around $910 \mathrm{~nm}$ using a selectively reflecting mirror (custom design, MH 009814, CVI) which was inserted in exchange of the flat cavity end mirror (M6) (see Fig. 3.3). The spectral characteristics of the long pass filter are shown in Fig. 3.4 .

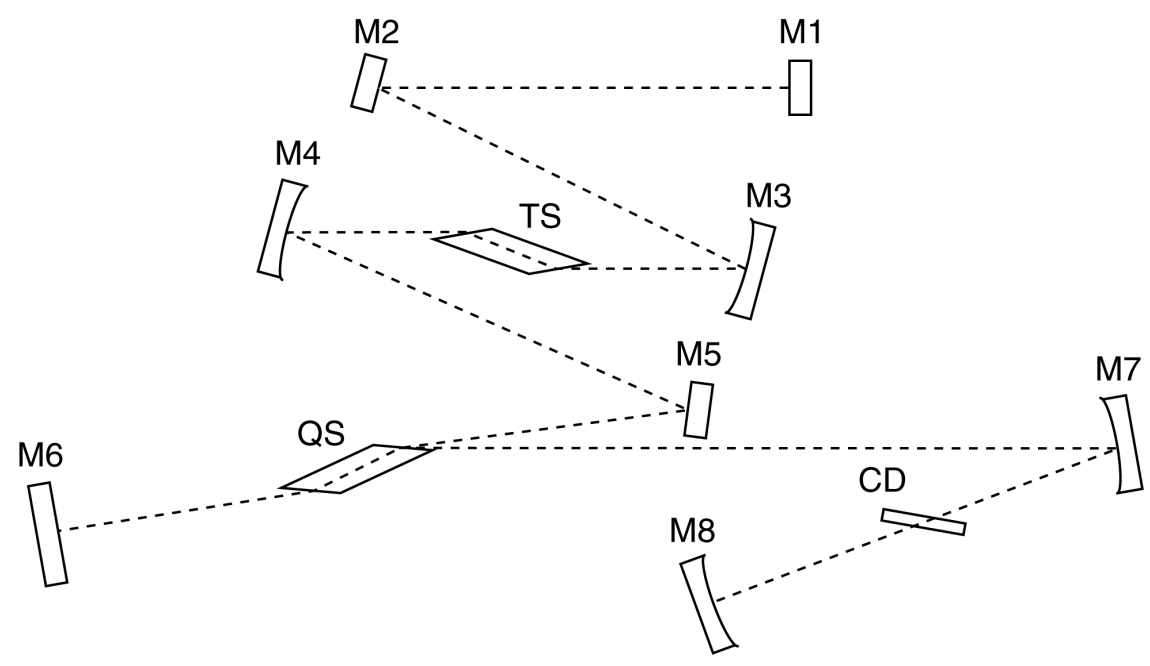

Fig. 3.3 Sketch of the optical cavity layout of the regenerative amplifier (RegA 9000, Coherent) with cavity mirrors (M1-M8), Ti:Sapphire crystal (TS), Q-switch (QS) and cavity dumper (CD). For a complete layout of the amplifier head refer to its Operator's Manual (RegA Model 9000 Laser, Coherent, Santa Clara, CA 95054). 


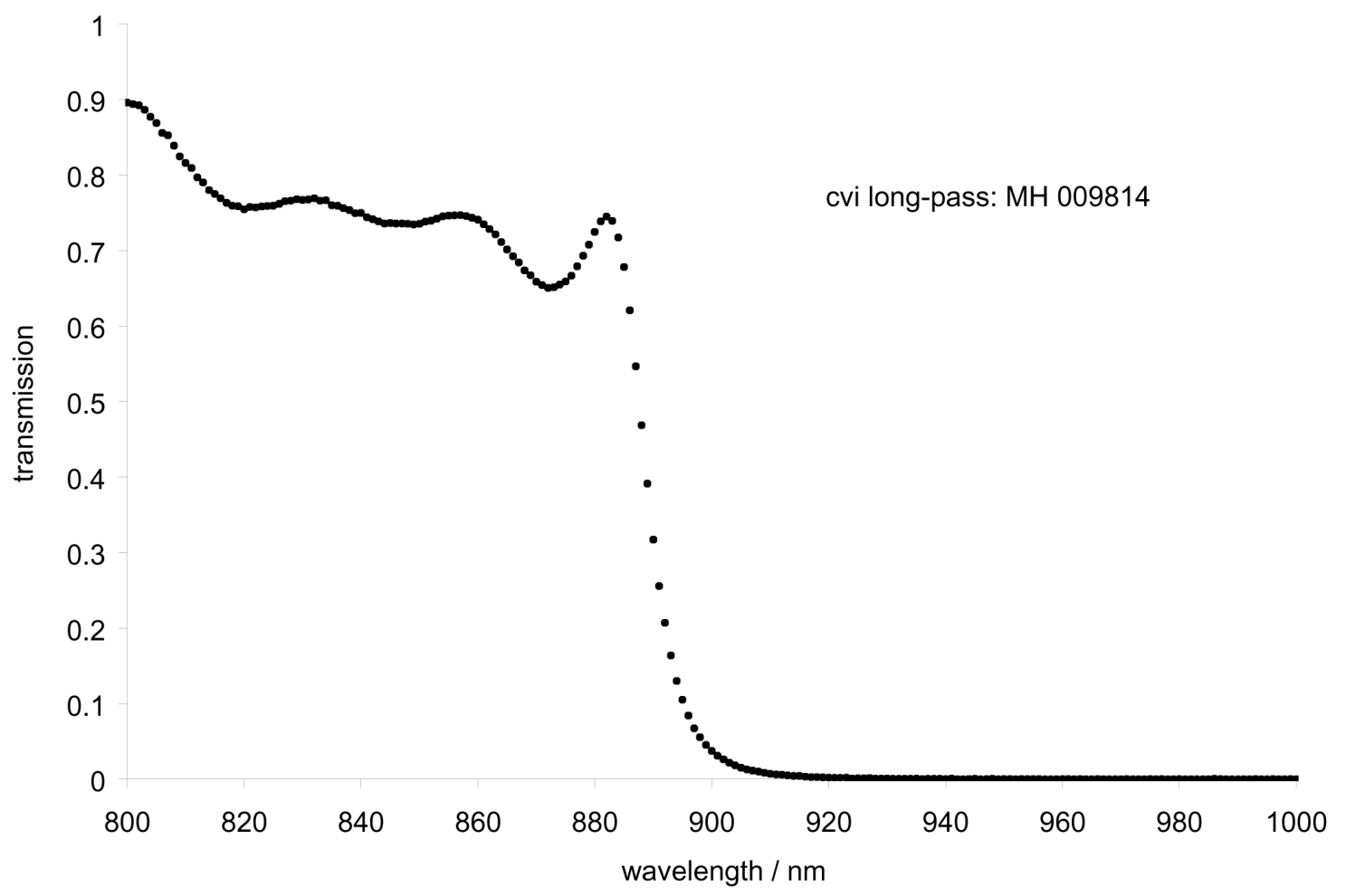

Fig. 3.4 Transmission characteristics of special long wavelength reflector mirror used (MH 009814, CVI; $_{\max }$ @ $950 \mathrm{~nm}, \theta=0^{\circ} ; \mathrm{T}=50 \% @ 888 \mathrm{~nm}$ ) recorded with a spectrophotometer (Cary 500, Varian Inc., resolution: 1 $\mathrm{nm}$, integration time: $0.1 \mathrm{~s})$.

At a repetition rate of $200 \mathrm{kHz}$ the RegA provides an average power of $600 \mathrm{~mW}$ at a centre wavelength $\lambda_{0}=909 \mathrm{~nm}$. Stable operation of the amplifier was verified by recording the spectrum and pulse-duration using a laser spectrometer (WaveScan, APE GmbH) and an autocorrelator (PulseScope, APE GmbH), respectively (see Fig. 3.5). The measured spectral and temporal widths (FWHM) are $\Delta \lambda=10.78 \pm 0.04 \mathrm{~nm}$ and $\tau=158.12 \pm 0.47 \mathrm{fs}$ (assuming a $\operatorname{sech}^{2}(t)$ pulse shape to correct the autocorrelation width of $\tau_{\text {ac }}=244.02 \pm 0.73$ fs i.e. $\tau=$ $\left.0.648 \tau_{\mathrm{ac}}\right)$ giving a time-bandwidth product of $\tau \Delta f=\tau c \Delta \lambda / \lambda_{0}=0.619 \pm 0.005$ which is roughly two times larger than the expected time-bandwidth limit (0.315) and most likely due to uncompensated higher-order phase dispersion (also apparent by the cantilevered wings of the autocorrelation trace ${ }^{17}$ in Fig. 3.5b). 


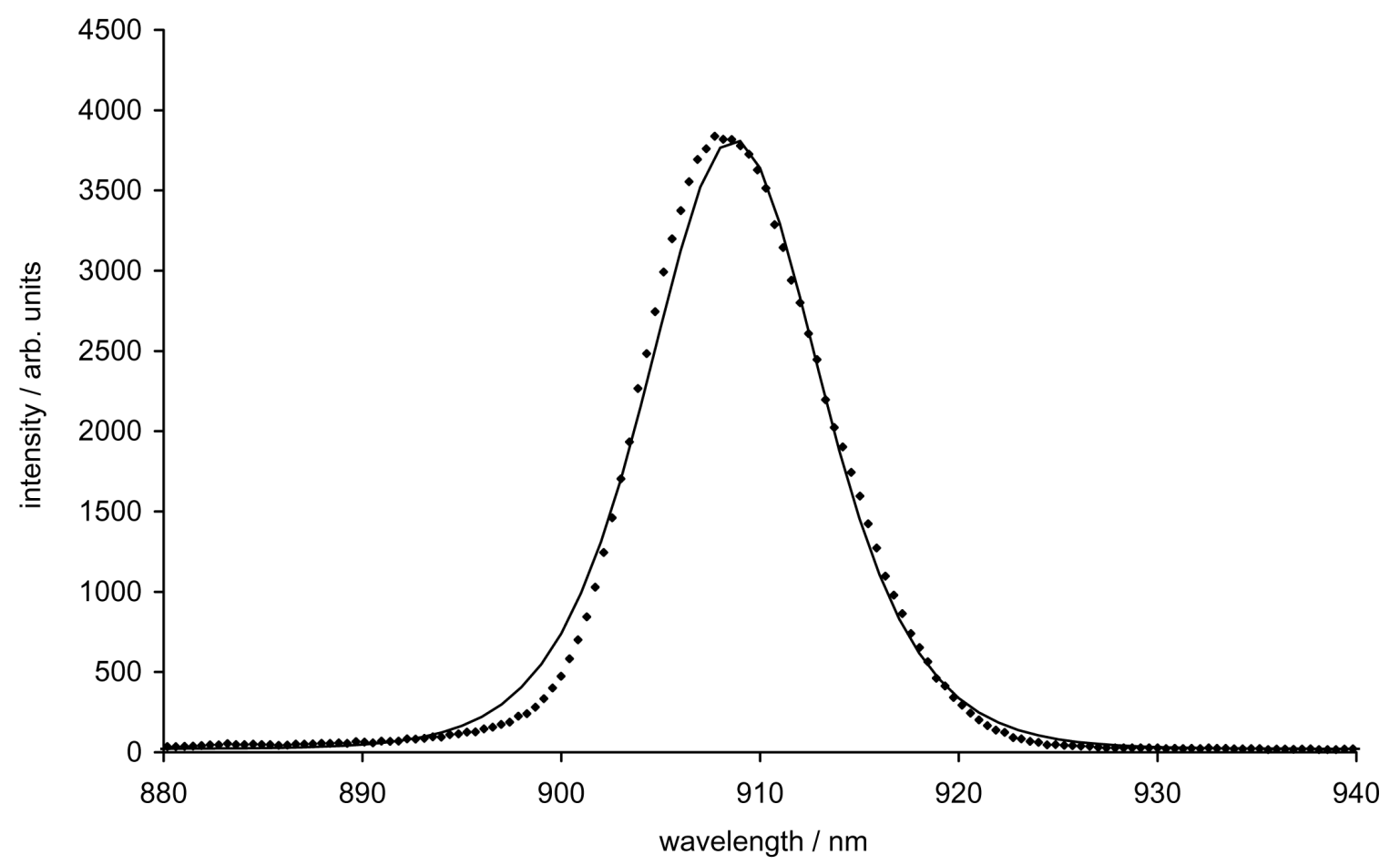

Fig. 3.5a Spectrum of RegA with fitted $\operatorname{sech}^{2}\left(\mathrm{R}^{2}=0.996\right), \mathrm{FWHM}=10.78 \pm 0.04 \mathrm{~nm}, \lambda_{0}=908.70 \pm$ $0.02 \mathrm{~nm}$. The asymmetry of the spectrum is due to the long-pass filter used to eliminate ASE (see text) which leads to a steepening of the spectrum's low wavelength edge.

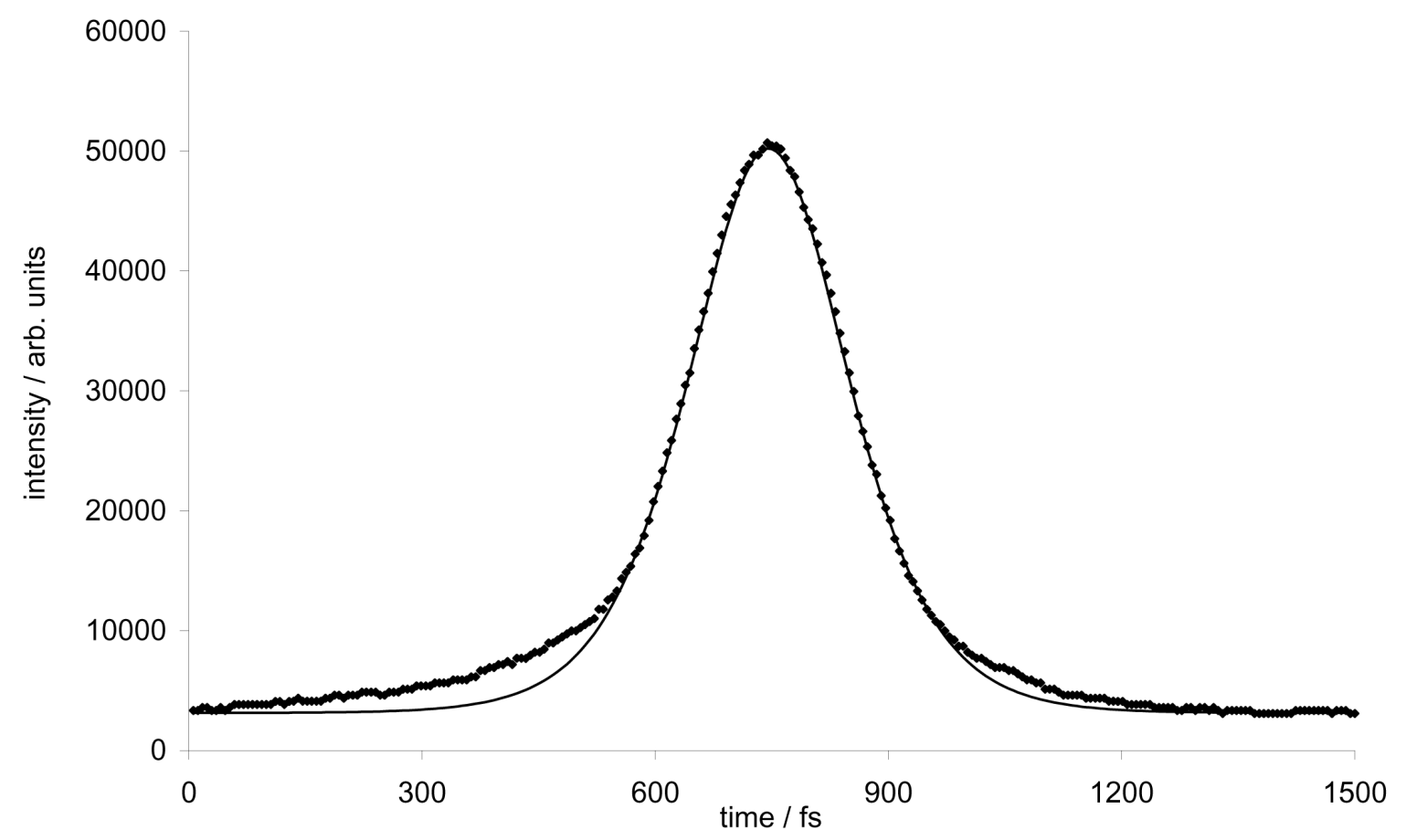

Fig. 3.5b Autocorrelation trace of amplified output pulses. Superimposed is the fitted $\operatorname{sech}^{2}$ function $\left(\mathrm{R}^{2}\right.$ $=0.989), \mathrm{FWHM}=244.02 \pm 0.73 \mathrm{fs}$. Note the cantilevered flanks, indicating the presence of third and higher order GVD. 
The RegA 9000 was synchronised to the pixel clock of the image-acquisition system to ensure the same number of pulses for each pixel integration period. Synchronisation was achieved using a D flip-flop based circuit receiving a $\sim 38 \mathrm{MHz}$ TTL input from the RegA master clock (derived from the $\sim 76 \mathrm{MHz}$ photodiode output of the MIRA oscillator i.e. the oscillator pulse repetition frequency), a TTL input from the pixel clock of the image acquisition system and providing a TTL repetition-rate-trigger output to the RegA electronics (see Fig. 3.6).

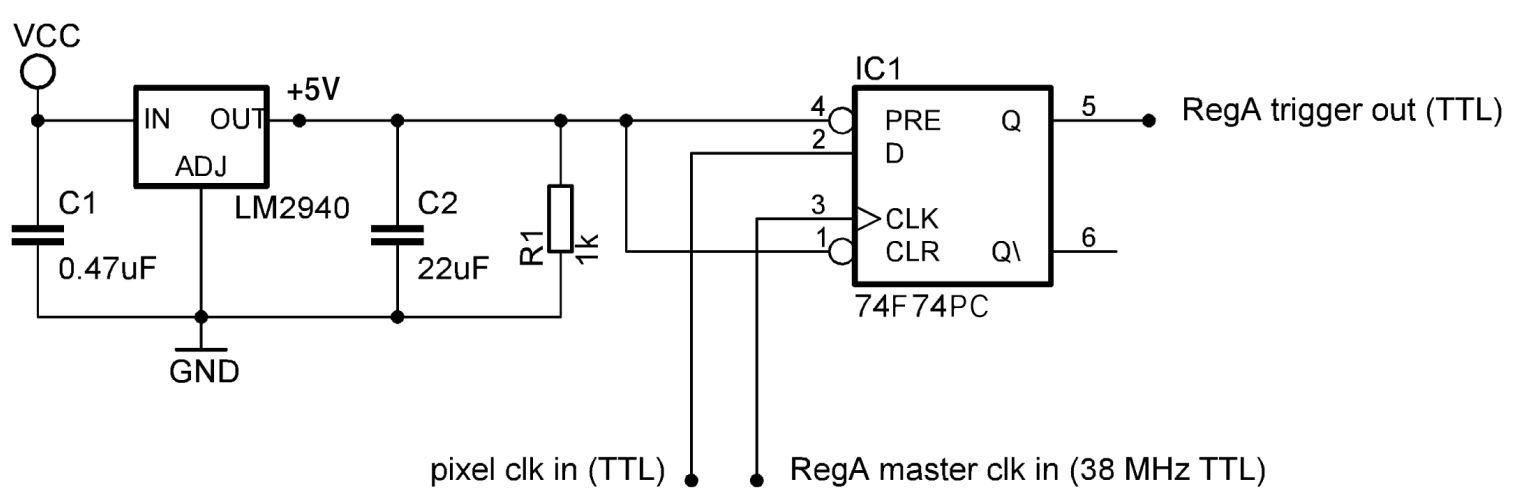

Fig. 3.6 Synchronization circuit based on a flip-flop (74F74PC) and a low dropout voltage regulator (LM2940).

The pulse width at the focus of the objective used (Nikon Fluor DIC M 40x/0.80W) was measured using an autocorrelator (PulseScope, APE GmbH) after first recollimating the beam using a reflecting objective (36x/0.5, Ealing/Coherent). The minimal pulse width achievable by adjusting the post-amplification grating compressor (the original grating compressor of the RegA has been placed outside of the enclosing to provide easier access) has been found to differ only slightly from the initial value indicating the order of GVD accumulated by passing through the microscope optics to be mainly quadratic.

\subsubsection{Tissue phantom}

In a first step we tested the set-up by imaging fluorescently labelled beads in a tissue phantom. In order to design a phantom that mimics the properties of biological tissue relevant to two-photon microscopy, one has to consider the processes governing the propagation of

\footnotetext{
${ }^{\dagger}$ A digital logic device that stores the status of one of its two inputs (D) whenever its second input (CLK) makes a certain transition (low to high or high to low). The state of its output (Q), is given by the currently stored value. For more information see for example Horowitz et al. ${ }^{18}$.
} 
light which are refraction, scattering, and absorption. These processes are quantified by the index of refraction $(n)$ determining the speed of light in a medium, the scattering cross section $\left(\sigma_{\mathrm{s}}\right)$, the differential scattering cross section $(d \sigma / d \Omega)$ i.e. the angular distribution of scattered light and the absorption cross section $\left(\sigma_{\mathrm{a}}\right)$.

\section{Refractive index}

Because of the heterogeneity of most biological tissues, the refractive index is a complex function of space and is difficult to measure. However, in most applications, it is sufficient to know the effective refractive index of bulk tissue, which can be approximated by the volumeweighted average of the refraction indices of its constituents. ${ }^{19}$ In soft tissues this ranges from 1.33 for water which makes up the main portion of most biological tissues to about 1.6 for melanin in the epidermal layer of skin. ${ }^{20}$ However, the effective index of refraction for most tissues is not so different from that of water i.e. $<1.40$. For a comprehensive list of refractive indices for tissues and tissue constituents see for example Mobley et al. ${ }^{20}$ and references therein.

\section{Scattering parameters}

Scattering occurs where the refractive index changes e.g. due to localised particles with refractive indices different from their surroundings or density gradients. In biological tissues, the most important scattering centers are cells and their organelles ranging in size from $8 \mathrm{~nm}$ (actin filaments) to several tens of microns for the largest cell bodies. ${ }^{21}$ For particles much smaller than the wavelength of the incident light, the intensity pattern of the scattered light is essentially that of an electric dipole ${ }^{22}$ (Rayleigh scattering). However, the majority of scatterers are of a size comparable to the wavelength and exhibit a highly anisotropic forward scattering, which can be described by the theory of Mie, ${ }^{23}$ includes Rayleigh scattering as a limiting case. Though derived for the scattering of a single sphere, Mie's theory also applies to any number and size of spheres, provided they are distributed randomly and separated from each other by distances large compared to the wavelength of the incident light. ${ }^{24}$ Under these circumstances coherent phase relationships are random and interference between the light scattered by the different spheres is thus negligible. With this assumption, which is valid for most imaging applications, the total scattering power is equal to the power scattered by a 
single sphere of a certain size multiplied with the total number of such spheres and summed over all sizes present in the sample. ${ }^{25}$ The power scattered by a single sphere is given by the product of incident intensity and the sphere's scattering cross section $P_{\text {scat }}=\sigma_{s} I_{0}$. The attenuation of unscattered light propagating through a non-absorbing medium containing a large number of randomly but homogeneous distributed scatterers with different scattering cross sections can then be characterised by a scattering coefficient i.e. the cross sectional area for scattering per unit volume

$$
\mu_{s}=\sum_{i}\left(\rho \sigma_{s}\right)_{i}
$$

where $\rho$ is the number density of spheres of equal scattering cross section. Often a more intuitive measure is the scattering mean-free-path length which is the average distance travelled by a photon between successive scattering events and is given by the reciprocal scattering coefficient $l_{s}=\mu_{s}^{-1}$.

Since the majority of scatterers encountered in biological tissues deviates more or less from a spherical shape, the application of Mie's theory to biological tissues seems questionable. On the other hand, because of the large number of different shapes and irregularities of scatterers encountered in biological tissues and since their exact form, size and composition is usually unknown, using a single model shape to mimic tissue scatterers accurately is bound to fail. Methods based on the statistical irregularities of the refractive index ${ }^{26}$ may thus be more adequate. However, especially in the context of modelling light propagation over distances much longer than one scattering mean-free-path length, assuming particles of spherical symmetry, is a rough but adequate approximation since multiple scattering will average out differences due to shape and orientation. Nevertheless, in tissues with only a small variation in the shape of scatterers such as muscle, the use of non-spherical model shapes would probably improve the modelling accuracy (for a selection of solutions of the scattering problem for non-spherical particles see for example van de $\mathrm{Hulst}^{25}$ and references therein).

The angular distribution of light scattered by a single particle is described by the differential scattering cross section ${ }^{20}$

$$
\frac{d \sigma_{s}}{d \Omega}\left(\mathbf{s}, \mathbf{s}^{\prime}\right)=\frac{P_{s c a t}}{I_{0}}
$$


where $\mathbf{s}$ and $\mathbf{s}$, are unit vectors with the directions of the incident and scattered light respectively.

Eqn. 3.3 is also valid for a collection of particles using the same argument as above provided that multiple scattering is negligible, which requires the use of samples with a thickness much smaller than a scattering mean-free-path length.

With the assumption that the scatterers possess spherical symmetry, the differential scattering cross section depends only on the relative orientation between incident and scattered light and can thus be written as a function of the cosine of the angle $\theta$ between the direction of the incident light $\mathbf{s}$ and the scattered light $\mathbf{s}^{, 20}$

$$
\frac{d \sigma_{s}}{d \Omega}\left(\mathbf{s}, \mathbf{s}^{\prime}\right)=\frac{d \sigma_{s}}{d \Omega}\left(\mathbf{s} \cdot \mathbf{s}^{\prime}\right)=\frac{d \sigma_{s}}{d \Omega}(\cos (\theta))
$$

Equation. 3.4 is often normalised to the albedo, which is the fraction of the total cross section that is due to scattering and is given by the ratio of the scattering cross section to the total extinction cross section:

$$
\gamma=\frac{\sigma_{s}}{\sigma_{s}+\sigma_{a}}
$$

This yields the angular scattering distribution or phase function

$$
p(\cos (\theta))=\gamma \frac{4 \pi}{\sigma_{s}} \frac{d \sigma_{s}}{d \Omega}(\cos (\theta))
$$

Another important parameter is the anisotropy factor $g$ which is defined as the average cosine over the phase function ${ }^{27}$

$$
g=\langle x\rangle=\frac{2 \pi}{\gamma} \int_{-1}^{1} p(x) x d x ; \quad x=\cos (\theta)
$$

It is a measure of total forward momentum carried by the scattered light ranging from 1 to -1 corresponding to pure forward $(g=1)$ and backward $(g=-1)$ scattering behaviour. For $g=0$, light is scattered isotropically. 
As mentioned above, most biological tissues exhibit a phase function that is strongly peaked in the forward direction, which is typical for scatterers comparable to the wavelength. For the reasons discussed above, the calculation of an exact scattering phase function is difficult but a reasonable approximation can be made by using phase functions that resemble those computed on the basis of Mie's theory for particles whose size is comparable to the wavelength of the incident light. A phase function commonly used in biological tissue optics $^{28-30}$ is the Henyey-Greenstein phase function ${ }^{31}$

$$
p_{H G}(x)=\frac{\gamma}{4 \pi} \frac{1-g^{2}}{\left(1+g^{2}-2 g x\right)^{3 / 2}} ; \quad x=\cos (\theta)
$$

This phase function is convenient for many applications because of its analytic form, which has a simple expansion in Legendre polynomials, and its parameterisation by the anisotropy factor $g$. A polar plot of the Henyey-Greenstein phase function for an anisotropy factor of 0.9 (strong forward scattering) is shown in Fig. 3.7.

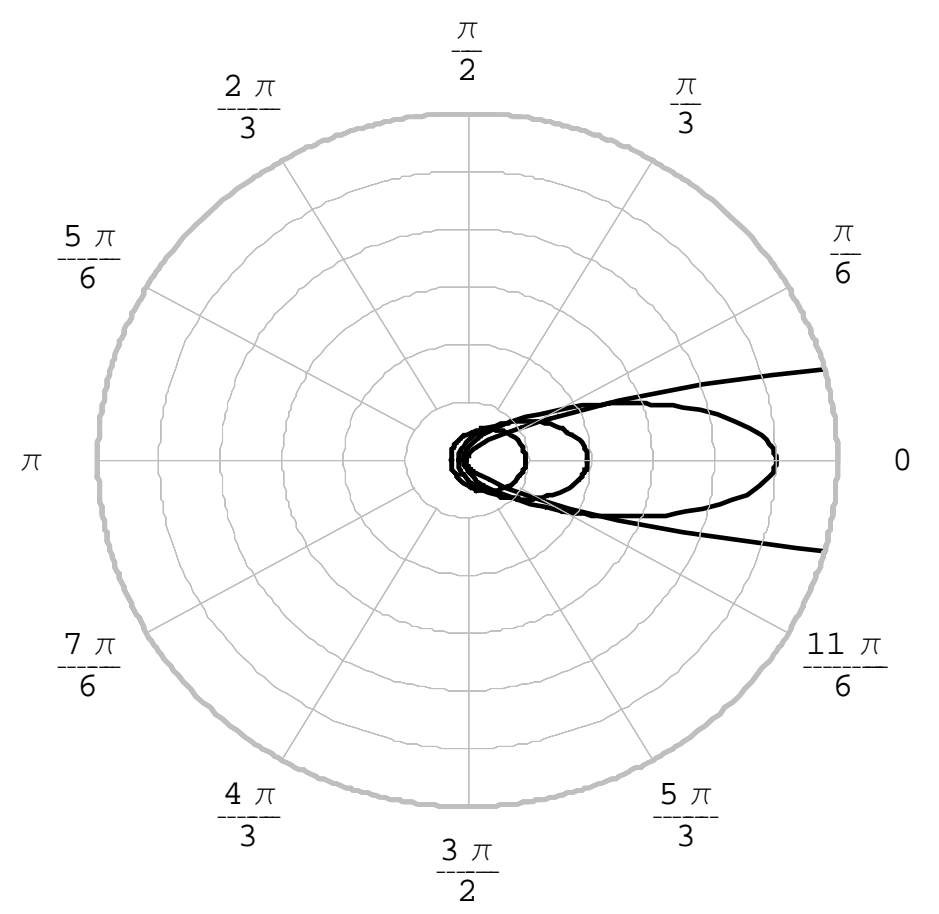

Fig. 3.7 Polar diagram of the Henyey-Greenstein phase function (Eqn. 3.7) for $\gamma=1$ and different anisotropy factors $g=0.2,0.4,0.6,0.8$. 
While the scattering mean-free-path length of biological tissues varies widely with tissue type and wavelength - spanning almost three orders of magnitude from more than $1 \mathrm{~mm}$ in fatty tissue of the female breast at wavelengths in the near infrared ${ }^{32}$ to a few microns in skin at wavelengths near the $\mathrm{UV}^{33}$ - the variation in the anisotropy factor is quite small, ranging between 0.8 and 1 (see Mobley et al. ${ }^{20}$ and references therein). What is also apparent is that the increase of the scattering mean-free-path length with wavelength, is also true for the anisotropy factor though to a smaller degree. The change in scattering mean-free-path length can be quite large. In the epidermis for example, the scattering mean-free-path length changes by a factor of about 5 between 250 and $800 \mathrm{~nm} .{ }^{33}$ This factor is, however, still about 20 times smaller compared to what is expected in the case of pure Rayleigh scattering where the scattering mean-free-path length scales as the forth power of the wavelength. ${ }^{22}$

\section{Absorption cross section}

Similar to the scattering cross section the absorption cross section can be defined as the absorbed power divided by the incident light intensity

$$
\sigma_{a}=\frac{P_{a b s}}{I_{0}}
$$

In the same manner one obtains the absorption coefficient and mean-free-path length

$$
\begin{gathered}
\mu_{a}=\sum_{i}\left(\rho \sigma_{a}\right)_{i} \\
l_{a}=\mu_{a}^{-1} .
\end{gathered}
$$

Based on the characteristics of the major tissue chromophores (see Fig. 3.8), the absorption of light through various human tissues has been found to be quite weak $\left(l_{\mathrm{a}}>10 \mathrm{~mm}\right)$ within a small spectral window spanning from approximately 600 to $1100 \mathrm{~nm}$. Outside this window absorption rises steeply being dominated by Haemoglobin, the red oxygen carrying protein constituent of blood on the short wavelength side and by water on the long wavelength side. ${ }^{34}$ A comparison of scattering and absorption mean-free-path lengths within this spectral 
window shows, that for almost all tissues, scattering is the dominant interaction process and the albedo is, therefore, close to unity $(\gamma \approx 1)$.

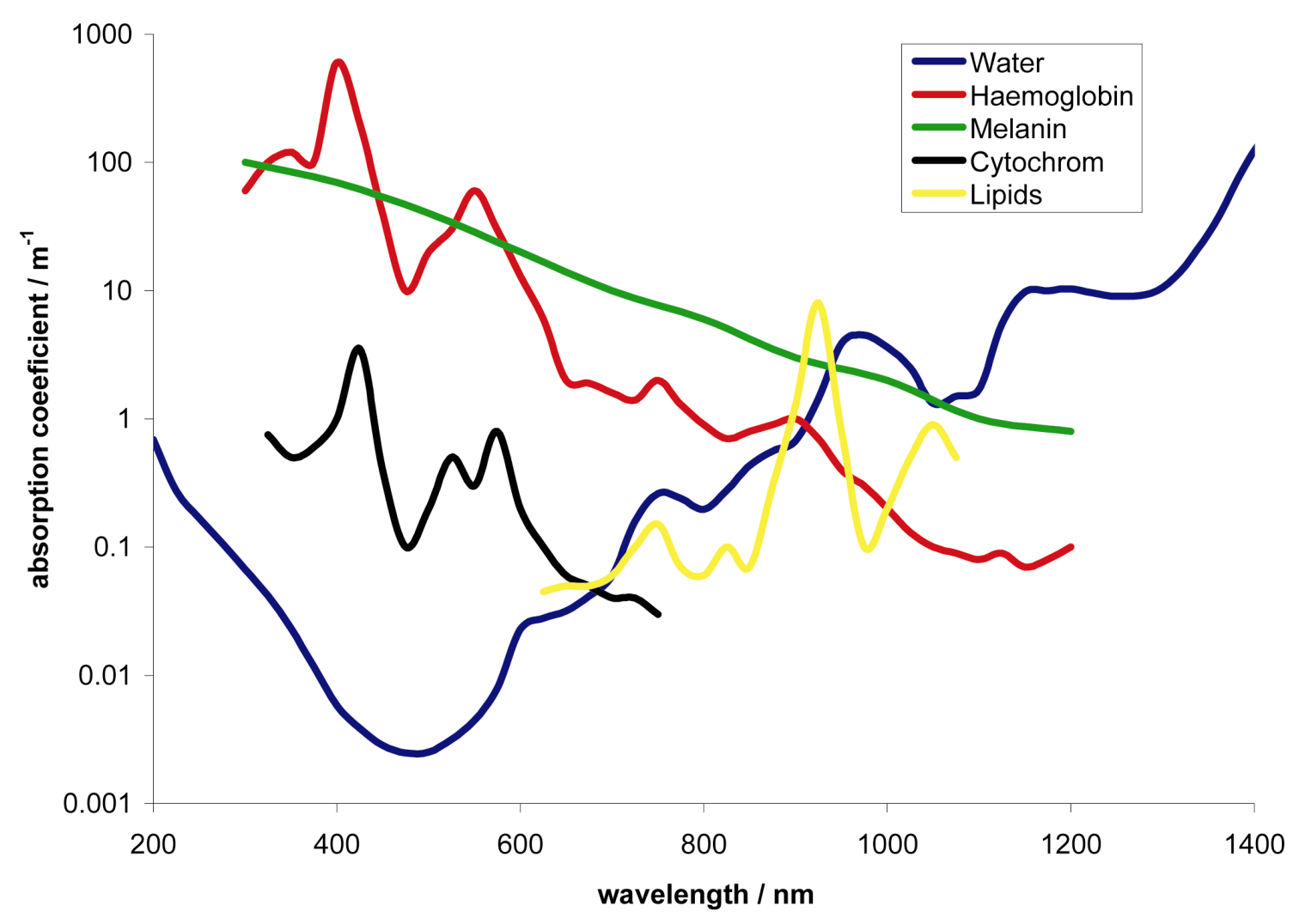

Fig. 3.8 Absorption-spectra for water, and the major tissue chromophores, haemoglobin, melanin, cytochromes, and lipids, in the visible and near infrared. The graph for water applies to a temperature of $298 \mathrm{~K}$ and is taken from Hale \& Querry. ${ }^{35}$ The curves for the tissue chromophores are from Voet \& Voet. ${ }^{36}$

\section{Extinction}

The power of light incident on a thin layer of tissue with thickness $d z$, will be attenuated by a constant fraction $d P / P=-\mu d z$ where $\mu=\mu_{s}+\mu_{a}$ is the total attenuation coefficient. Integration yields the attenuation as a function of layer thickness (Beer-Lambert-Bouguer $\operatorname{law}^{37}$ )

$$
P(z)=P(z=0) e^{-\mu z} .
$$

A warning is in order against incorrect use of the total attenuation coefficient. The formal "attenuation" is often not attenuation in the proper sense but combines absorption and scattering. In contrast to absorbed light, scattered light is, however, not necessarily lost from 
the system but only from a beam propagating in a particular direction and may hence contribute light propagating in other directions. It can even end up back on its initial trajectory by multiple scattering. A contribution of scattered light to the amplitude of unscattered light can thus not be derived from Eqn. 3.12.

\section{Brain tissue (phantom)}

The brain tissue most readily accessible for optical measurements, is the cerebral cortex. In mammals it has six layers arranged in parallel to the surface of the brain and constitutes by far the largest part of the brain. ${ }^{38}$ While the layered structure is essentially defined by the presence or absence of neuronal cell bodies, each layer also contains additional elements such as dendrites and axons. ${ }^{39,40}$ For an extensive reference book on the fine structure of the nervous system see Ramon ${ }^{41}$.

Optically, the cortex appears quite heterogeneous. Apart from the different layer composition, optical differences can also derive from different preferred orientations of neuronal fibres as is found in most layers. ${ }^{41}$ It is hard to provide firm numbers for the optical properties of neocortical tissue. However, the average scattering lengths of human neocortical tissue in the near infrared have been measured in vitro ${ }^{42}$ ranging from $119 \mu \mathrm{m}$ at $700 \mathrm{~nm}$ to $181 \mu \mathrm{m}$ at $1100 \mathrm{~nm}$; the anisotropy factor was measured to be $0.9 \pm 0.025$ and constant to within $2 \%$ over this range ${ }^{\dagger}$. A quantitative evaluation in vivo has been made in cortex of rat where the twophoton fluorescence fall-off of labelled vasculature has been measured. ${ }^{3,43}$ At an excitation wavelength of $830 \mathrm{~nm}$, the decay constant for the two-photon fluorescence with depth was about $100 \mu \mathrm{m}$, suggesting a scattering mean-free-path length of approximately $200 \mu \mathrm{m}$. A recent study ${ }^{5}$ confirmed this and found that the scattering mean-free-path length increases with wavelength (1.5-fold between $760 \mathrm{~nm}$ and $900 \mathrm{~nm})$ in line with the results from in vitro experiments. ${ }^{42}$ In brain slices, Oheim et al. ${ }^{5}$ found that the scattering mean-free-path length is approximately twice as long in juvenile than in adult brain, perhaps because of the incomplete myelinization in juveniles.

Also important in the context of non invasive imaging are the optical properties of tissues overlying the cortex i.e. skin, skull and meninges (the lining membranes of the cortex). Skin is a strong absorber and scatterer and usually needs to be removed from the imaging field. Skull, although exhibiting optical properties similar to that of cortex, ${ }^{44}$ degrades the imaging depth

\footnotetext{
${ }^{\dagger}$ Data taken from plots.
} 
significantly because of its relative large thickness and is thus usually thinned if not completely removed. Of the three meninges, it is only the dura mater, a dense fibrous membrane closely adherent to the scull, which in some mammals is particularly hostile to the propagation of light and in such cases is removed from the imaging field. ${ }^{45,46}$

In order to replicate the optical properties of neocortical tissue, we designed a phantom consisting of low melting-point agarose (0.5\%, A-2576, Sigma Inc.) containing $0.992 \mu \mathrm{m}$ non-fluorescent and $1.011 \mu \mathrm{m}$ yellow-green (490/515) fluorescent polystyrene scattering beads (Polyscience Inc.) at a total concentration of $5.3 \cdot 10^{9}$ beads $/ \mathrm{ml}$. The use of agarose as base material (99.5\% of the total volume) with a refraction index only slightly above 1.33 ensures that the volume weighted average of the refraction index for agarose and scattering beads (the refraction index of polystyrene is 1.56) will not much deviate from 1.33 and thus from that found for brain tissue. The use of fluorescent and non-fluorescent beads of equal size allows to simulate different tissue staining fractions by changing their relative portions, leaving the total bead concentration - which sets the scattering free-mean-path length unchanged. The scattering length and anisotropy at an excitation wavelength of $909 \mathrm{~nm}$ were calculated using Mie theory to be 0.88 and $200 \mu \mathrm{m}$, respectively $(0.94$ and $77 \mu \mathrm{m}$ at $515 \mathrm{~nm}$, the fluorescence wavelength).

The scattering mean-free-path length was verified by measuring the spectral transmission through a $160 \mu \mathrm{m}$ thick slab (see Fig. 3.9) using a spectrophotometer (1 mm beam size, Cary 500, Varian, Inc.). The transmission at $909 \mathrm{~nm}$ was 0.455 corresponding to a scattering freemean-path length of $203 \mu \mathrm{m}$, close to the expected value.

As mentioned above, two-photon fluorescence microscopy has been used to infer the size of the scattering mean-free-path length from in vivo experiments. ${ }^{3,43}$ To demonstrate the feasibility of this method, we have imaged fluorescent beads from the phantom (the ratio of fluorescent to non-fluorescent beads was 1/10) using a two-photon microscope. With depth, the two-photon fluorescence $(F)$ generated in the beads should fall with the square of the excitation power, i.e. $F \propto\left[\bar{P}_{0} \mathrm{e}^{-z / l_{s}}\right]^{2} \propto \mathrm{e}^{-2 z / l_{s}}$. We found that the fluorescence signal measured from the phantom decayed exponentially with depth (see Fig. 3.10); the attenuation length constant of $94.6 \pm 4.6 \mu \mathrm{m}$ being very close to the expected value $\left(l_{\mathrm{s}} / 2=100 \mu \mathrm{m}\right)$. 


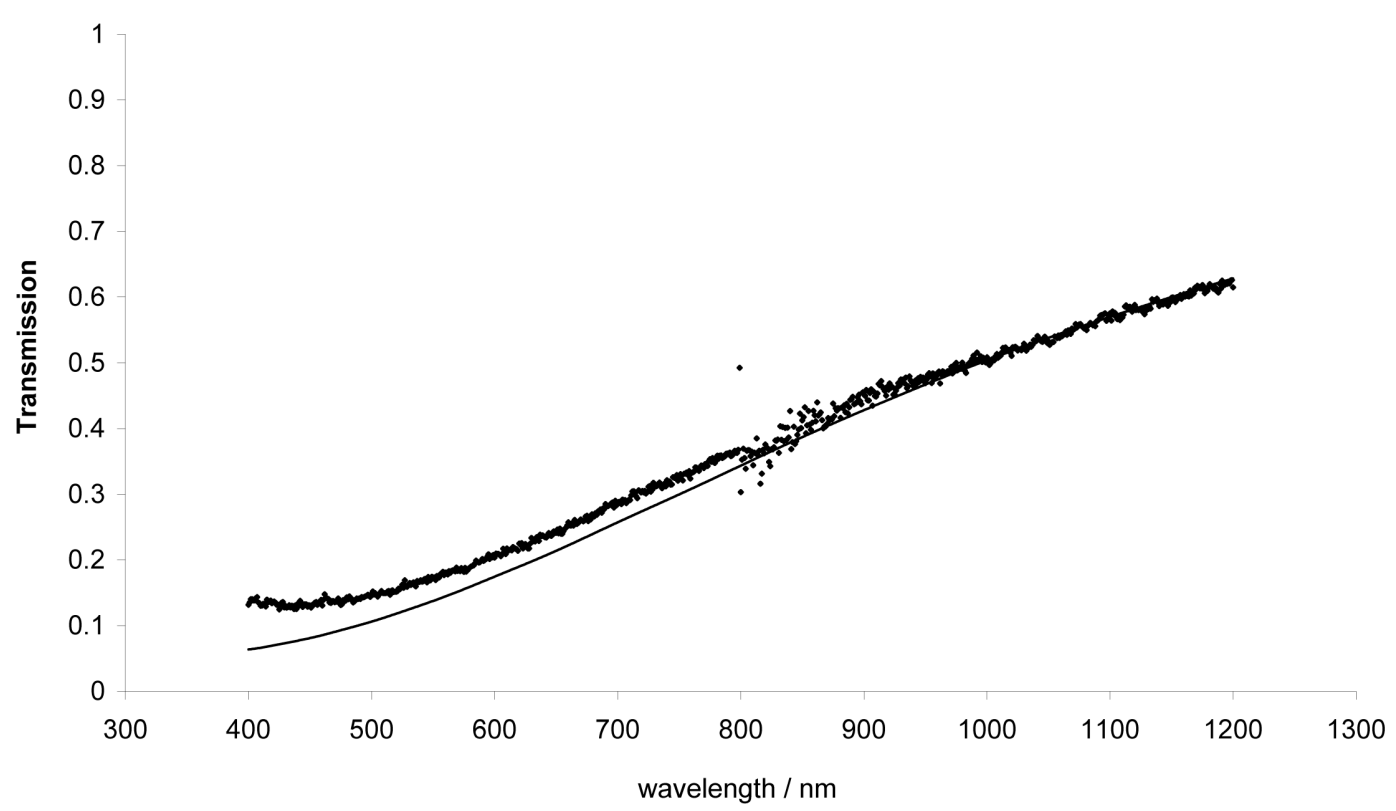

Fig. 3.9 Spectral transmission through a $160 \mu \mathrm{m}$ thick agarose slab containing $0.992 \mu \mathrm{m}$ non-fluorescent polystyrene beads at a concentration of $5.3 \cdot 10^{9}$ beads $/ \mathrm{ml}$. Solid line shows what is expected from Mie theory. The resolution and integration time of the spectrophotometer used (Cary 500, Varian, Inc.) were $1 \mathrm{~nm}$ and $1 \mathrm{~s}$, respectively. The beam diameter was reduced to $1 \mathrm{~mm}$ using two pin holes placed before and after the slab. The different noise levels apparent on the spectrometer curve are due to different detectors used for the visible and near infrared range (detector change over point was at $800 \mathrm{~nm}$ ).

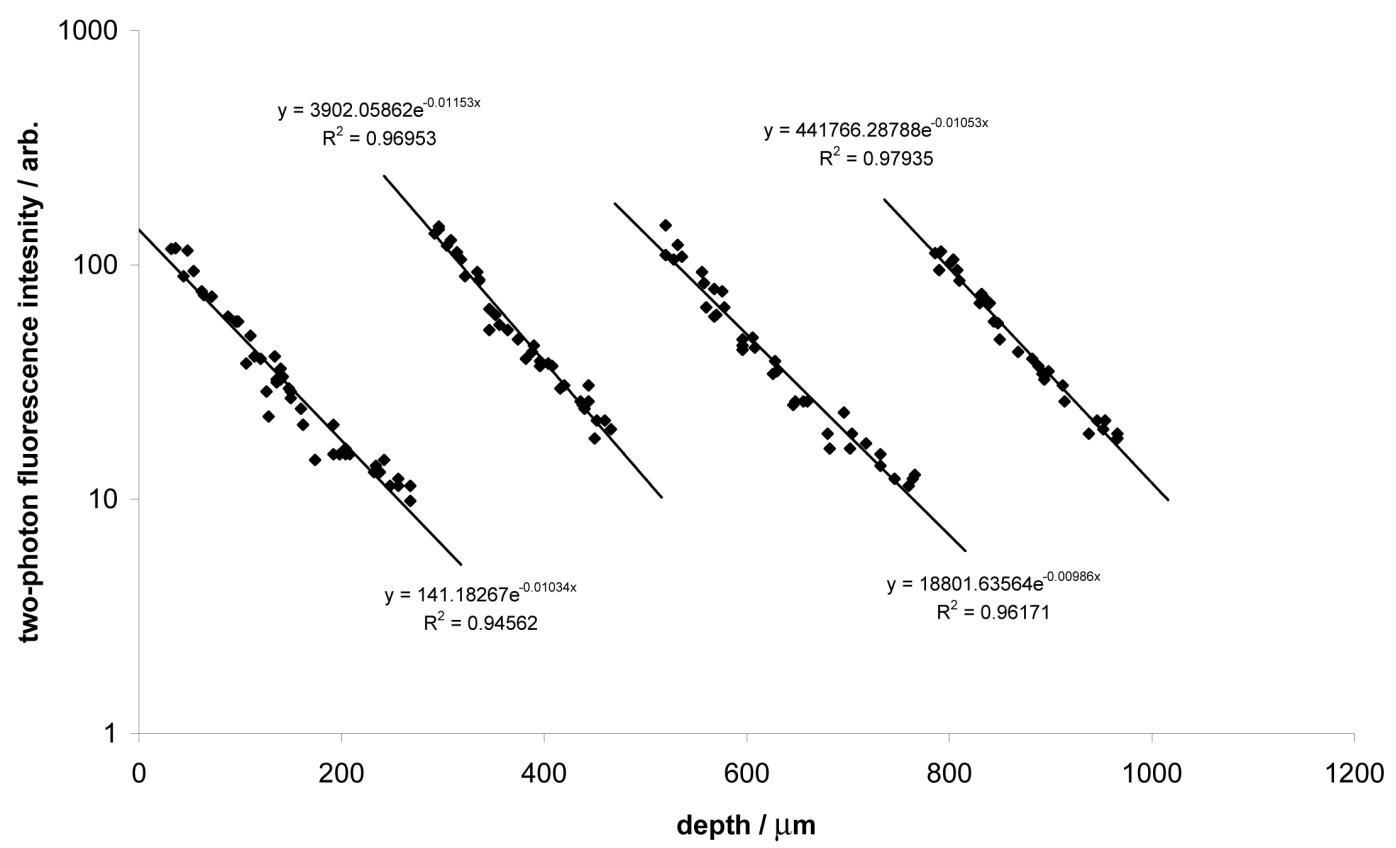

Fig. 3.10 Background corrected two-photon fluorescence intensity versus imaging depth for constant incident power. The fluorescence intensity from fluorescent beads in a tissue phantom (see text) was measured by acquiring planar scans at successive depths from 0 to $1000 \mu \mathrm{m}$. Fluorescence intensity steps in the data correspond to manual resets of the incident power performed when the bead fluorescence intensity fell close to the dark current level of the image. Solid lines are best fits of $F=F(0) e^{-x / l}$ to the data yielding an average decay constant of $l=94.6 \pm 4.6 \mu \mathrm{m}$. 
Next, we acquired an image stack consisting of 415 z-sections (x-y scans) of 256x256 pixels of a phantom with a large staining ratio as is often encountered in vivo. Images were averaged over 3 scans at a frame rate of 1.95/s and taken at depth increments of $3 \mu \mathrm{m}$ down to a depth of $1150 \mu \mathrm{m}$. In order to maintain a roughly depth-independent signal strength, the incident power was continuously adjusted according to $P=P(0) e^{z / l_{s}}$ with $l_{\mathrm{s}}$ pre-set to $200 \mu \mathrm{m}$.

A maximum projected view $(x-z)$ of a small sub-volume of the tissue phantom and single $x-y$ scans at different depths $z$ are shown in Fig. 3.11.

As can be seen, the bead fluorescence signal could be maintained over the entire volume, using only about $30 \%$ of the available laser power at the deepest focus setting. At depths below $1000 \mu \mathrm{m}$, however, the image quality deteriorate rapidly due to a steep rise in background fluorescence. The maximum imaging depth was, therefore, not limited by the available excitation power but by an increase in out-of-focus fluorescence, which is presumably mainly generated near the surface. ${ }^{12}$ The reason for this increase is, that in order to compensate for the scattering loss of excitation the incident power needs to be increased exponentially with depth. This exponential increase eventually prevails over the power-law fall-off of the excitation efficiency due to the increased beam cross-sectional area $\left(A \approx \pi z^{2}(N A)^{2} / n^{2}\right)$. This then leads to a steep increase in excitation efficiency at and near the surface $F_{\text {surface }} \propto P^{2} / A \propto e^{2 z / l_{s}} /\left(\pi z^{2}(N A)^{2} / n^{2}\right)$. At some point, out-of-focus fluorescence will therefore inevitably dominate the total fluorescence generated resulting in a loss of image contrast.

One possible definition of the maximum imaging depth is the point at which the signal-tobackground ratio becomes unity (see section 4.4). Using this criterion, the maximum imaging depth in the tissue phantom was about $860 \mu \mathrm{m}$. 


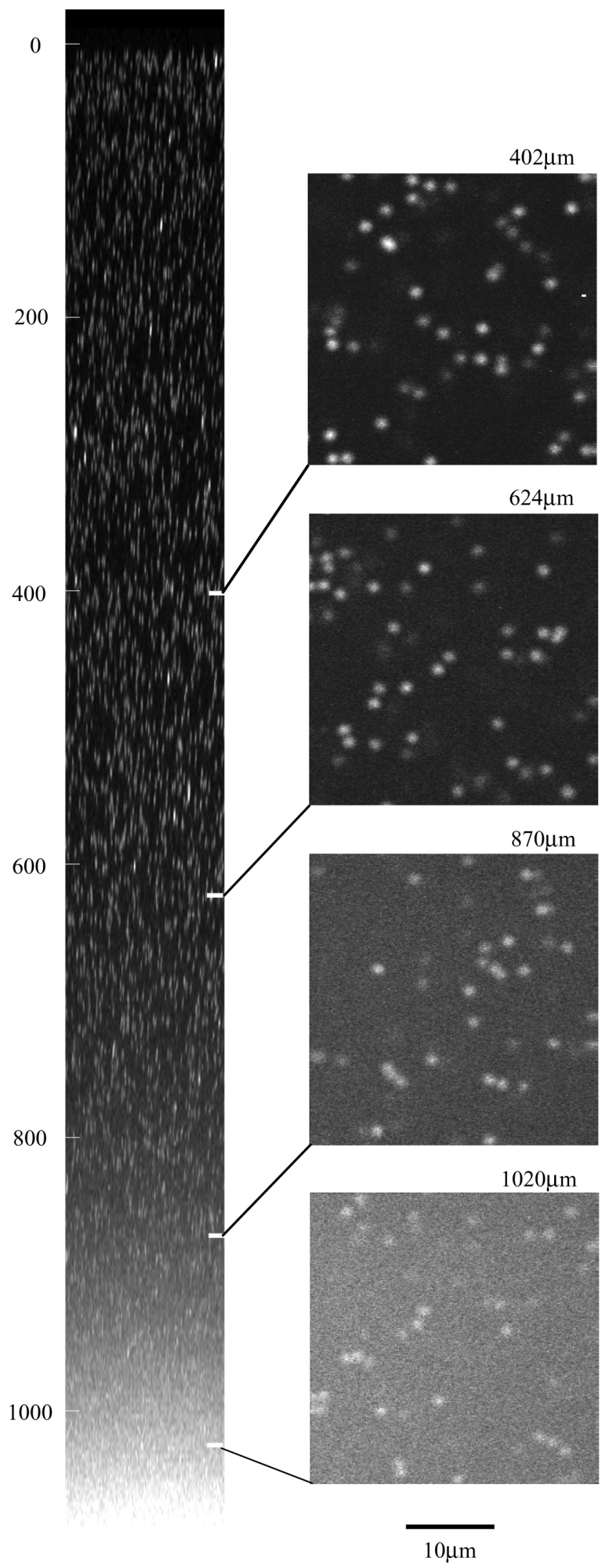

Fig. 3.11 $x$-z projection of the fluorescent bead distribution in a sub-volume of the described brain tissue phantom and single planar $x-y$ scans at four different depths $(z)$. 


\subsubsection{In vivo measurements}

\section{Imaging morphology}

Two staining modalities were used for in vivo measurements. In the first, we injected a fluorescence tracer (Fluorescein Isothio-Cyanate-Dextran FD-10S, Sigma) into the circulatory system of six-week old mice (C57/BL6, Charles River Laboratories Germany) and thus imaged vasculature and in the second, we measured the distribution of GFP labeled neurons in the neocortex of three-week old transgenic mice (G3-line $\left.{ }^{47}\right)$.

The mice were anaesthetized with $0.14 \%$ (wt/wt) urethane in physiological saline injected intraperitoneally. Their body temperature was monitored by a rectal probe and maintained at $37{ }^{\circ} \mathrm{C}$ throughout the experiment. A metal plate with a quadratic opening of $2 \times 2 \mathrm{~mm}$ above the somatosensory cortex, was attached to the skull to keep the head fixed. A craniotomy was prepared leaving the dura mater intact. To prevent the tissue from drying out the cortical surface was covered with Hepes-buffered Ringer solution ( $\mathrm{pH}$ : 7.2, containing (in $\mathrm{mM}$ ): Hepes: 5.0, $\mathrm{NaCl}: 135, \mathrm{KCl}: 5.4, \mathrm{MgCl} 2$ : 1.0 and $\mathrm{CaCl} 2$ : 1.8 ), which also served as the immersion medium. Blood serum was labeled by tail-vein injection of $100 \mu \mathrm{lof} 5 \%$ (wt/vol.) Fluorescein Isothio-Cyanate-Dextran FD-10S (Sigma Inc.) in physiological saline. In order to minimize wavefront distortion and the generation of out-of-focus fluorescence, special care was taken to avoid imaging through big blood vessels, which are frequently found in the superficial layers above the pia-mater.

As in the phantom, the fluorescence signal strength could be maintained throughout the entire volume by increasing the incident power exponentially with depth (see above), using a length constant in the range from 200 to $250 \mu \mathrm{m}$. Maximum-value projections of the scanned volumes along the $x$ axes and $x-y$ scans at 3 different depths (z-positions) are shown in Fig. 3.12 (stained vasculature) and Fig. 3.13 (GFP-labeled neurons). For both staining modalities, the imaging depth was not limited by the available excitation power but, as in the phantom, by out-of-focus fluorescence. The maximum imaging depth, using the definition given above, was about $850 \mu \mathrm{m}$. Down to this depth, individual capillaries and neurons with diameters of 5 $\mu \mathrm{m}$ and $10 \mu \mathrm{m}$, respectively, could be resolved. While at depths $>850 \mu \mathrm{m}$, image quality rapidly deteriorates, neurons and blood vessels could be detected even at depths beyond 1000 $\mu \mathrm{m}$, albeit with poor contrast. 


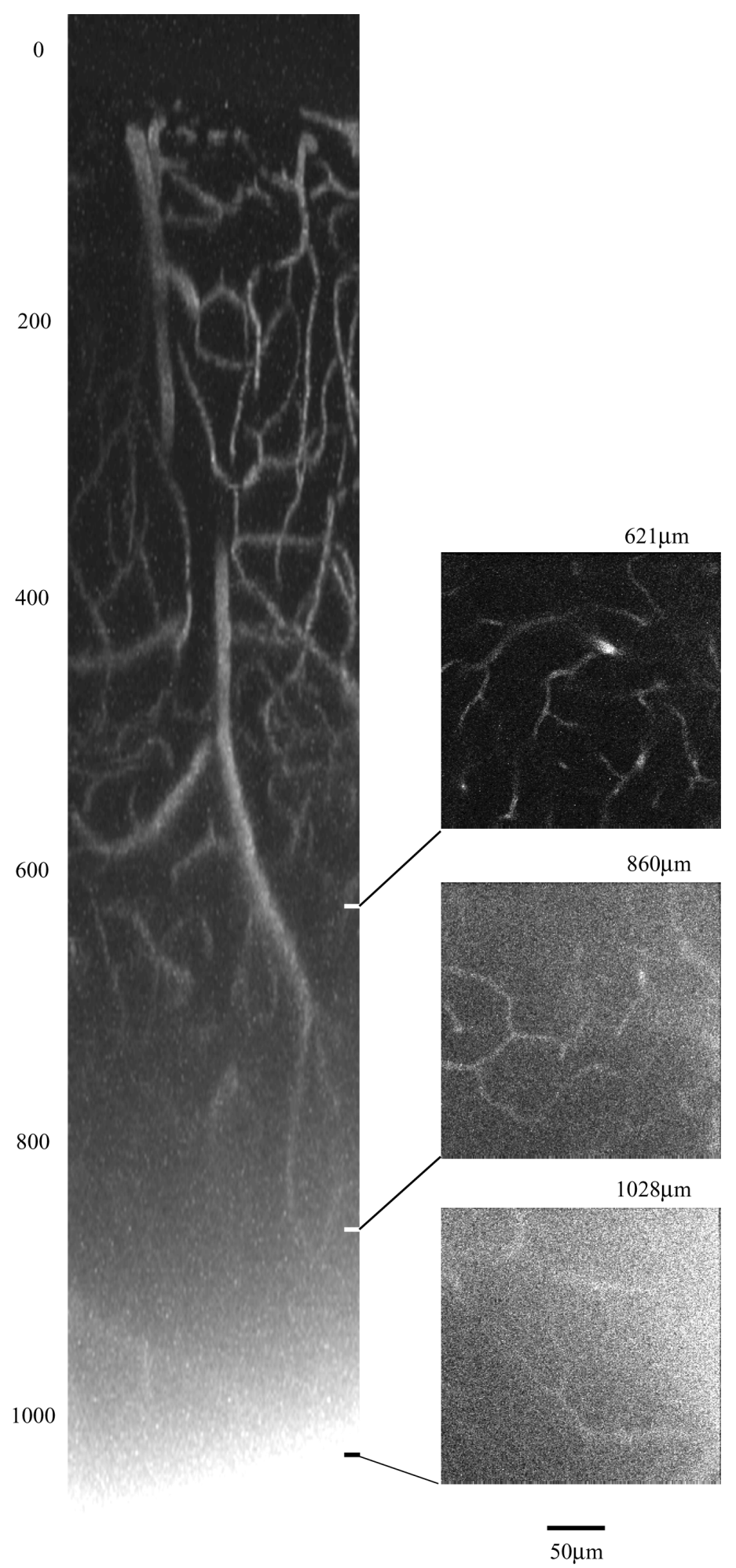

Fig. $3.12 x-z$ projection of stained vasculature in mouse neocortex and single planar $x-y$ scans at three different depths $(z)$. 


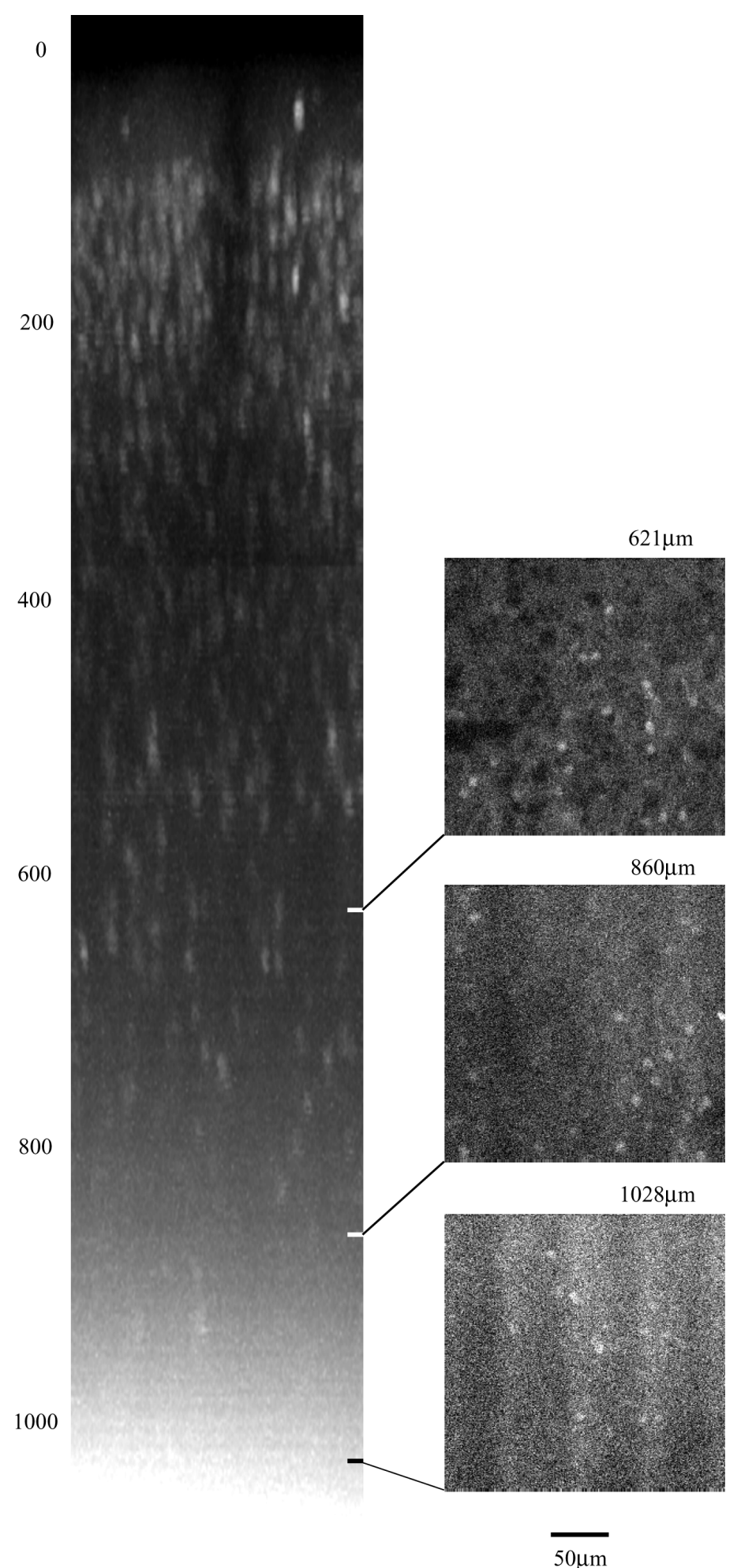

Fig. $3.13 x-z$ projection of GFP labeled neurons in mouse neocortex and single planar $x-y$ scans at three different depths $(z)$; vertical striping in the deepest $x-y$ image is due to heartbeat induced brain motion. 


\section{Imaging physiology}

As an example for obtaining functional signals, we measured the flow of red blood cells in individual capillaries. This can be achieved by fluorescent labeling of blood plasma in the same manner as for the structural imaging of vasculature (see above). Since red blood cells remain unstained, they appear as dark objects on an otherwise bright background.

The first measurements of the micro-circulation were performed at the surface and superficial layers of the rat brain using confocal microscopy. ${ }^{48,} 49$ However, using confocal microscopy, the maximum imaging depth is limited to about $250 \mu \mathrm{m}$. Depths of more than $600 \mu \mathrm{m}$ below the pia mater, down to the fourth cortical layer, were achieved using two-photon microscopy. ${ }^{3}$ In order to demonstrate that functional imaging at even larger depths can be achieved using optically amplified pulses, we measured the motion of red blood cells through individual capillaries at depths of 750 and $900 \mu \mathrm{m}$. Line scans along the central axis of the capillaries (for both capillaries the diameter was about $5 \mu \mathrm{m}$ ) have been repeatedly taken to produce a continuous measure of red blood cell velocity (see Fig. 3.14). While the spatial coordinate was taken to be along the direction of the scan i.e. along the selected capillary section, the perpendicular direction represents the temporal coordinate. The motion of a red blood cell through the capillary appears as a shadow changing its position between subsequent scans (proportional to its instantaneous speed) tracing out a shaded band (see Fig. 3.14) in the continuous flow image. The slope of the band $(\Delta t / \Delta s)$ is inversely proportional to the velocity, the distance between bands at a fixed time $(d x)$ is proportional to the linear density and the time between bands at a fixed position $(d t)$ is inversely proportional to the flux of red blood cells.

Before discussing the results, I would like to emphasize that the main goal of these measurements was to show the feasibility of using optically amplified laser pulses for imaging physiology in vivo at depths that were hitherto not accessible. Since data were obtained from only two capillaries, conclusions pertaining to general blood flow characteristics should be drawn with caution. For the measurement on the capillary at a depth of $750 \mu \mathrm{m}$, we observed a large variability in the flow parameters. Such a behavior is not unusual and has been associated with vessels in T-junction configurations (for more examples and a discussion see Kleinfeld et al. ${ }^{3}$ ). In the following we will focus on the analysis of the data obtained from the capillary at $900 \mu \mathrm{m}$ in which, in contrast to the capillary at $750 \mu \mathrm{m}$, there were only very small variations in the flow parameters. From the continuous flow image we determined the 
velocity, linear density and flux to be $0.71 \mathrm{~mm} \mathrm{~s}^{-1}, 66.7 \mathrm{~mm}^{-1}$ and $46.5 \mathrm{~s}^{-1}$, respectively. The average spacing between red blood cells given by the ratio of velocity to flux was $15.3 \mu \mathrm{m}$. These values are in line with previously reported data. ${ }^{3}$
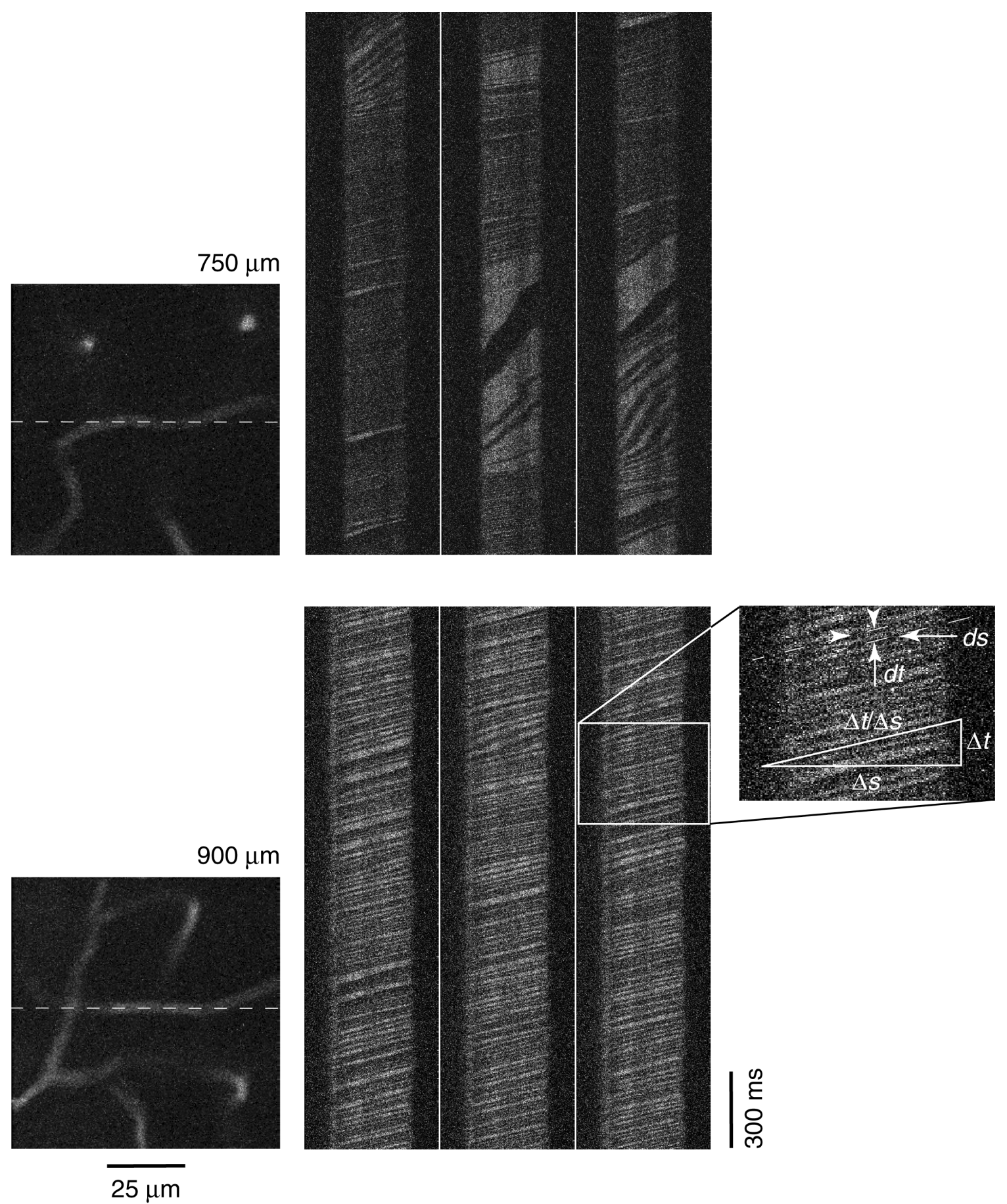

Fig. 3.14 Blood flow measurements: single planar scans $(x-y)$ at depths of $750 \mu \mathrm{m}$ (top) and $900 \mu \mathrm{m}$ (bottom) (line scan positions indicated by dashed lines) and respective continuous flow images (spatial coordinate scaled to $50 \%$ of the single planar scans). Inset (magnification $200 \%$ ) used for the calculation of red blood cell velocity, linear density and flux $(d s \approx 15 \mu \mathrm{m}, d t \approx 21 \mathrm{~ms}, \Delta t / \Delta s \approx 1.41 \mathrm{~s} / \mathrm{mm})$. 


\subsection{Conclusions}

Using a regenerative amplifier as the excitation source, the maximum imaging depth in twophoton microscopy can be substantially increased. Stained vasculature and GFP-labeled neurons can be resolved down to a depth of about $1000 \mu \mathrm{m}$ below the brain surface in the cortex of mice in vivo. Functional signals have been obtained down to a depth of $900 \mu \mathrm{m}$ : the motion of red blood cells in individual capillaries with a diameter of about $5 \mu \mathrm{m}$ has been measured. In all cases, the maximum imaging depth was not limited by the available excitation power but by out-of-focus fluorescence, generated mainly near to the surface.

In the following chapter, I will aim to quantify this fundamental limitation in imaging depth in two-photon microscopy by investigating the generation and detection of focus and out-offocus fluorescence separately. 


\section{References}

1. P. Theer, M. T. Hasan, and W. Denk, "Two-photon imaging to a depth of $1000 \mathrm{mu} \mathrm{m}$ in living brains by use of a Ti : Al2O3 regenerative amplifier," Optics Letters 28(12), 1022-1024 (2003).

2. P. Theer, M. T. Hasan, and W. Denk, "Multi-photon imaging using a Ti : sapphire regenerative amplifier," in Confocal, Multiphoton, and Nonlinear Microscopic Imaging, Volume 5139 (SPIE, 2003), pp. 1-6.

3. D. Kleinfeld, P. P. Mitra, F. Helmchen, and W. Denk, "Fluctuations and stimulusinduced changes in blood flow observed in individual capillaries in layers 2 through 4 of rat neocortex," Proceedings of the National Academy of Sciences of the United States of America 95(26), 15741-15746 (1998).

4. K. Svoboda, F. Helmchen, W. Denk, and D. W. Tank, "Spread of dendritic excitation in layer 2/3 pyramidal neurons in rat barrel cortex in vivo," Nature Neuroscience 2(1), 65-73 (1999).

5. M. Oheim, E. Beaurepaire, E. Chaigneau, J. Mertz, and S. Charpak, "Two-photon microscopy in brain tissue: parameters influencing the imaging depth," Journal of Neuroscience Methods 111(1), 29-37 (2001).

6. E. Beaurepaire and J. Mertz, "Epifluorescence collection in two-photon microscopy," Applied Optics 41(25), 5376-5382 (2002).

7. M. Muller, J. Squier, R. Wolleschensky, U. Simon, and G. J. Brakenhoff, "Dispersion pre-compensation of 15 femtosecond optical pulses for high-numerical-aperture objectives," Journal of Microscopy-Oxford 191, 141-150 (1998).

8. E. Beaurepaire, M. Oheim, and J. Mertz, "Ultra-deep two-photon fluorescence excitation in turbid media," Optics Communications 188(1-4), 25-29 (2001).

9. S. H. Cho, F. X. Kartner, U. Morgner, E. P. Ippen, J. G. Fujimoto, J. E. Cunningham, and W. H. Knox, "Generation of 90-nJ pulses with a 4-MHz repetition-rate Kerr-lens mode-locked Ti : A12O3 laser operating with net positive and negative intracavity dispersion," Optics Letters 26(8), 560-562 (2001).

10. M. Ramaswamy, M. Ulman, J. Paye, and J. G. Fujimoto, "Cavity-Dumped Femtosecond Kerr-Lens Mode-Locked Ti-A12o3 Laser," Optics Letters 18(21), 18221824 (1993).

11. T. B. Norris, "Femtosecond Pulse Amplification at $250 \mathrm{Khz}$ with a Ti-Sapphire Regenerative Amplifier and Application to Continuum Generation," Optics Letters 17(14), 1009-1011 (1992). 
12. J. P. Ying, F. Liu, and R. R. Alfano, "Spatial distribution of two-photon-excited fluorescence in scattering media (vol 38, pg 224, 1999)," Applied Optics 38(10), 21512151 (1999).

13. W. H. Knox, "Femtosecond Optical Pulse Amplification," Ieee Journal of Quantum Electronics 24(2), 388-397 (1988).

14. P. Maine, D. Strickland, P. Bado, M. Pessot, and G. Mourou, "Generation of Ultrahigh Peak Power Pulses by Chirped Pulse Amplification," Ieee Journal of Quantum Electronics 24(2), 398-403 (1988).

15. J.-C. Diels and W. Rudolph, Ultrashort Laser Pulse Phenomena (Academic Press, San Diego, 1996).

16. W. Denk, "Optical beam power controller using a tiltable birefringent plate," U.S. Patent No.: US 6,249,379 B1 (2001).

17. V. P. Kalosha, M. Muller, J. Herrmann, and S. Gatz, "Spatiotemporal model of femtosecond pulse generation in Kerr-lens mode-locked solid-state lasers," Journal of the Optical Society of America B-Optical Physics 15(2), 535-550 (1998).

18. P. Horowitz and W. Hill, The Art of Electronics, 2 ed. (Cambridge University Press, New York, 1989), pp. 508-509.

19. F. Liu, K. M. Yoo, and R. R. Alfano, "Speed of the coherent component of femtosecond laser pulses propagating through random scattering media," Optics Letters 16(6), 351-353 (1991).

20. J. Mobley and T. Vo-Dinh, "Optical Properties of Tissue," in Biomedical Photonics Handbook, V.-D. T., ed. (CRC Press, Boca Raton, 2003).

21. B. Alberts, D. Bray, J. Lewis, M. Raff, K. Roberts, and J. D. Watson, Molecuar Biology of the Cell (Garland Publishing, Inc., New York, 1994).

22. J. W. L. R. Strutt, "On the scattering of light by small particles.," Philosophical Magazine 41, 447-451 (1871).

23. G. Mie, "Beiträge zur Optik trüber Medien, speziell kolloidaler Metallösungen," Annalen der Physik 4(25), 377-445 (1908).

24. M. Born and E. Wolf, Principles of optics (University Press, Cambridge, 1999).

25. H. C. van de Hulst, Light Scattering by small Particles (Dover Publications, Inc., New York, 1981).

26. J. M. Schmitt and G. Kumar, "Optical scattering properties of soft tissue: a discrete particle model," Applied Optics 37(13), 2788-2797 (1998). 
27. V. I. Haltrin, "One-parameter two-term Henyey-Greenstein phase function for light scattering in seawater," Applied Optics 41(6), 1022-1028 (2002).

28. S. T. Flock, B. C. Wilson, and M. S. Patterson, "Total Attenuation Coefficients and Scattering Phase Functions of Tissues and Phantom Materials at 633-Nm," Medical Physics 14(5), 835-841 (1987).

29. J. R. Mourant, J. P. Freyer, A. H. Hielscher, A. A. Eick, D. Shen, and T. M. Johnson, "Mechanisms of light scattering from biological cells relevant to noninvasive opticaltissue diagnostics," Applied Optics 37(16), 3586-3593 (1998).

30. M. Hammer, A. N. Yaroslavsky, and D. Schweitzer, "A scattering phase function for blood with physiological haematocrit," Physics in Medicine and Biology 46(3), N65N69 (2001).

31. L. C. Henyey and J. L. Greenstein, "Diffuse radiation in the Galaxy," Astrophysics Journal 93, 70-83 (1941).

32. T. L. Troy, D. L. Page, and E. M. Sevick-Muraca, "Optical properties of normal and diseased breast tissues: prognosis for optical mammography," Journal of Biomedical Optics 1(3), 342 (1996).

33. M. J. C. Vangemert, S. L. Jacques, H. Sterenborg, and W. M. Star, "Skin Optics," Ieee Transactions on Biomedical Engineering 36(12), 1146-1154 (1989).

34. S. Wan, J. A. Parrish, R. R. Anderson, and M. Madden, "Transmittance of Nonionizing Radiation in Human-Tissues," Photochemistry and Photobiology 34(6), 679-681 (1981).

35. G. M. Hale and M. R. Querry, "Optical-Constants of Water in 200-Nm to 200-Mum Wavelength Region," Applied Optics 12(3), 555-563 (1973).

36. D. Voet and J. G. Voit, Biochemistry (Jhon Wiley \& Sons, Inc., Toronto, 1990).

37. H. G. Pfeiffer and H. A. Liebhafsky, "The Origins of Beer's Law," Journal of Chemical Education 28, 123-125 (1951).

38. R. H. S. Carpenter, Neurophysiology (Arnold, London, 2003).

39. D. G. Amaral, "The Anatomical Organisation of the Central Nervous System," in Principles of Neural Science, fourth ed., E. R. Kandel, J. H. Schwartz, and T. M. Jessel, eds. (McGraw-Hill, New York, 2000).

40. V. Braitenberg and A. Schuz, Statistics and Geometry of Neuronal Connectivity, 2 ed. (Springer-Verlag, Heidelberg, 1998).

41. S. C. Ramon, Histology of the Nervous System of Man and Vertebrates (Oxford University Press, Inc., New York, 1995). 
42. A. N. Yaroslavsky, P. C. Schulze, I. V. Yaroslavsky, R. Schober, F. Ulrich, and H. J. Schwarzmaier, "Optical properties of selected native and coagulated human brain tissues in vitro in the visible and near infrared spectral range," Physics in Medicine and Biology 47(12), 2059-2073 (2002).

43. D. Kleinfeld, P. P. Mitra, F. Helmchen, and W. Denk, "Fluctuations and stimulusinduced changes in blood flow observed in individual capillaries in layers 2 through 4 of rat neocortex (vol 95, pg 15741, 1998)," Proceedings of the National Academy of Sciences of the United States of America 96(14), 8307-8307 (1999).

44. F. Bevilacqua, D. Piguet, P. Marquet, J. D. Gross, B. J. Tromberg, and C.

Depeursinge, "In vivo local determination of tissue optical properties: applications to human brain," Applied Optics 38(22), 4939-4950 (1999).

45. K. Svoboda, D. W. Tank, R. A. Stepnoski, and W. Denk, "Two-photon Imaging of Neuronal Function in the Neocortex In Vivo," in Imaging Neurons A Laboratory Manual, R. Yuste, F. Lanni, and A. Konnerth, eds. (Cold Spring Harbor Laboratory Press, Cold Spring Harbor, 2000).

46. D. Kleinfeld and W. Denk, "Two-Photon Imaging of Neocortical Microcirculation," in Imaging Neurons A Laboratory Manual, R. Yuste, F. Lanni, and A. Konnerth, eds. (Cold Spring Harbor Laboratory Press, Cold Spring Harbor, 2000).

47. H. E. Krestel, M. Mayford, P. H. Seeburg, and R. Sprengel, "A GFP-equipped bidirectional expression module well suited for monitoring tetracycline-regulated gene expression in mouse," Nucleic Acids Res 29(7), E39 (2001).

48. A. Villringer, R. L. Haberl, U. Dirnagl, F. Anneser, M. Verst, and K. M. Einhaupl, "Confocal Laser Microscopy to Study Microcirculation on the Rat-Brain Surface Invivo," Brain Research 504(1), 159-160 (1989).

49. U. Dirnagl, A. Villringer, R. Gebhardt, R. L. Haberl, P. Schmiedek, and K. M. Einhaupl, "3-Dimensional Reconstruction of the Rat-Brain Cortical Microcirculation Invivo," Journal of Cerebral Blood Flow and Metabolism 11(3), 353-360 (1991). 


\section{On the fundamental imaging-depth limit in two-photon}

\section{microscopy}

\subsection{Introduction}

When imaging in turbid media at depths much larger than the scattering-mean-free-path length $\left(l_{\mathrm{s}}\right)$, most of the excitation light is scattered before reaching the focus. While the ballistic fraction decays exponentially, the total power of light reaching a certain depth decays only slowly i.e. at most, inversely with depth for depths beyond one transport-mean-free-path length $\left(l_{t}=l_{s} /(1-g)\right)$. This length corresponds to the average distance after which memory of the lights initial direction is completely lost. Hence, even at a depth as small as one scatteringmean-free-path length, is the power of the scattered fraction much larger than that of the ballistic fraction. The peak intensity of the scattered light is, due to its spatial and temporal dilution, much lower than that of the ballistic fraction and thus inefficient in generating higher order non-linear effects such as two-photon fluorescence or second harmonic generation. Higher order non-linear effects occur thus only at the high intensity focus of the ballistic light. However, due to the different decay rates of the ballistic and scattered light, the focal peak intensity of the ballistic light will eventually become smaller than the peak intensity of the scattered light at the focal plane, rendering imaging impossible. This occurs if the focus is at a depth of about one transport-mean-free-path length (about $2000 \mu \mathrm{m}$ for brain tissue), but it is of minor practical concern because surface generated background, caused by the exponential increase in incident power needed so that sufficient ballistic light reaches the focus will lead to the generation of dominant background (out-of-focus) fluorescence at much smaller depths.

While significant generation of out-of-focus fluorescence by the ballistic fraction is limited to a depth of about one half the scattering-mean-free-path length, for the scattered fraction it may extend to a depth of several scattering-mean-free-path lengths as I will show. In samples with strong forward scattering this effect is particularly pronounced and scattered light is, in fact, the main source for the generation of out-of-focus fluorescence (see section 3.2.2). In the following we will investigate the generation and detection of two-photon fluorescence in a 


\subsection{Two-photon fluorescence generation in turbid media}

Since two-photon absorption is a second-order process, its rate - the number of photons absorbed per unit time and per molecule - is proportional to the square of the excitation intensity (see Eqn. 2.1). The number of photons absorbed per unit time $\left(N_{\mathrm{abs}}\right)$ depends further on the two-photon absorption cross-section $(\delta)$, the fluorophore concentration $(C)$ and the illuminated sample volume $(V)$

$$
N_{a b s}(t)=\int \delta C(\mathbf{r}, t) I^{2}(\mathbf{r}, t) \mathrm{d} V
$$

Assuming no stimulated emission or non-radiative decay such as self-quenching (deexcitation of an exited atom or molecular entity by interaction with another atom or molecular entity of the same species in the ground state), the number of fluorescence photons $(F)$ generated per unit time is given by

$$
F(t)=\frac{1}{2} \eta_{2} N_{a b s}(t)=\frac{1}{2} \eta_{2} \delta \int C(\mathbf{r}, t) I^{2}(\mathbf{r}, t) \mathrm{d} V
$$

where $\eta_{2}$ is the fluorescence quantum efficiency of the dye. Determination of $F(t)$ involves the characterisation of the temporal and spatial distributions of the fluorophore and the excitation light intensity. Continuing with the discussion of the roles that ballistic and scattered excitation light plays in the generation of two-photon fluorescence, we will, in the following, investigate them separately.

\subsubsection{Ballistic excitation intensity}

An intensity distribution commonly encountered is the so called Gaussian beam, ${ }^{2}$ which corresponds to the lowest order cavity mode of laser oscillators. As such it satisfies the paraxial Helmholtz equation (wave equation; for a derivation of the Gaussian beam in free space see for example Siegman $^{3}$ ) and its intensity distribution is given by 


$$
I(z, \rho, t)=\frac{2 P(t)}{\pi w^{2}(z)} e^{-\alpha z} e^{-\frac{2 \rho^{2}}{w^{2}(z)}} .
$$

Here $P(t)$ is the optical power, which generally depends on the time $(t), \lambda$ is the wavelength, $z$ and $\rho=\sqrt{x^{2}+y^{2}}$ are the axial and radial distances, respectively, and $w(z)$ is a measure of the beam width defined as the radial distance at which the intensity has dropped to $1 / \mathrm{e}^{2}$ times that on axis and which is given by

$$
w(z)=w_{0} \sqrt{1+\frac{z^{2}}{z_{r}^{2}}},
$$

where $z_{r}$ is the Rayleigh range and $w_{0}=w(z=0)$ is the beam waist given by

$$
w_{0}=\sqrt{\frac{\lambda z_{r}}{\pi}} .
$$

The most prominent feature of this distribution is a circular symmetric Gaussian intensity profile at any transverse plane. This profile has its peak at $\rho=0$ (on axis) and drops monotonically with increasing $\rho$. The beam width contracts to a minimum at the beam waist $\left(w_{0}\right)$ and increases with axial distance $z$ (in the far field $\left(z \gg z_{r}\right)$ linearly with $z$ ) see Fig. 4.1. Equation 4.3 contains only two free parameters $P_{0}$ and $z_{r}$ which are determined by the boundary conditions. All other parameters are related to $z_{r}$ and $\lambda$.

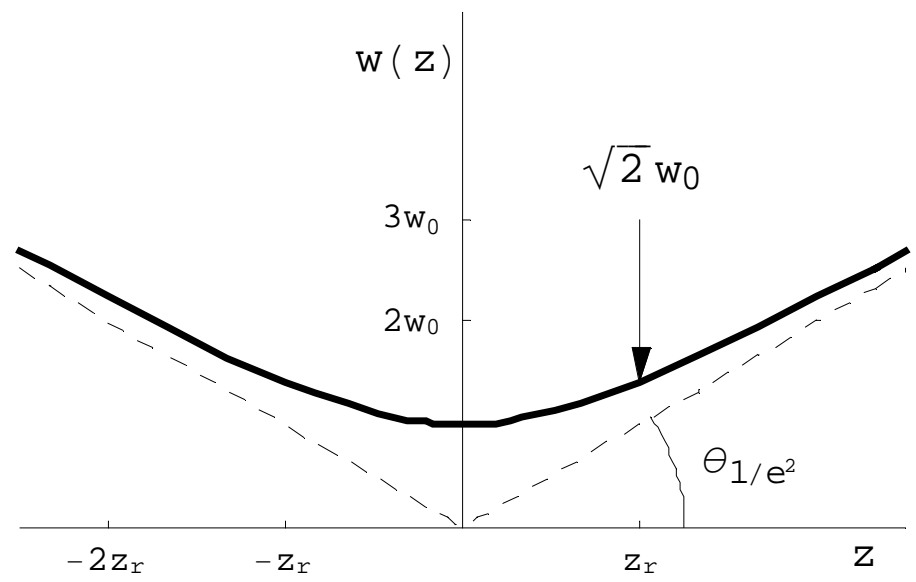

Fig. 4.1 Gaussian beam width $w(z)$ versus axial distance $z$ from the waist $w_{0}$ at $z=0$. The width is $\sqrt{2} w_{0}$ at $z$ $= \pm z_{r}$ (Rayleigh range) and increases linearly with $z$ for large $z$ (far field) defining a cone with half-angle $\theta_{1 / e^{2}}$. 
For a common situation in microscopy where a Gaussian beam is strongly focused by a lens of focal length $f$, the boundary condition for $z_{r}$ can be derived in reverse by viewing the focussing lens as being in the far field of the beams waist placed at the focal plane. As such, the size of the lens' aperture restricts the maximum far-field beam angle that can pass the lens. Defining the far-field beam angle $\theta_{1 / e^{2}}$ to be the angle between the beam contour $w(z)$ and the optical axis as depicted in Fig. 4.1 ( $~ 86 \%$ of the beams power is confined within a cone with this half-angle), the boundary condition takes a very simple form

$$
z_{r}=\frac{w_{0}}{\theta_{1 / e^{2}}}=\frac{\lambda}{n \pi \theta_{1 / e^{2}}^{2}} .
$$

For a Gaussian beam focused into a highly scattering dielectric medium as encountered in most in vivo microscopy applications, the ballistic intensity distribution is somewhat different from that obtained in free space and can be derived from the spherical wave solution to the telegrapher's equation using complex source point co-ordinates and the Fresnel approximation (see appendix). It is given by

$$
I_{\text {ball. }}(z, \rho, t)=\frac{2 n z_{r} P(t)}{\lambda\left(\left(z-z_{0}\right)^{2}+z_{r}^{2}\right)} e^{-\alpha z} e^{-\frac{2 \rho^{2}}{w^{2}(z)}}
$$

with beam parameters

$$
\begin{gathered}
w(z)=2 \sqrt{\frac{\lambda\left(\left(z-z_{0}\right)^{2}+z_{r}^{2}\right)}{4 \pi n z_{r}+\lambda \alpha\left(z-z_{0}\right)}}, \\
w_{0}=\sqrt{\frac{\lambda z_{r}}{n \pi}}
\end{gathered}
$$

where $\alpha=1 / l_{s}$ is the scattering coefficient.

When a Gaussian beam is focussed through a lossless immersion medium $(\alpha=0)$ into the specimen of interest, the beam's Rayleigh range $z_{r}$ (constant throughout the entire thickness of the immersion medium) changes abruptly at the interface of the two media and increases beyond this point monotonically with depth. In contrast, the beam width changes smoothly at the interface and can be calculated at any depth using Eqn. 4.8. The Rayleigh range $z_{r}$, in the 
specimen for a given boundary condition given by the Rayleigh range $z_{r}$ of the beam incident through the immersion medium can be calculated by using Eqn. 4.8 and equating

$$
2 \sqrt{\frac{\lambda\left(z_{0}^{2}+z_{r^{\prime}}^{2}\right)}{4 \pi n z_{r^{\prime}}-\lambda \alpha z_{0}}}=2 \sqrt{\frac{\lambda\left(z_{0}^{2}+z_{r}^{2}\right)}{4 \pi n z_{r}}} .
$$

The first term in Eqn. 4.10 can be viewed as the beam width at the specimen-immersion medium interface $(z=0)$ of a beam travelling from the focus to the surface in an amplifying medium having a different Rayleigh range $z_{r}$, and the second term is the beam width of the unperturbed beam at the interface coming from the same focus. Note, since this follows an inverse approach (going from the focus to the interface) the sign of $\alpha$ has to be changed accordingly (for a lossy medium, $\alpha$ is positive) in Eqn. 4.8. The solution to Eqn. 4.10 is given by

$$
z_{r^{\prime}}=\frac{\sqrt{n \pi}\left(z_{0}^{2}+z_{r}^{2}\right) \pm \sqrt{n \pi\left(z_{0}^{2}-z_{r}^{2}\right)^{2}-\alpha \lambda z_{0} z_{r}\left(z_{0}^{2}+z_{r}^{2}\right)}}{2 \sqrt{n \pi} z_{r}}
$$

Generally, the focus depth is much larger than the Rayleigh range $\left(z_{0} \gg z_{r}\right)$ and Eqn. 4.11 simplifies to

$$
z_{r^{\prime}} \cong\left(1+\left(\frac{\alpha \lambda}{4 n \pi}\right)^{2}\right) z_{r}+\frac{\alpha \lambda}{4 n \pi} z_{0}
$$

Also, $\alpha \lambda /(4 n \pi) \ll 1$ in most practical situations, and Eqn. 4.12 can be further simplified to

$$
z_{r^{\prime}} \cong z_{r}+\frac{\alpha \lambda}{4 n \pi} z_{0}
$$

Hence, the Rayleigh range is no longer a constant but depends on the focal depth $z_{0}$. The same applies to the beam waist which can be seen by substituting $z_{r}$, from Eqn. 4.13 for $z_{r}$ in Eqn. 4.9 giving

$$
w_{0}^{\prime} \cong \sqrt{w_{0}^{2}+\frac{\alpha \lambda^{2} z_{0}}{4 n^{2} \pi^{2}}} .
$$


Note, Eqns. 4.13 and 4.14 describe a reduction of the effective numerical aperture with focal depth $z_{0}$, which, using Eqn. 4.6 and 4.13, and $\theta_{1 / e^{2}}=\beta \theta_{N A}$, is given by

$$
N A \cong N A_{0} / \sqrt{1+\frac{\alpha z_{0}}{4}\left(\beta \frac{N A_{0}}{n}\right)^{2}}
$$

where $\beta$ is the lens fill factor i.e. the ratio of the beam's $1 / \mathrm{e}^{2}$ intensity width at the lens position to the lens aperture radius $\left(\beta=w\left(z=z_{\text {lens }}\right) / R_{\text {lens }}\right)$. This reduction of the effective NA can be explained by the fact, that the peripheral part of the beam experiences a higher attenuation because it has to traverse more material. This effect is naturally more pronounced for larger NAs as can be seen in Figure 4.2.

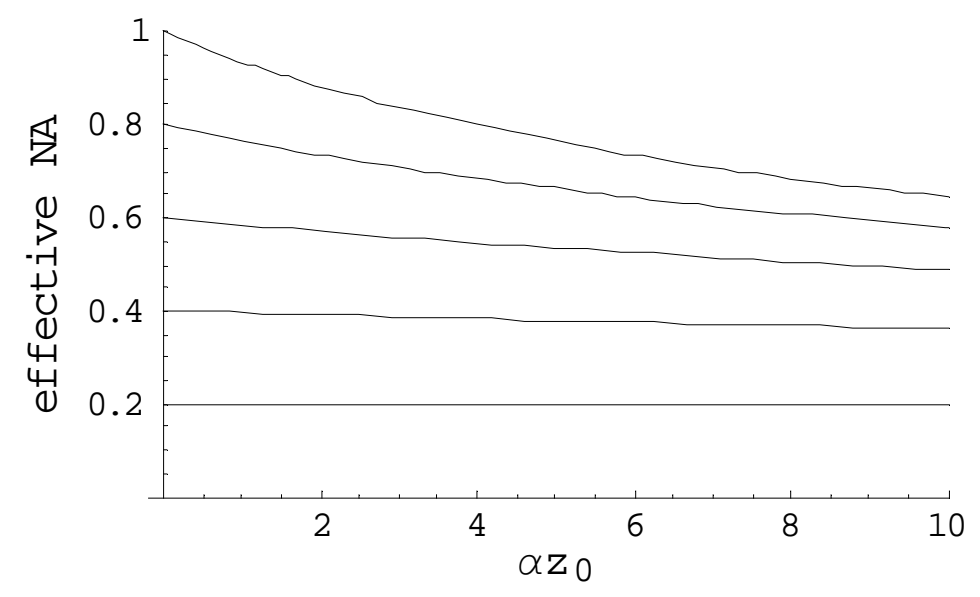

Fig. 4.2 Effective NA versus normalised focus depth $\alpha z_{0}$ for $\beta=1$ and $n=1.33$.

In order to verify Eqn. $4.13-4.15$, one can measure the point-spread function (PSF) comparing predicted and measured $w^{\prime}{ }_{0}$. However, the second term under the root in Eqn. 4.14 is generally small compared to $w_{0}$ (an order of magnitude smaller). Significant changes in $w^{\prime}{ }_{0}$, are only expected for very small $w_{0}$ requiring the use of very high NAs. The simple Gaussian model is, however, only valid if truncation effects are negligible i.e. for a lens fill factor $\beta<$ $0.5{ }^{4}$ Since this reduces the usable NA significantly e.g. for water immersion objectives with the highest suitable NA of 1.0 to $\sim 0.55$, only small increases in $\mathrm{w}^{\prime}{ }_{0}(<10 \%)$ can be expected. In most situations however, such small increases are difficult to measure, in particular when masked by the presence of additional spread factors as wavefront aberrations which lead to similar effects. 
For our point-spread function measurements we have thus used larger lens filling factors taking truncation effects into account. This requires solving the Kirchhoff diffraction integral for a Gaussian pupil function.

\subsubsection{Effective waist and far-field intensity for a truncated Gaussian beam}

Following the approach proposed by Dickson, ${ }^{4}$ the intensity distribution of a Gaussian beam in the focal plane of a lens with focal length $f$ and truncating aperture with radius $R_{\text {lens }}=w\left(z=z_{\text {lens }}\right) / \beta$ is given $\mathrm{by}^{\dagger}$

$$
I(x)=\frac{1}{8 \pi} \beta^{2}\left[e^{-\frac{x^{2}}{4} \beta^{2}}-e^{-\frac{1}{\beta^{2}}} \sum_{m=0}^{\infty}\left\{\frac{\left(-x^{2} / 4\right)^{m}}{m !} \sum_{i=0}^{m} \frac{\left(\beta^{2}\right)^{m-i}}{i !}\right\}\right]^{2}
$$

where $x=2 \pi n \rho /(\lambda f)$ with radial co-ordinate $\rho$.

While the first term in the brackets corresponds to a Gaussian intensity variation, the second term corresponds to the truncation effect. In the case of small truncation $(\beta \rightarrow 0)$, only the first term is significant giving a Gaussian intensity distribution with a width $\left(w_{0}\right)$ according to Eqn. 4.9. For large truncation $(\beta \rightarrow \infty)$, only the second term is significant giving the Fresnel diffraction pattern of a uniformly illuminated circular aperture i.e. an Airy pattern (see Fig. $4.3)$.

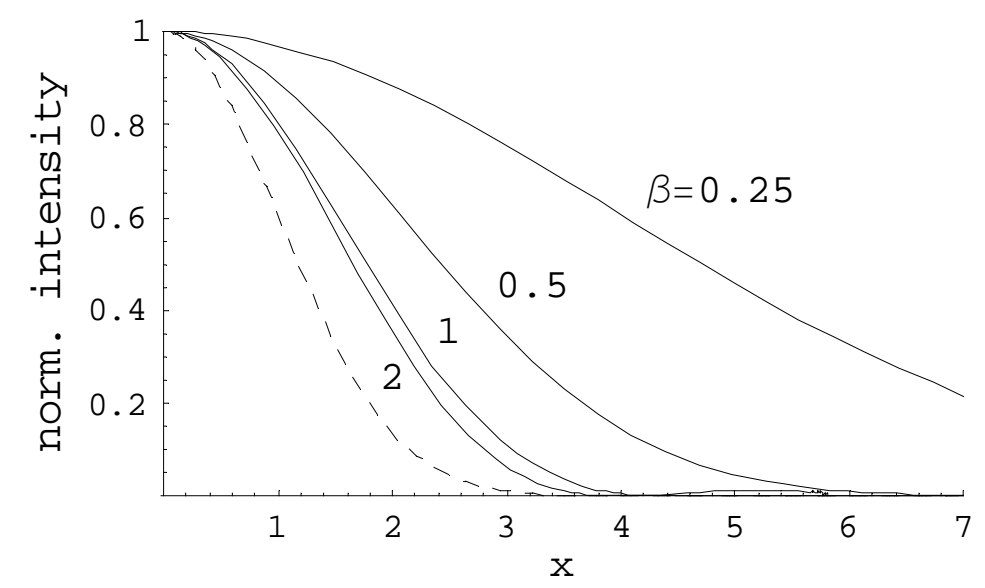

Fig. 4.3 Focal intensity pattern of truncated plane wave Gaussian beams at the lens aperture for various lens fill factors i.e. truncation factors. Note the appearance of side lobes for large $\beta$ marking the transition to an airy pattern. The dashed line is for a non-truncated Gaussian beam with $w\left(z=z_{\text {lens }}\right)=R_{\text {lens }}$.

\footnotetext{
${ }^{\dagger}$ non-absorbing/scattering media are assumed
} 
Using the definition of $1 / \mathrm{e}^{2}$ width for the effective beam waist with truncation $\left(w_{\text {trunc. }}\right)$ over the entire range of $\beta$, we have plotted $w_{\text {trunc. }}$ normalised to the beam waist $w_{0}$ for a non-truncated Gaussian beam with $w\left(z=z_{\text {lens }}\right)=R_{\text {lens }}$ versus $1 / \beta$ (see Fig. 4.4).

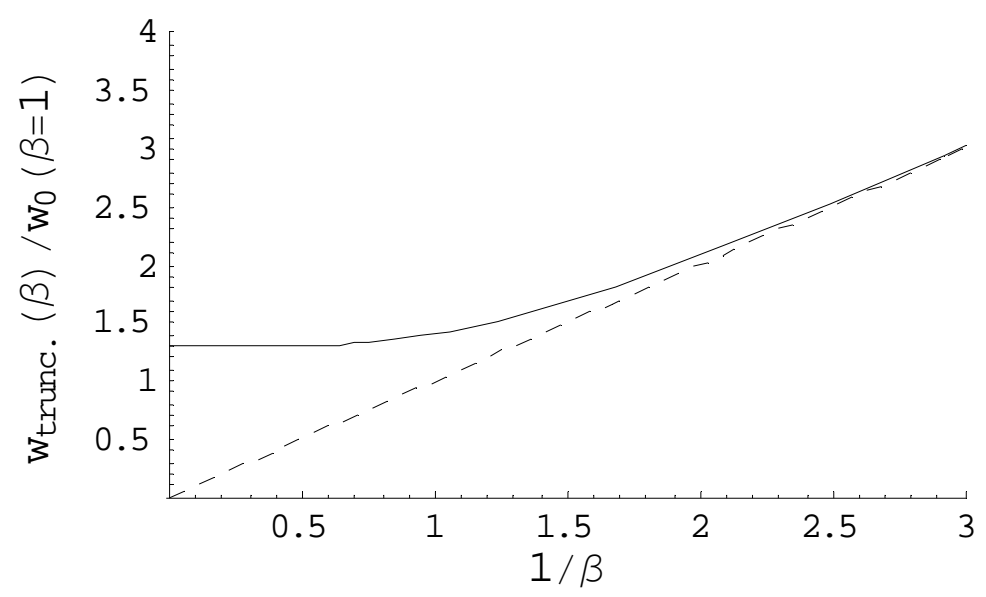

Fig. $4.4 \quad$ Normalised effective beam waist for a truncated Gaussian beam as a function of the inverse lens fill factor (dashed line is for the non-truncated case). The normalisation is to the width of a non-truncated plane wave Gaussian beam with virtual lens fill factor of unity $w_{0}(\beta=1)$ i.e. $w\left(z=z_{\text {lens }}\right)=R_{\text {lens }}$.

While for small lens fill factors the focal beam width is inversely proportional to the lens fill factor, truncation effects become significant for fill factors beyond about 0.5. Characteristic for this transition is the appearance of side-lobes in the intensity pattern and the fact that the beam width asymptotically approaches a constant value corresponding to the $1 / \mathrm{e}^{2}$ width of the Airy intensity pattern. An excellent and analytically simple approximation of this behaviour is given by

$$
\frac{w_{\text {trunc. }}(\beta)}{w_{0}(\beta=1)} \approx a_{0}\left[1+\frac{1}{\left(a_{0} \beta\right)^{4}}\right]^{1 / 4},
$$

where $a_{0}=w_{\text {trunc. }}(\beta \rightarrow \infty) / w_{0}(\beta=1)=1.29$ and $w_{\text {trunc. }}(\beta \rightarrow \infty)$ is the $1 / \mathrm{e}^{2}$ width of the Airy intensity pattern given by

$$
w_{\text {trunc. }}(\beta \rightarrow \infty)=0.67 w_{\text {Airy }}=0.67 \frac{0.61 \lambda}{n \theta_{N A}}=0.41 \frac{\lambda}{n \theta_{N A}}
$$


where $w_{\text {Airy }}$ is the distance to the first null of the Airy intensity pattern. The $1 / \mathrm{e}^{2}$ width of the effective beam waist for a truncated Gaussian beam is thus given by

$$
w_{\text {trunc. }}(\beta) \approx 0.41 \frac{\lambda}{n \theta_{N A}}\left[1+\frac{1}{(1.29 \beta)^{4}}\right]^{1 / 4} \text {. }
$$

Equation 4.19 describes the reduction of the effective NA for a Gaussian beam in the case of truncation (see Fig. 4.5) which, using Eqn. 4.18 and 4.19, is given by

$$
N A_{\text {trunc. }} \approx \frac{0.41 \lambda}{w_{\text {trunc. }}(\beta)} \approx N A_{0} /\left[1+\frac{1}{(1.29 \beta)^{4}}\right]^{1 / 4}
$$

where $N A_{0} \approx 0.41 \lambda / w_{\text {trunc. }}(\beta \rightarrow \infty)$.

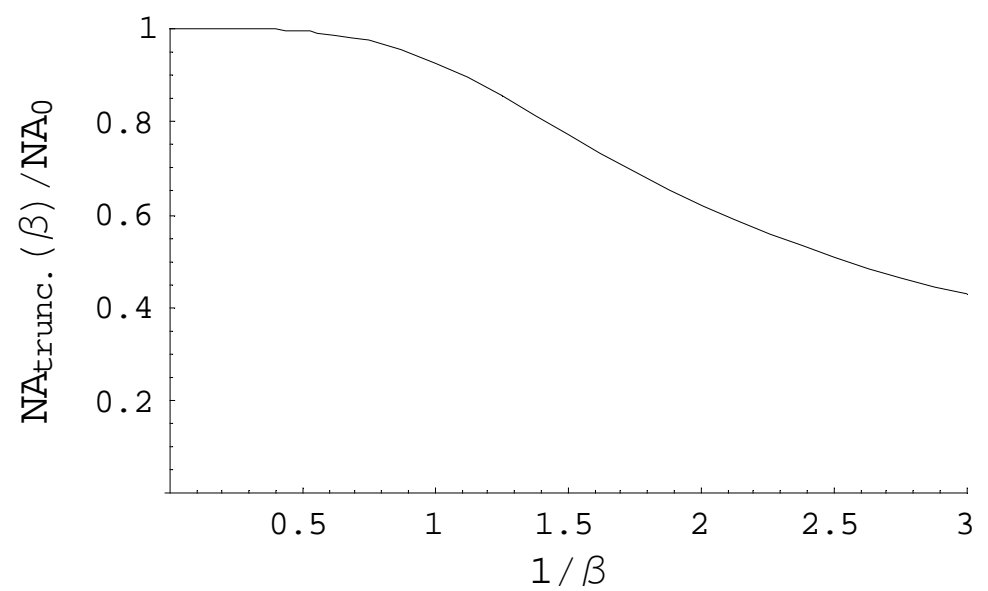

Fig. 4.5 Normalised effective NA for a truncated Gaussian beam as a function of the inverse lens fill factor.

In situations where truncation is significant i.e. $\beta \geq 0.5$ Eqn. 4.14 and 4.15 need to be modified using Eqn. 4.19 and 4.20 and become

$$
\begin{gathered}
w_{0}^{\prime} \cong \sqrt{w_{\text {trunc. }}^{2}+\frac{\alpha \lambda^{2} z_{0}}{4 n^{2} \pi^{2}}}=\frac{\lambda}{n} \sqrt{\frac{0.17}{\theta_{N A}^{2}}\left[1+\frac{1}{(1.29 \beta)^{4}}\right]^{1 / 2}+\frac{\alpha z_{0}}{4 \pi^{2}}} \\
N A \cong N A_{\text {trunc. }} / \sqrt{1+\frac{\alpha z_{0}}{4}\left(\beta \frac{N A_{\text {trunc. }}}{n}\right)^{2}}
\end{gathered}
$$

with $N A_{\text {trunc. }}$ defined by Eqn. 4.20 . 
From Fig. 4.4 it is clear that increasing the lens fill factor decreases the beam width in the focal plane and will thus increase the focal intensity. However, increasing the lens fill factor beyond a certain point $(\sim 0.5)$ will also cause increasing power loss due to truncation at the finite lens aperture and thus a decrease in focal peak intensity. The maximum far field peak intensity that can be achieved is $81.4 \%$ of what could be obtained if the total transmitted power were uniformly distributed over the lens aperture and occurs at $\beta=0.894$ (see Fig. 4.6).

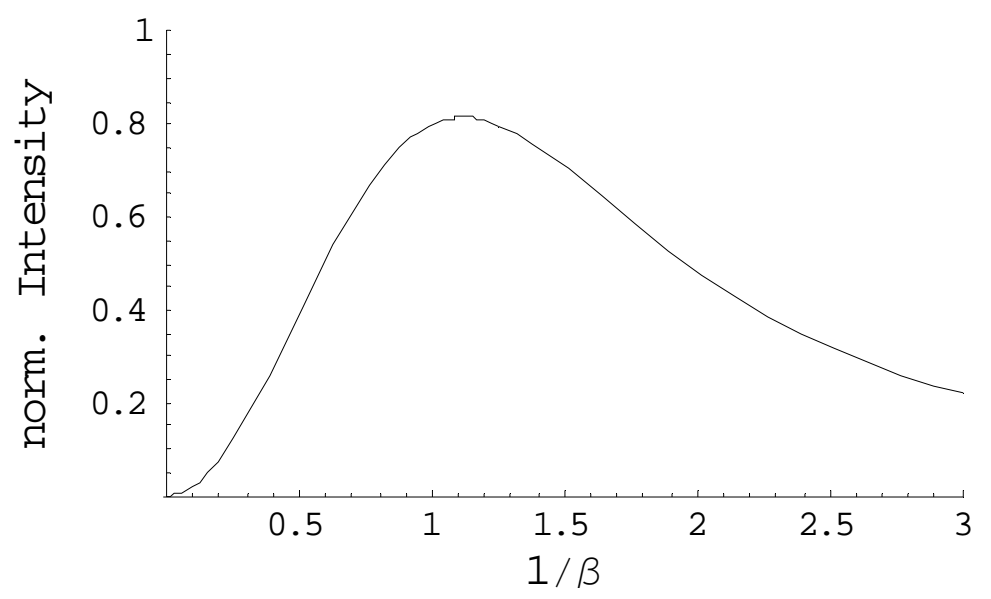

Fig. 4.6 Normalised far-field on axis intensity $I(\beta) e^{-2 / \beta^{\prime 2}} /\left.I\left(\beta^{\prime}\right)\right|_{\beta^{\prime} \rightarrow \infty}$ for a truncated Gaussian beam as a function of inverse lens fill factor.

In order to demonstrate that the expected decrease in the effective NA and, respectively, the increase in focal beam width with depth can indeed be observed, we have measured the pointspread function imaging $93 \mathrm{~nm}$ yellow-green (490/515) fluorescent beads (Molecular Probes, Inc) at a concentration of $2.52 \cdot 10^{11}$ beads $/ \mathrm{ml}$ embedded in a brain tissue phantom containing $0.992 \mu \mathrm{m}$ non-fluorescent polystyrene scattering beads (Polyscience, Inc.) at a concentration of $5.3 \cdot 10^{9}$ beads $/ \mathrm{ml}$ as described in section 3.4.2. At the concentration used, the fluorescent beads did not significantly affect the scattering properties which are dominated by the nonfluorescent beads. Since the fluorescent beads are 9.77 times smaller than the wavelength of the excitation light $(909 \mathrm{~nm})$, they are virtually point sources and the PSF can be obtained directly from recording their three-dimensional fluorescence image.

We measured the PSF at various depths between 0 and $1000 \mu \mathrm{m}$ for two different objectives (a) Olympus XLUMPlanFl 20x/0.95W and (b) Nikon Fluor DIC M 60x/1.0W using the set-up as described in section 3.4.1. The width of the collimated excitation beam at the objectives' back aperture $w\left(z=z_{\text {lens }}\right)$ was determined by measuring the power transmitted through pin- 
holes of different radii $r$. The best fit to the measured data using $1-e^{2 r^{2} / w^{2}\left(z_{\text {lens }}\right)}$ gave a width of $w\left(z_{\text {lens }}\right)=5.42 \mathrm{~mm}$ (see Fig. 4.7).

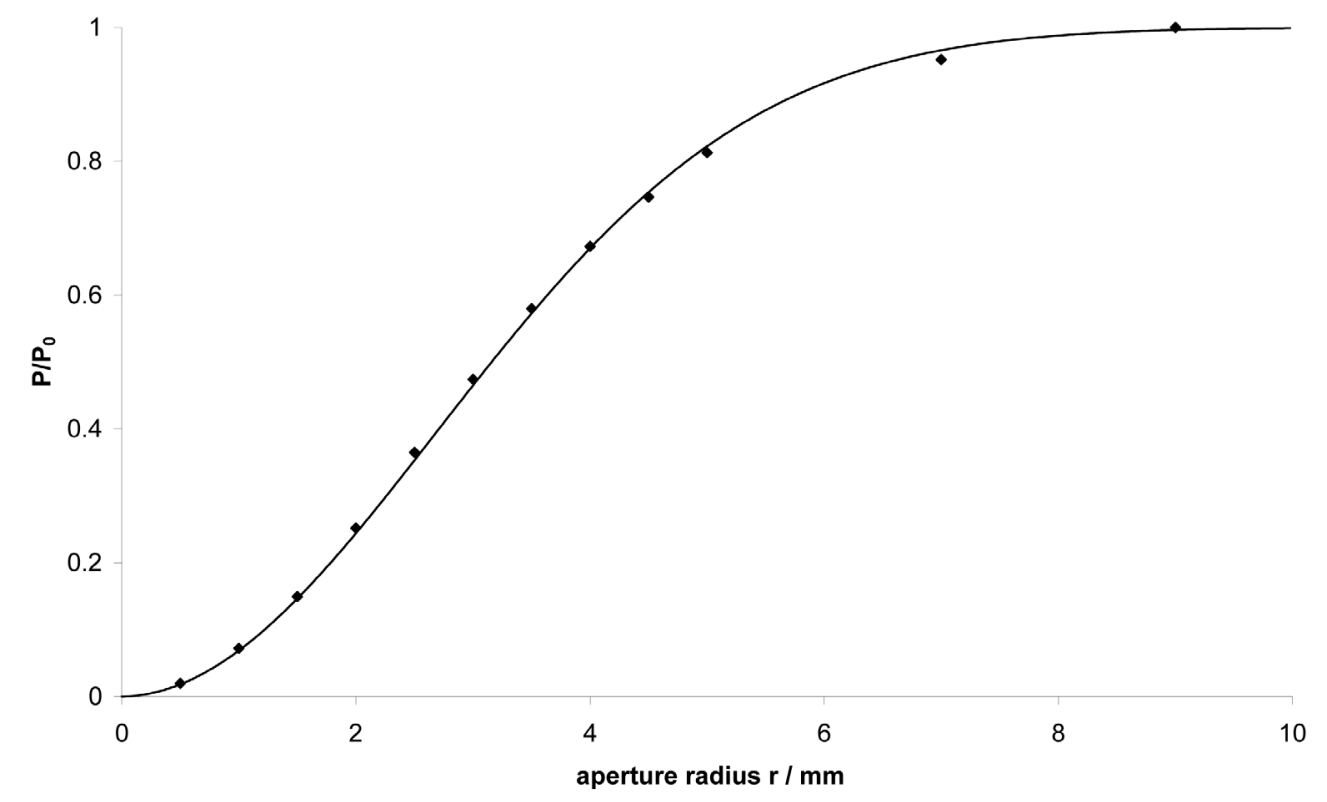

Fig. 4.7 normalised power transmission $\left(P / P_{0}\right)$ as a function of aperture radius $(r)$ used for determining the width of the excitation beam at the objective's back aperture. The solid line is a best fit to the data using

$$
1-e^{2 r^{2} / w^{2}\left(z_{\text {lens }}\right)} \text {. }
$$

For this width, the lens fill factors were 0.63 and 1.63 for the Olympus 20x/0.95W and Nikon $60 \mathrm{x} / 1.0 \mathrm{~W}$, respectively. The minimum focal widths $w^{\prime}{ }_{0}\left(z_{0}\right)$ were determined from best fits to the two-dimensional fluorescence intensity distributions obtained from single planar $\mathrm{x}-\mathrm{y}$ scans using $e^{-2\left(x^{2}+y^{2}\right) / w_{0}^{\prime 2}}$. The $\mathrm{x}-\mathrm{y}$ planar scans were taken at $z$-positions with the highest peak fluorescence intensity. Figure 4.8 shows the measured focal widths versus normalised focus depth $\alpha z_{0}$ for the objectives used. In both cases, the measured focal beam widths are significantly larger than expected; $\sim 56 \%$ for the Olympus $20 \mathrm{x} / 0.95 \mathrm{~W}$ and $\sim 38 \%$ for the Nikon $60 \mathrm{x} / 1.0 \mathrm{~W}$. This discrepancy could be due to several factors. First, although lens aberrations are corrected to less than perceivable limits for visual observation in most objectives, diffraction limited performance is only provided under specific operating conditions. Problematic in our case is the use of near infrared excitation light since the used objectives are designed for wavelengths in the visible. It can thus be expected that the correction for chromatic but also spherical aberrations is not ideal. ${ }^{5}$ Second, high NA objectives are often found to exhibit residual spherical aberration components of very high order, which can 
reduce the nominal NA by up to $10 \% .{ }^{6}$ However, apart from the significant deviation from the absolute values for the focal beam widths it can be seen that the absolute increase in focal width with focus depth i.e. the slope of the curve is well approximated by the predicted course (Eqn. 4.21). This suggests that the aberrations are depth independent providing only a constant offset to the focal beam width and are in great part induced by the used optics and not by the sample.

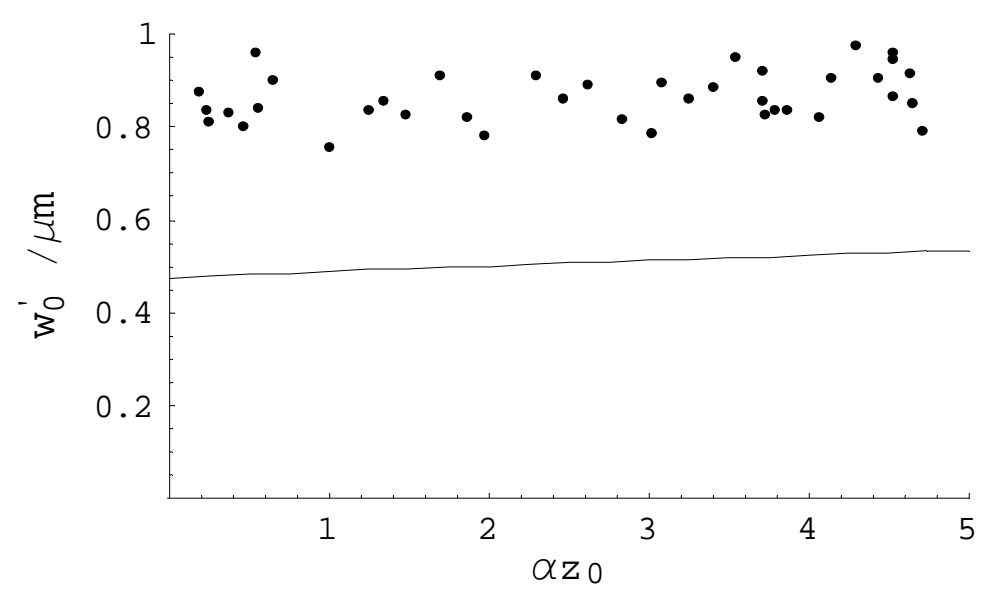

(a)

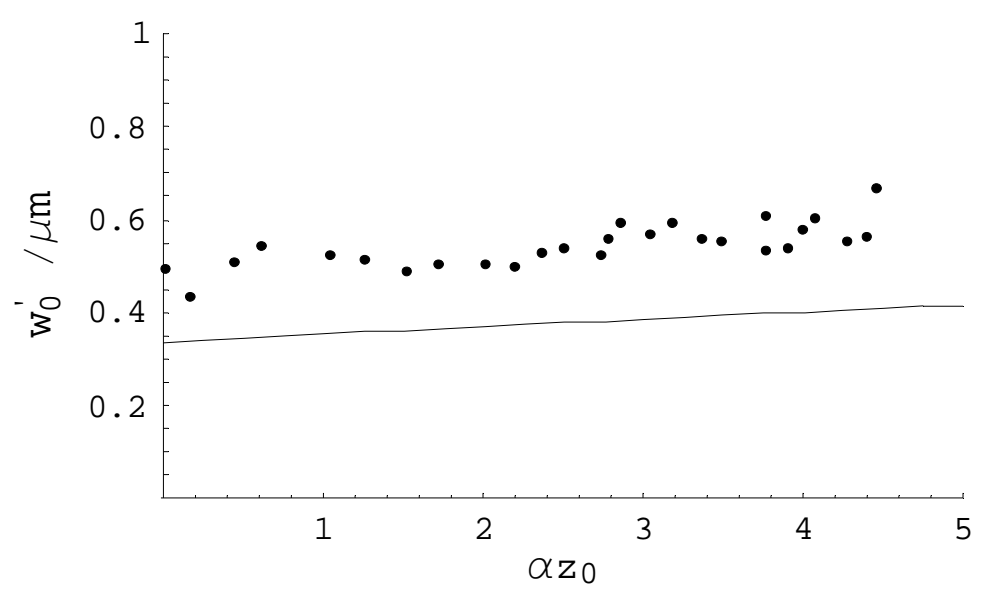

(b)

Fig. 4.8 Beam waist $w_{0}{ }_{0}$ versus normalised focus depth $\alpha z_{0}$ and theoretical course according to Eqn. 4.21 for two different objectives and lens fill factors (a) Olympus XLUMPlanF1 20x/0.95W with $\beta=0.63$ and (b) Nikon Fluor DIC M 60x/1.0W with $\beta=1.63$.

\subsubsection{Focal two-photon fluorescence}

In order to calculate the focal two-photon fluorescence $F_{S}$ for a particular experimental situation, one needs to evaluate Eqn. 4.2. Inserting Eqn. 4.7 into Eqn. 4.2 and assuming a 
constant fluorophore concentration throughout the integrated volume and pulsed incident light with a Gaussian temporal profile and pulse energy $E$, i.e.

$$
P(t)=\sqrt{\frac{2}{\pi}} \frac{E}{\tau_{0}} e^{-\frac{2 t^{2}}{\tau_{0}^{2}}}
$$

which yields

$$
F_{S} \propto \delta C_{S} \iint I^{2}(\rho, z, t) \mathrm{d} t \mathrm{~d} V
$$

The integral in Eqn. 4.24 can be solved analytically if the lateral intensity distribution displays circular symmetry and the lateral extent of the fluorescent volume is much larger than the beam width. In this case, the integration can be performed over the radial co-ordinate and extended to infinity without introducing significant errors i.e.

$$
\int_{Y} I^{2}(\rho, z) \mathrm{d} V \cong \int_{-z^{\prime}}^{z^{\prime}} \int_{0}^{\infty} I^{2}(\rho, z) 2 \pi \rho \mathrm{d} \rho \mathrm{dz}
$$

which, using the intensity distribution in Eqn. 4.7, gives

$$
\begin{aligned}
& \int_{-z^{\prime}}^{z^{\prime}} \int_{0}^{\infty} \int_{-\infty}^{\infty} I^{2}(\rho, z, t) \mathrm{d} t 2 \pi \rho \mathrm{d} \rho \mathrm{dz}= \frac{\delta C_{S} E^{2}}{\tau_{0} \sqrt{\pi}} \frac{2 n^{2} \pi}{\lambda\left(16 n^{2} \pi^{2}+\alpha^{2} \lambda^{2}\right)} e^{-2 \alpha z_{0}} \\
&\left\{\begin{array}{l}
(\alpha \lambda-i 4 n \pi) e^{i 2 \alpha z_{r}}[\operatorname{Ei}(A)-E i(-B)]+c . c . \\
\\
\left.-2 \alpha \lambda e^{8 n \pi z_{r} / \lambda}[\operatorname{Ei}(C)-\operatorname{Ei}(D)]\right\}
\end{array}\right.
\end{aligned}
$$

where

$$
\begin{aligned}
& A=2 \alpha\left(z^{\prime}-i z_{r}\right) \\
& B=2 \alpha\left(z^{\prime}+i z_{r}\right) \\
& C=2 \alpha z^{\prime}-8 n \pi z_{r} / \lambda \\
& D=-2 \alpha z^{\prime}-8 n \pi z_{r} / \lambda
\end{aligned}
$$

and $\operatorname{Ei}(x)$ is the exponential integral function defined as 


$$
E i(x)=\int_{-x}^{\infty} \frac{e^{-x^{\prime}}}{x^{\prime}} \mathrm{d} x^{\prime}
$$

Since in most practical situations $2 \alpha z^{\prime} \ll 8 n \pi z_{r} / \lambda, \quad C \approx D \quad$ and thus $2 \alpha \lambda e^{8 n \pi \pi_{r} / \lambda}[\operatorname{Ei}(C)-E i(D)] \approx 0$ (at least five orders of magnitude smaller than the other terms), Eqn. 4.26 can be simplified to

$$
\begin{aligned}
\int_{-z^{\prime}}^{z^{\prime}} \int_{0}^{\infty} \int_{-\infty}^{\infty} I^{2}(\rho, z, t) \mathrm{d} t 2 \pi \rho \mathrm{d} \rho \mathrm{d} \mathrm{z} \approx & \frac{\delta C_{S} E^{2}}{\tau_{0} \sqrt{\pi}} \frac{2 n^{2} \pi}{\lambda\left(16 n^{2} \pi^{2}+\alpha^{2} \lambda^{2}\right)} e^{-2 \alpha z_{0}} \\
& \left\{(\alpha \lambda-i 4 n \pi) e^{i 2 \alpha z_{r}}[\operatorname{Ei}(A)-\operatorname{Ei}(-B)]+c . c .\right\}
\end{aligned}
$$

For very thick specimens i.e. $z^{\prime} \gg z_{r}$ and very long optical mean-free-path lengths $l=1 / \alpha \rightarrow$ $\infty$ the integral in Eqn. 4.24 can be further simplified to

$$
\left.\int_{-z^{\prime}}^{z^{\prime}} \int_{0}^{\infty} \int_{-\infty}^{\infty} I^{2}(\rho, z, t) \mathrm{d} t 2 \pi \rho \mathrm{d} \rho \mathrm{dz}\right|_{l \rightarrow \infty}=\frac{\delta C_{S} E^{2}}{\tau_{0} \sqrt{\pi}} \frac{n \pi}{\lambda},
$$

which is independent of the numerical aperture of the focussing lens - a peculiar property of two-photon excitation in a focussed beam ${ }^{7}$ which can be explained by the fact that a change in the excitation efficiency due to a change in NA is exactly balanced by the corresponding change in the excitation volume.

In imaging applications, however, one is often interested in fluorophore filled volumes smaller than those assumed for the derivation of Eqns. 4.28 and 4.29, and Eqn. 4.24 has to be evaluated numerically.

For typical conditions encountered in two-photon imaging of brain tissue i.e. $l \approx l_{s}=200 \mu \mathrm{m}$, $n=1.33, \lambda=0.9 \mu \mathrm{m}$ we have evaluated the focal fluorescence generation for two different fluorescent-volume geometries, for spherical volumes, by solving Eqn. 4.24 numerically, and for fluorescent sheets, using Eqn. 4.28. In Fig. 4.9 we have plotted the relative fluorescence for (a) a spherical fluorescent volume as a function of sphere radius and (b) a fluorescent sheet with an area $A \rightarrow \infty$ as a function of sheet thickness for various effective NA's. 


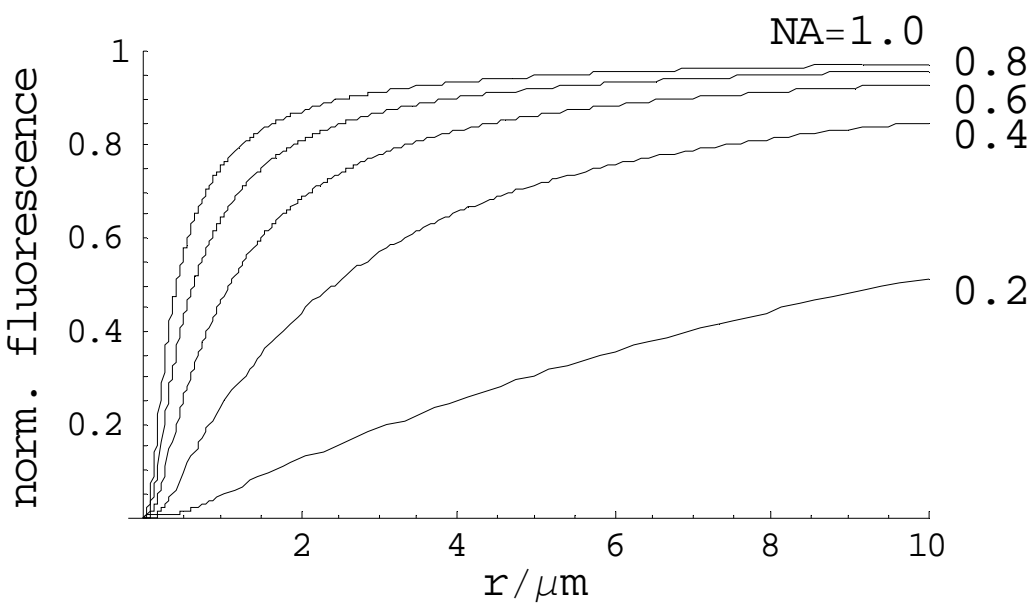

(a)

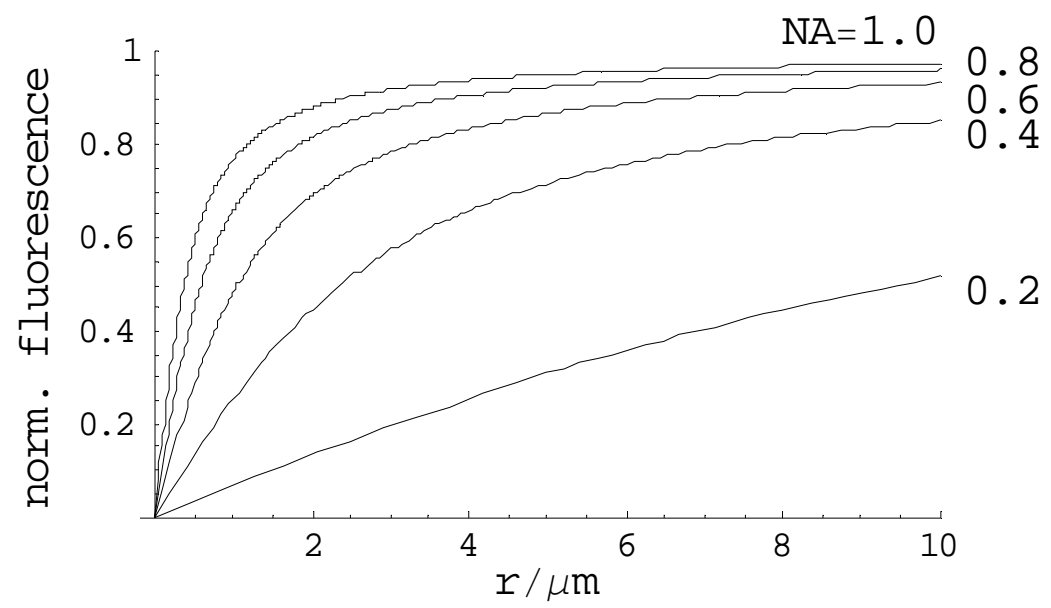

(b)

Fig. 4.9 Normalised focal fluorescence $F\left(V=4 / 3 \pi r^{3}\right) / F(V \rightarrow \infty)$ as a function of radius for a spherical fluorescent volume (a) and $F\left(V=d^{*} A, A \rightarrow \infty\right) / F(V \rightarrow \infty)$ as a function of thickness of a fluorescent sheet (b) for various effective NA's. Note that, for better comparison, both graphs have been plotted as a function of $r$ which in (a) specifies the sphere radius and in (b) half the sheet thickness ( $r=d / 2)$.

Differences in the focal fluorescence for the two fluorescent geometries become significant only for small $r$ i.e. for structures smaller than the beam's minimal waist. In fact, for $r \gtrsim 1.3 w_{0}$ the relative difference $\left[1-F\left(V=4 / 3 \pi r^{3}\right) / F(V=2 r A, A \rightarrow \infty)\right] \leq 10 \%$ (see Fig. 4.10). 


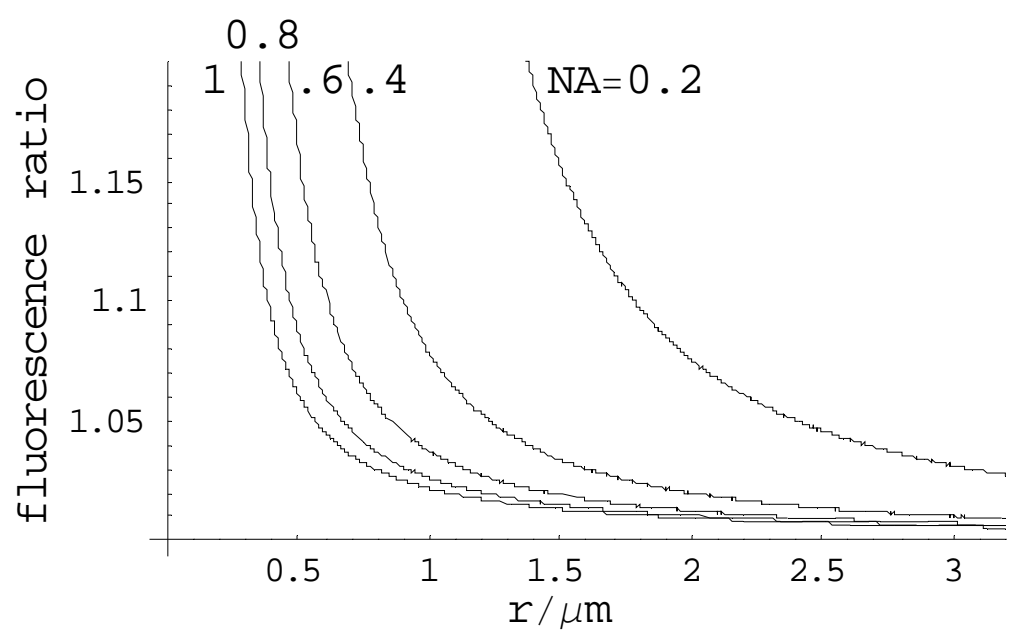

Fig. 4.10 Ratio of focal fluorescence of spherical and sheet fluorescent volumes $F(V=2 r A, A \rightarrow \infty) / F(V=$ $\left.4 / 3 \pi r^{3}\right) v s . r$.

However, Fig. 4.9 also shows that for fluorescent objects up to a size of many $w_{0}$, the generated fluorescence depends strongly on the effective NA. Since this is the case for the vast majority of applications in two-photon microscopy, increases in the excitation efficiency and thus imaging depth can be achieved by using objectives with the highest possible NA. To illustrate this, we have plotted the relative fluorescence $F(N A, r) / F(N A, r \rightarrow \infty)$ obtained from spherical objects of various radii as a function of effective NA (see figure 4.11).

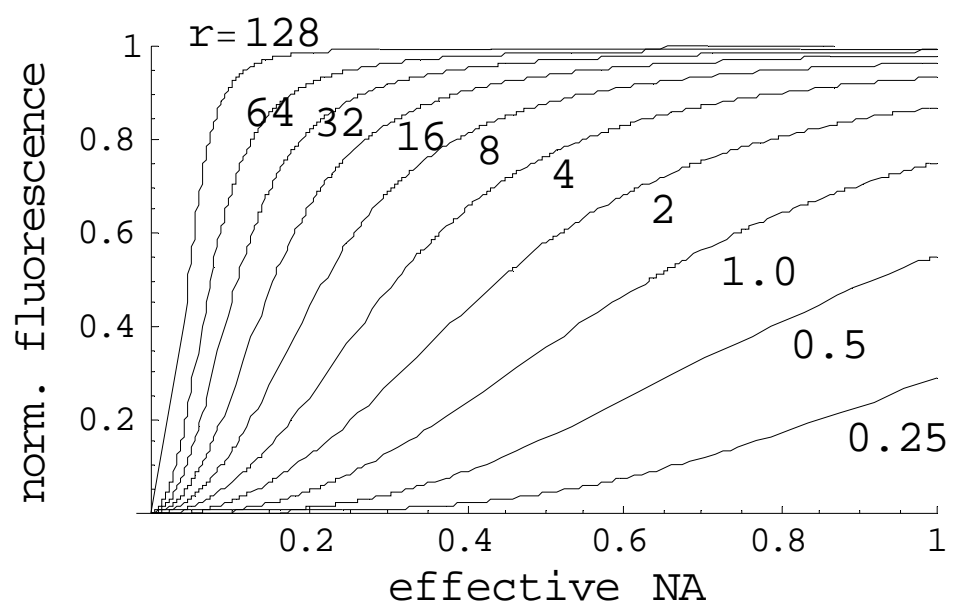

Fig. 4.11 Relative fluorescence $F(N A, r) / F(N A, r \rightarrow \infty) v s$. effective NA for spherical fluorescent volumes with radii $\rightarrow r=0.25,0.5,1,2,4,8,16,32,64,128 \mu \mathrm{m}$. 


\subsubsection{Scattered excitation light}

It is widely believed that two-photon fluorescence is largely confined to the vicinity of the focus formed by the ballistic light. While for the majority of applications in two-photon microscopy this is a reasonable assumption, at large imaging depths it is no longer true. This is because in order to maintain a constant excitation power in the focus, the incident power needs to be increased exponentially with depth. This exponential increase, although partially compensated by the increase of the beam cross-section at the surface, leads at large depths to a steep increase in excitation intensity at and near the surface and thus to an increase in out-offocus fluorescence generation. In specimens that are strongly forward scattering, this includes most biological tissues, ${ }^{8}$ the assumption that scattered light is generally much diluted compared to ballistic light no longer holds. In particular near the surface, the majority of scattered light will remain within the ballistic excitation beam and will therefore be equally likely to generate two-photon fluorescence as long as the scattered light has not sufficiently dispersed temporally.

For the calculation of the two-photon fluorescence it is, therefore, important to include both ballistic and scattered contributions. While the ballistic contribution can be determined quite accurately (see previous sections), calculation of the scattered contribution is difficult.

The propagation of pulsed laser radiation through turbid media with highly anisotropic scattering has been investigated using several different approaches including radiative transfer models $^{9,} 10$ and Monte Carlo techniques ${ }^{11}$. Recently, analytical methods using simple statistical concepts were developed to treat scattering in turbid water. ${ }^{12,}{ }^{13}$ Although their validity for treating biological samples still needs to be verified, these models provide a first approximation for the temporal and spatial spread of multiply scattered light.

Following the approach by McLean et al. ${ }^{13}$, the variance of the transverse spread $\left(\sigma_{r}^{2}\right)$ and the variance of the temporal spread $\left(\sigma_{l}^{2}\right)$ for a pulsed unidirectional beam incident on a medium with highly anisotropic scattering are, using the small-angle approximation, given by

$$
\sigma_{r}^{2}(z)=\frac{1}{3} \frac{z^{3}}{l_{s}}\left\langle\Theta^{2}\right\rangle
$$

and

$$
\sigma_{\tau}^{2}(z)=\frac{1}{12} \frac{z^{3}}{c^{2} l_{s}}\left\langle\Theta^{4}\right\rangle+\frac{1}{24} \frac{z^{4}}{c^{2} l_{s}^{2}}\left\langle\Theta^{2}\right\rangle^{2}
$$


respectively, with $\Theta=2 \sin (\theta / 2)$ and $\theta$ the scattering angle. The statistical averages, i.e. the moments, of $\Theta$,

$$
\begin{gathered}
\left\langle\Theta^{2}\right\rangle=\left\langle 4 \sin ^{2}(\theta / 2)\right\rangle=\langle 2-2 \cos (\theta)\rangle=2-2\langle\cos (\theta)\rangle, \\
\left\langle\Theta^{4}\right\rangle=\left\langle 4-8 \cos (\theta)+4 \cos ^{2}(\theta)\right\rangle=4-8\langle\cos (\theta)\rangle+4\left\langle\cos ^{2}(\theta)\right\rangle,
\end{gathered}
$$

can be calculated using ${ }^{14}$

$$
\left\langle x^{n}\right\rangle=\frac{1}{2} \int_{-1}^{1} x^{n} p(x) d x
$$

where $x=\cos (\theta)$ and $p(x)$ is the scattering phase function. Calculation of the scattering phase function in media containing a large variety of scatterers is difficult and it is often necessary to revert to approximations. An approximate scattering phase function commonly used in biological tissue optics is the Henyey-Greenstein scattering phase function ${ }^{15}$

$$
p_{H G}(x)=\frac{1-g^{2}}{\left(1+g^{2}-2 g x\right)^{3 / 2}},
$$

where

$$
g=\langle\cos (\theta)\rangle=\frac{1}{2} \int_{-1}^{1} x p_{H G}(x) d x
$$

and

$$
\frac{1}{2} \int_{-1}^{1} p_{H G}(x) d x=1
$$

Using Eqn. 4.34 the second moment is given by

$$
\left\langle\cos ^{2}(\theta)\right\rangle=\frac{1}{2} \int_{-1}^{1} x^{2} p_{H G}(x) d x=\frac{1}{3}\left(1+2 g^{2}\right) .
$$

Thus

$$
\left\langle\Theta^{2}\right\rangle=2(1-g) ; \quad\left\langle\Theta^{4}\right\rangle=8 / 3(g-2)(g-1)
$$


Hence Eqns. 4.30 and 4.31 reduce to

$$
\sigma_{r}^{2}(z)=\frac{2}{3} \frac{z^{3}}{l_{s}}(1-g),
$$

and

$$
\sigma_{\tau}^{2}(z)=\frac{2}{9} \frac{z^{3}}{c^{2} l_{s}}(g-2)(g-1)+\frac{1}{6} \frac{z^{4}}{c^{2} l_{s}^{2}}(1-g)^{2}
$$

In line with the statistical approach used to derive Eqns. 4.40 and 4.41, a Gaussian beam can be viewed as being made up of a fan of rays travelling at various angles to the optical axis towards a common focus. In this, ray optical, approximation the effective temporal and transverse spatial beam widths are

$$
\tau_{e f f}(z)=\sqrt{\tau_{0}^{2}+4 \sigma_{\tau}^{2}(z)}
$$

and

$$
w_{e f f}(z)=\sqrt{w\left(z-z_{0}\right)^{2}+2 \sigma_{r}^{2}(z)}
$$

where $\tau_{0}$ and $w\left(z-z_{0}\right)$ are the $1 / \mathrm{e}^{2}$ temporal and lateral widths of a pulsed Gaussian beam in free space with its focus centred at $z=z_{0}$ respectively (the temporal FWHM is larger by a factor $\sqrt{-2 \ln [0.5]} \approx 1.18$ ).

Considering ballistic and scattered light and assuming, as is appropriate for most biological tissues, that scattering is dominant and absorption negligible, the beam intensity will, with depth, only be reduced by scattering i.e. by temporal and spatial broadening of the light distribution. Assuming that the variances given in Eqn. 4.30 and 4.31 pertain to a Gaussian distribution, the intensity of the scattered light can be written as:

$$
I_{\text {scat. }}(\rho, z, t)=\frac{2 P_{\text {scat. }}(t, z)}{\pi w_{\text {eff }}^{2}(z)} e^{-\frac{2 \rho^{2}}{w_{e f f}^{2}(z)}}
$$

with (considering a single pulse of energy $E$ )

$$
P_{\text {scat. }}(t, z)=\sqrt{\frac{2}{\pi}} \frac{E}{\tau_{e f f}(z)} e^{\frac{2 t^{2}}{\tau_{e f f}^{2}(z)}}\left(1-e^{-\alpha z}\right) .
$$




\subsubsection{Out-of-focus two-photon fluorescence}

The out-of-focus fluorescence is proportional to the integral of $\left(I_{\text {scat. }}(\rho, z, t)+I_{\text {ball. }}(\rho, z, t)\right)^{2}$ over the out-of-focus volume $V_{\text {oof }}$ and time:

$$
F_{B} \propto \delta C_{B} \int_{V_{\text {oof }}} \int_{-\infty}^{\infty}\left(I_{\text {scat. }}(\rho, z, t)+I_{\text {ball. }}(\rho, z, t)\right)^{2} \mathrm{~d} t \mathrm{~d} V .
$$

We have evaluated Eqn. 4.46 numerically for the experimental parameters $l_{s}=200 \mu \mathrm{m}, n=$ 1.33, $\lambda=0.9 \mu \mathrm{m}$, effective $N A=0.6, g=0.9$ and $\tau_{0}=100$ fs. Figure 4.12 shows the fluorescence integrated over transversal planes versus depth.

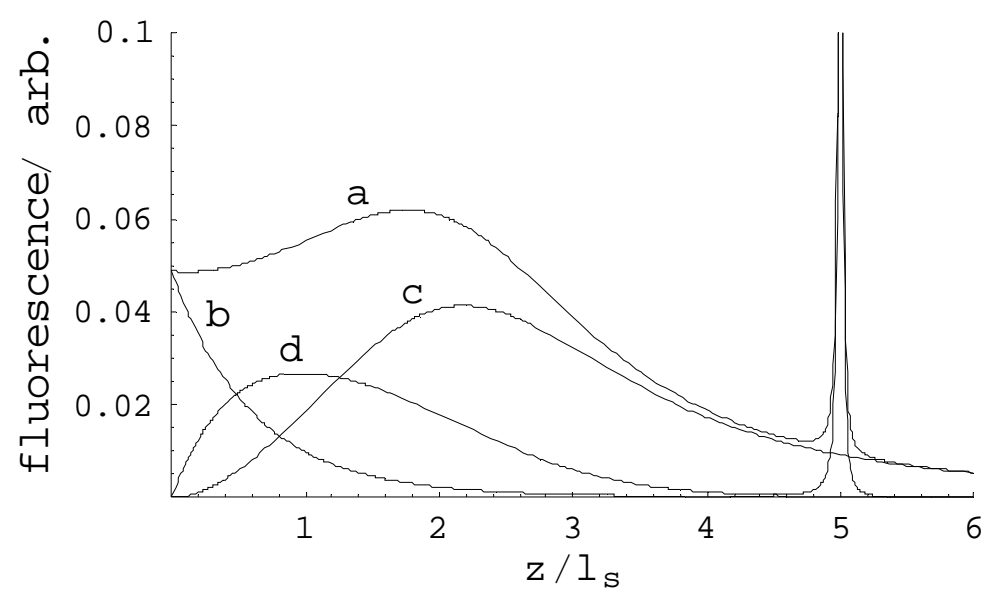

Fig. 4.12 Radially integrated fluorescence $v s$. depth for (a) the total fluorescence proportional to the squared sum of scattered and ballistic light intensities $\left(I_{\text {scat. }}(\rho, z, t)+I_{\text {ball. }}(\rho, z, t)\right)^{2},(\mathrm{~b})$ the ballistic light $\left(I_{\text {ball. }}(\rho, z, t)\right)^{2},(\mathrm{c})$ the scattered light $\left(I_{\text {scat. }}(\rho, z, t)\right)^{2}$, and $(\mathrm{d})$ the mixed term $2 I_{\text {scat. }}(\rho, z, t) I_{\text {ball. }}(\rho, z, t)$. The focus depth is $z_{0}=5 l_{\mathrm{s}}=$ $1000 \mu \mathrm{m}$, and the maximum fluorescence intensity in the focus is 1 in arbitrary units.

While for the ballistic fraction, the contribution to the out-of-focus fluorescence is highest at the surface, the scattered fraction and the mixed term have their peak at a depth well below the surface. For low anisotropy factors, this peak will be closer to the surface (this finding is in line with experimental results ${ }^{16}$ ), which can be explained by the fact that significant amounts of scattered light are present only at depths beyond one scattering mean-free-path length. In addition, since most scattered light initially remains within the converging excitation cone, the excitation intensity actually increases with depth up to the point where the reduction due to the spatial and temporal spread begins to dominate. This, of course, occurs closer to the surface if the temporal and spatial width of the incident light are smaller. This 
suggests, that in order to minimise the amount of out-of-focus fluorescence one should use the shortest pulse widths and the smallest NA. However, using a small NA reduces the excitation efficiency in the focus to a much larger extent than in the out-of-focus region so that this is not an option.

\subsection{Two-photon fluorescence detection}

As mentioned above, two-photon excitation is, in most situations, confined to the focal region and out-of-focus fluorescence is negligible. Hence all fluorescence light emerging from the sample contributes useful signal and as much as possible should be detected. However, for choosing the optimal detection design, the fluorescence-light distribution at the sample surface needs to be known. In general, one can distinguish two limiting cases. First, imaging in transparent media or scattering samples at depths much smaller than their scattering meanfree-path length $l_{s}$. In this (the ballistic) case, most fluorescence photons emerge unscattered and the collection efficiency $\varepsilon_{b}$ (isotropic emission assumed) scales with the solid angle of the objective lens

$$
\varepsilon_{b}=1 / 2\left[1-\cos \left(\theta_{N A}\right)\right]
$$

where $\theta_{N A}$ is the half-angle of the objective's angular aperture. A useful approximation of Eqn. 4.47 for low to moderate NA's is given by

$$
\varepsilon_{b} \approx \frac{1}{\pi}\left(\frac{N A}{n}\right)^{2} .
$$

The factor $1 / 2$ in Eqn. (4.49) is due to the fact that in the standard collection scheme (epifluorescence-collection), light of only one hemisphere is collected. In certain situations (imaging of thin samples), light emerging from the far side of the sample can also be collected by using a the condenser path. Used purely for detection, the refractive index does not need to be matched, which allows the use of high NA oil condensers. In this case, a more than twofold increase in total collected signal can be expected. ${ }^{17}$ For the second case - imaging at depths $z_{0}$ beyond $l_{s}$ - most of the fluorescence light reaching the objective entrance aperture will have been scattered resulting in a spread of its spatial and angular distribution i.e. it seems to originate from an extended source in the focal plane. Whether light is collected or 
not then depends no longer solely on its direction but also on its position at the time it leaves the sample. For intermediate imaging depths where photons experience only a few scattering events (semi-ballistic case), calculations of fluorescence-light distributions are difficult and rely mainly on numerical simulations (Monte Carlo studies). At the limit of large imaging depths $\left(z_{0} \gg l_{s}\right)$, photons generated at the focus experience a large number of scattering events and emerge with a roughly isotropic angular distribution (diffuse case). In this case photons may be viewed as performing a random walk (isotropic scattering) starting from a point source deep within a semi-infinite medium until they reach the surface, escape and do not return. For this geometry, the photon density $\left(G_{F}\right)$ within the medium can be calculated using the method of images ${ }^{18}$ i.e. superposing a virtual point sink outside the medium symmetrically to the surface boundary, and is given by

$$
G_{F}(z, r)=\frac{Q}{4 \pi D}\left(\frac{1}{\sqrt{r^{2}+\left(z_{0}-z\right)^{2}}}-\frac{1}{\sqrt{r^{2}+\left(z_{0}-z\right)^{2}}}\right)
$$

where $Q$ is the fluorescence emission rate, $D=c l_{s} / 3$ is the effective photon diffusion coefficient, and $r$ and $z$ the distances from the optical axes and surface, respectively. The fluorescence light intensity at the surface corresponds to the photon flux $\left(F_{F}\right)$ through the surface and is given by the spatial gradient of the photon density:

$$
F_{F}(r)=\left.D \frac{\partial}{\partial z} G_{F}\right|_{z=0}=\frac{Q}{2 \pi} \frac{z_{0}}{\left(r^{2}+z_{0}^{2}\right)^{3 / 2}}
$$

Only light that escapes within a maximum radius $r_{\max }$ and half-angle $\theta_{\max }$, given by the effective field-of-view radius and angular acceptance of the detection system respectively, can be collected. Note that $r_{\max }$ and $\theta_{\max }$ are not independent and change with imaging depth. The collection efficiency in the diffuse case $\varepsilon_{d}$ is therefore given by the fraction of photons that emerge within an effective field-of-view radius given by the integral of Eqn. (4.50) multiplied by the fraction permitted by the effective angular acceptance of the detection system ${ }^{19}$

$$
\varepsilon_{d}=\left[1-\cos \left(\theta_{\max }\right)\right]\left[1-\frac{z_{0}}{\sqrt{r_{\max }^{2}+z_{0}^{2}}}\right] \text {. }
$$


For depths much larger than the field of view $\left(z_{0} \gg r_{\max }\right)$ the detection efficiency scales as $1 / z_{0}^{2}$. In evaluating Eqn. (4.51) it becomes clear that in order to maximize the collection efficiency of focal fluorescence for deep imaging a large field-of-view is just as important as a high angular acceptance. The use of low magnification, high NA objectives is therefore of paramount importance in two-photon microscopy. In fact, it has been shown, that using a special low magnification, high NA objective (Olympus XLUMPlanFl 20x/0.95W), the detection efficiency can be increased by a factor of 10 compared to a standard $60 \mathrm{x} / 0.9 \mathrm{NA}$ objective. However, in order to maximize the benefit, the entire detection path has to be adapted accordingly. This usually requires large clear-aperture optics and large-field detectors with an appropriate acceptance angle.

While Eqn. 4.47 and 4.51 provide exact descriptions of the fluorescence detection efficiency for the cases of ballistic and diffuse fluorescence, respectively, most applications will contain a mixture of both. While such semi-ballistic/semi-diffuse situations have been investigated using Monte Carlo techniques, ${ }^{19}$ they have not been verified by measurements.

In order to provide some more insights into this subject, we have measured the fluorescence detection efficiency in a) a transparent medium (water) and b) a brain tissue phantom as described in section 3.4.2. For the measurements we used a single $90 \mu \mathrm{m}$ yellow-green (490/515) fluorescent polystyrene micro sphere (Polyscience, Inc.) as the fluorescence source. The sphere was glued to the tip of a $0.22 \mathrm{NA} 105 \mu \mathrm{m}$ core multimode optical fiber (Thorlabs Inc.) used to deliver blue (446 nm) excitation light from a mercury lamp (HBO 50, Carl Zeiss). The optical fibre was mounted on an $x-y-z$ stage to allow free positioning with respect to the objective used(Olympus XLUMPlanFl 20x/0.95W). Fluorescence was separated with a dichroic mirror, focussed with an $18 \mathrm{~mm}$ focal length condensor (diameter: $22.4 \mathrm{~mm}$ ) and detected with a photomultiplier (R3896, Hamamatsu). In order to collect all the fluorescence light emerging from the back aperture of the objective, the photomultiplier was placed as close to the objective as possible (distance between objective back aperture and photomultiplier photocathode $<30 \mathrm{~mm}$ ).

In the first measurement, the fibre tip was positioned in a water tank in front of the immersed objective and the $x-y-z-p o s i t i o n$ control of the microscope head was used to center the fluorescent sphere on the microscope optical axis and focal plane by maximising the detected fluorescence signal. The fluorescence signal was measured by acquiring a z-scan at steps of 
$20 \mu \mathrm{m}$ moving the focus of the objective from $2000 \mu \mathrm{m}$ below (corresponding to the working distance $w . d$. of the objective used) to $2500 \mu \mathrm{m}$ above the fluorescent sphere (see Fig. 4.13).

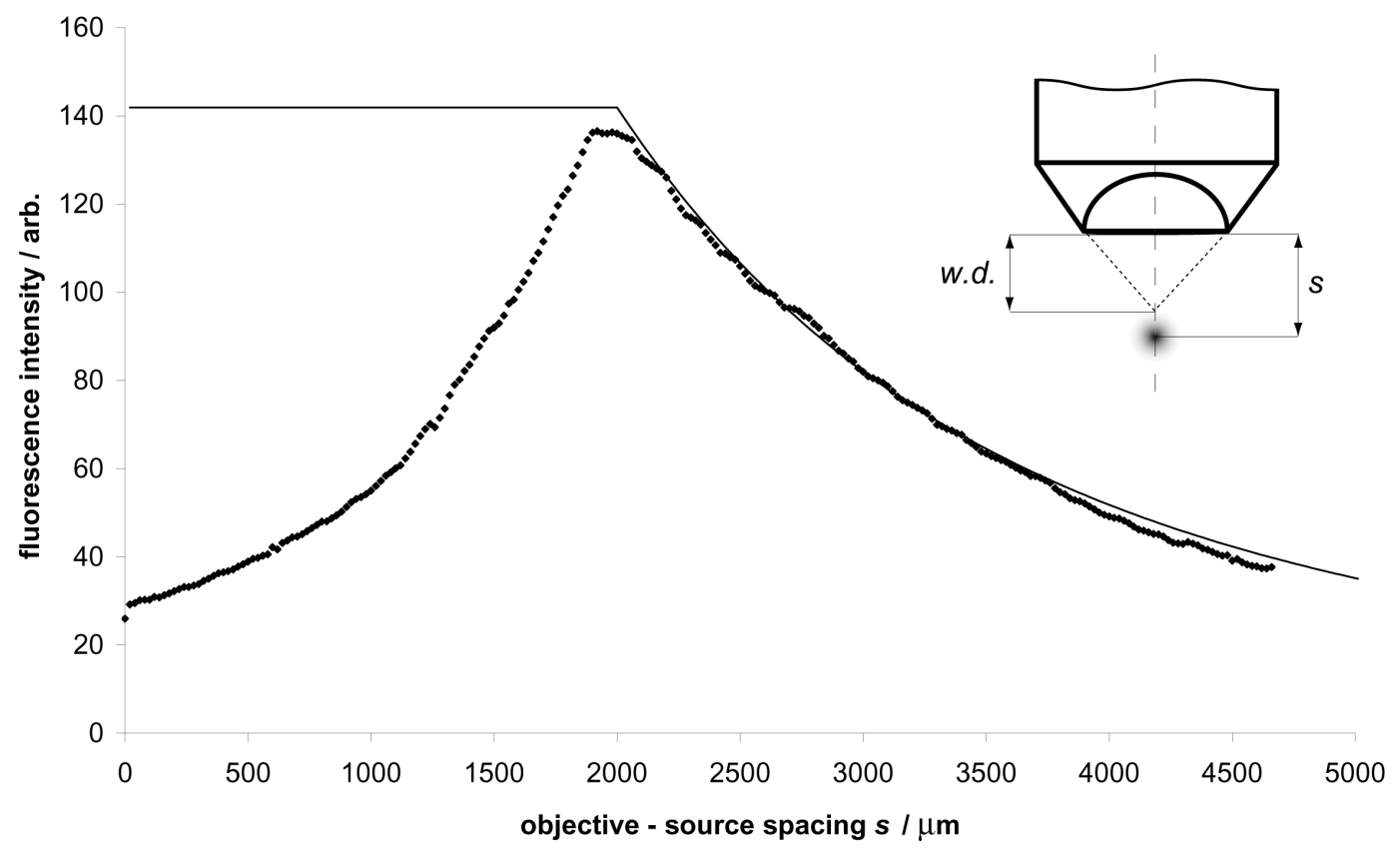

Fig. 4.13 Detected fluorescence intensity from a fluorescent point source vs. objective-source spacing as defined in the inset. The source was embedded in water and centred on the optical axis. The working distance (w.d.) of the objective used (Olympus XLUMPlanFl 20x/0.95W) is $2000 \mu \mathrm{m}$. Solid line is a best fit to $I_{0}\left[1-\cos \left(\theta_{\max }\right)\right]$ where $\theta_{\max }=\arctan \left(R_{\text {lens }} / s\right)$ and $R_{\text {lens }}=w \cdot d \cdot \tan \left(\theta_{N A}\right)=2041 \mu \mathrm{m}$ is the open aperture radius of the objective front lens.

As can be seen from Fig. 4.13, for $s>$ w.d. the detected fluorescence intensity closely follows the predicted course, which indicates that with the set-up used all the fluorescence incident on the front lens of the objective is collected. For objective-source spacings smaller than the working distance, the detection efficiency decreases steeply. This is in contrast to the behaviour predicted by Eqn. $4.47^{\dagger}$. However, since Eqn. 4.47 is based on geometrical considerations only, it neglects, for example, that light travelling at angles greater than about 65 degrees is totally reflected at the water glass interface. More important however is that objectives are not likely to accommodate for angles larger than those defined by their nominal NA. But even if one would restrict the angle spectrum of the point source to that given by the objective's NA, for source positions in between working distance and lens surface i.e. $s<$ $w . d$., light will be clipped by internal structures and apertures of the objective.

\footnotetext{
${ }^{\dagger}$ exchanging $\theta_{N A}$ with $\theta_{\max }=\arctan \left[R_{\text {lens }} / s\right]$
} 
Next we measured the fluorescence detection efficiency in the brain tissue phantom for different depths of the fluorescent sphere. In order to minimise damage to the phantom containing only non-fluorescent scattering beads (see section 3.4.2), changing the depth position of the fluorescent sphere was done by moving the fibre along its axis. For this, the $\mathrm{x}-$ $\mathrm{y}-\mathrm{z}$ stage holding the fibre tip was tilted with respect to the horizontal by about 11 degrees. Since the position of the fluorescent sphere within the phantom can not be tracked, centring the bead on the optical axis by retracing the objective to the point of maximum fluorescence signal as in transparent media is not possible. Therefore, the trajectory of the fluorescent sphere needed to be calibrated. For this we used a transparent agarose phantom, which we assumed to have comparable mechanical properties. For several successive fibre feeds of 500 $\mu \mathrm{m}$ we measured the $x, y$ and $z$ co-ordinates by retracing the objective to the position of maximum fluorescence signal and found the average angle to the horizontal to be 12.25 degrees corresponding to a fibre advance of $471 \mu \mathrm{m}$ for a required depth increase of $100 \mu \mathrm{m}$. In this measurement, the depth of the fluorescence sphere in the phantom $\left(z_{0}\right)$ was changed from 0 to $1100 \mu \mathrm{m}$ in steps of $100 \mu \mathrm{m}$, and the objective retracted accordingly. At each depth setting, the fluorescence was measured by acquiring a $z$-scan for focus settings from $1100 \mu \mathrm{m}$ below to $2000 \mu \mathrm{m}$ above the phantom surface (see Fig. 4.14).

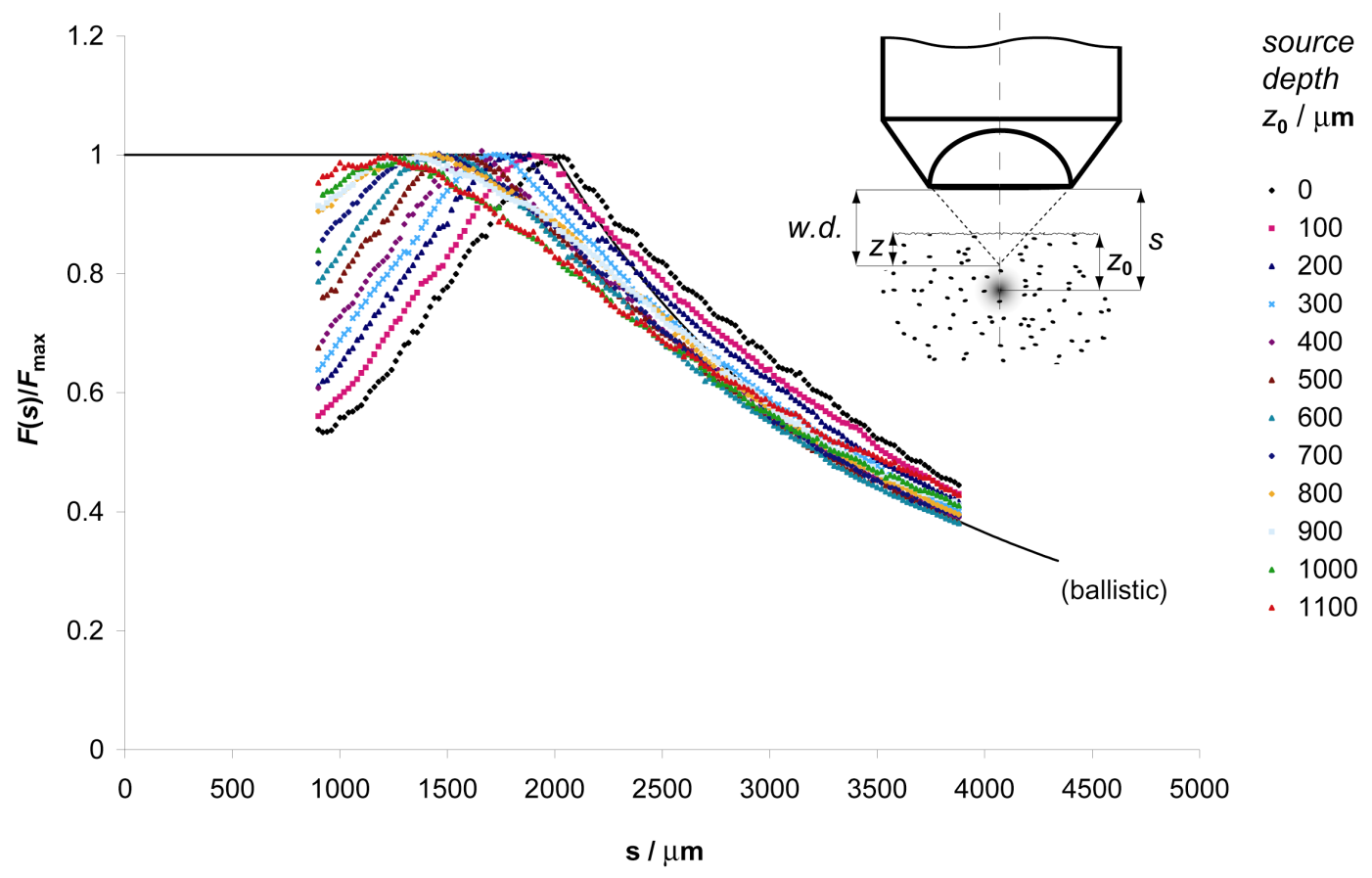

Fig. $4.14 \quad$ Normalised detected fluorescence intensity from a fluorescent point source for various source depths $\left(z_{0}\right)$ in a brain tissue phantom $v s$. objective source spacing $(s)$ as defined in the inset. The working distance (w.d.) of the objective used (Olympus XLUMPlanFl 20x/0.95W) is $2000 \mu \mathrm{m}$ and the fluorescent point source was always centred on the objective's optical axis. The solid line corresponds to the ballistic case. 
Several interesting characteristics regarding the fluorescence detection efficiency can be deduced from the acquired data presented in Fig. 4.14. First, while for small source depths, the fluorescence maximum closely matches the source's depth position, for source depths beyond about $300 \mu \mathrm{m}$, corresponding to 4 scattering mean-free-path lengths $\left(l_{s}=77 \mu \mathrm{m}\right.$ at a wavelength of $515 \mathrm{~nm}$ ) the fluorescence maximum shifts closer to the surface at a rate of about $0.4 z_{0}$ (see figure 4.15). This can be explained by the fact that fluorescence light reaching the objective entrance aperture will have been scattered resulting in a spread of its spatial and angular distribution i.e. it appears to originate from an extended source in the focal plane. In such situations, the collection efficiency can be increased by increasing the objective-source spacing i.e. the objective's effective field-of-view at the source plane. However, since increasing the objective-source spacing also decreases the effective collection solid angle, the increase in the collection efficiency is limited to the point where the latter becomes dominant. Apart from this limitation such an approach is generally not feasible in two-photon microscopy since it requires decollimation of the excitation beam incident on the back aperture of the objective to shift the excitation focal volume away from its nominal position. This comes at the cost of increased optical aberrations and thus reduced excitation efficiency.

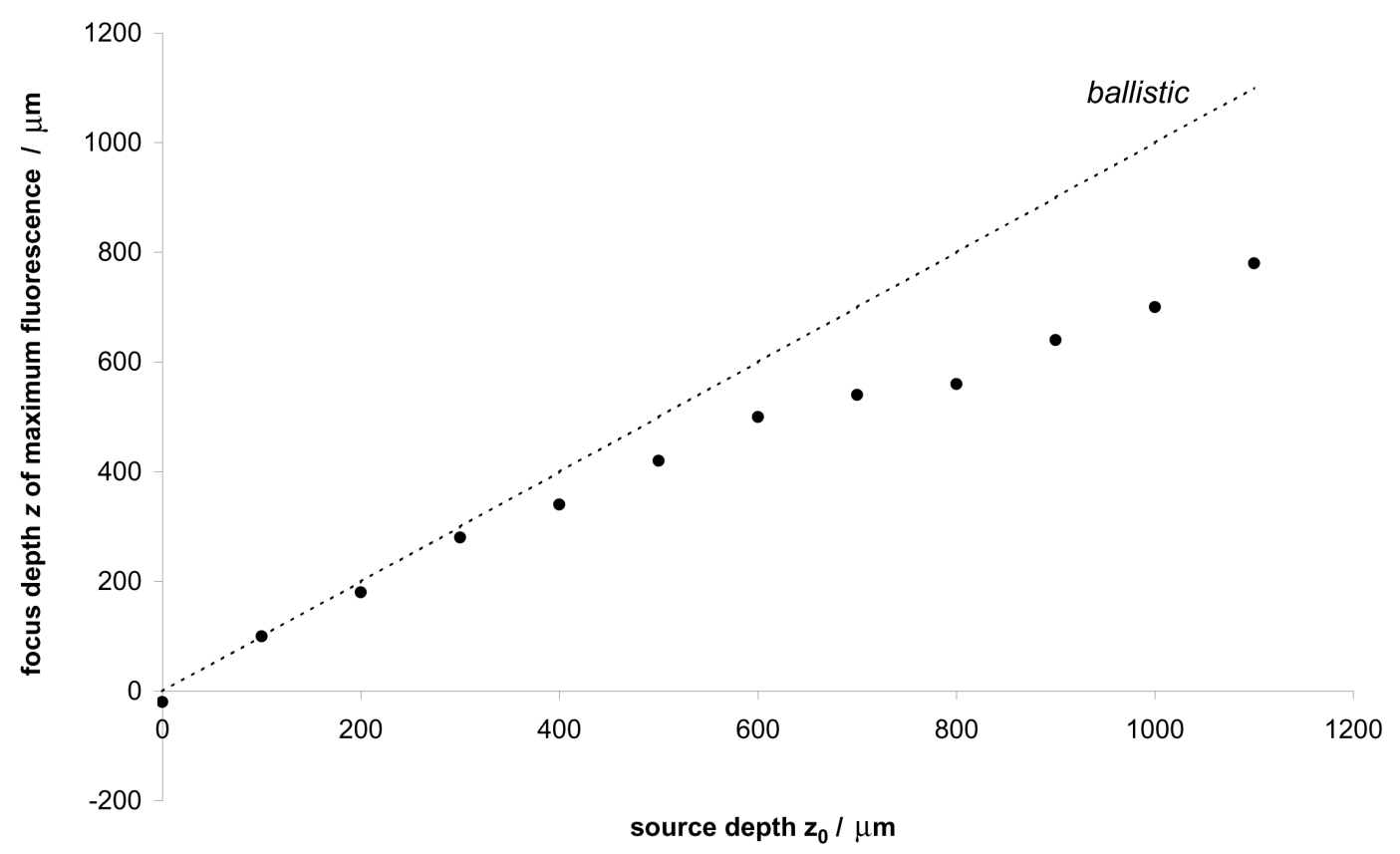

Fig. 4.15 Focus depth $z$ of fluorescence maximum $v$ s. source depth $z_{0}$. Dashed line corresponds to the ballistic case. 
Second, in situations where $s \leq w . d$. i.e. the source is located between the objective's focal plane and the phantom surface, the detection efficiency scales roughly linearly with source depth (at a rate of about $0.24 \alpha z_{0}$ ) for source depths up to about $800 \mu \mathrm{m}$ or $\sim 10$ scattering mean-free-path lengths (see Fig. 4.16). This finding is of particular importance since it provides a measure for the detection efficiency of out-of-focus fluorescence (contributions from the far side of the focus are small and can usually be neglected).

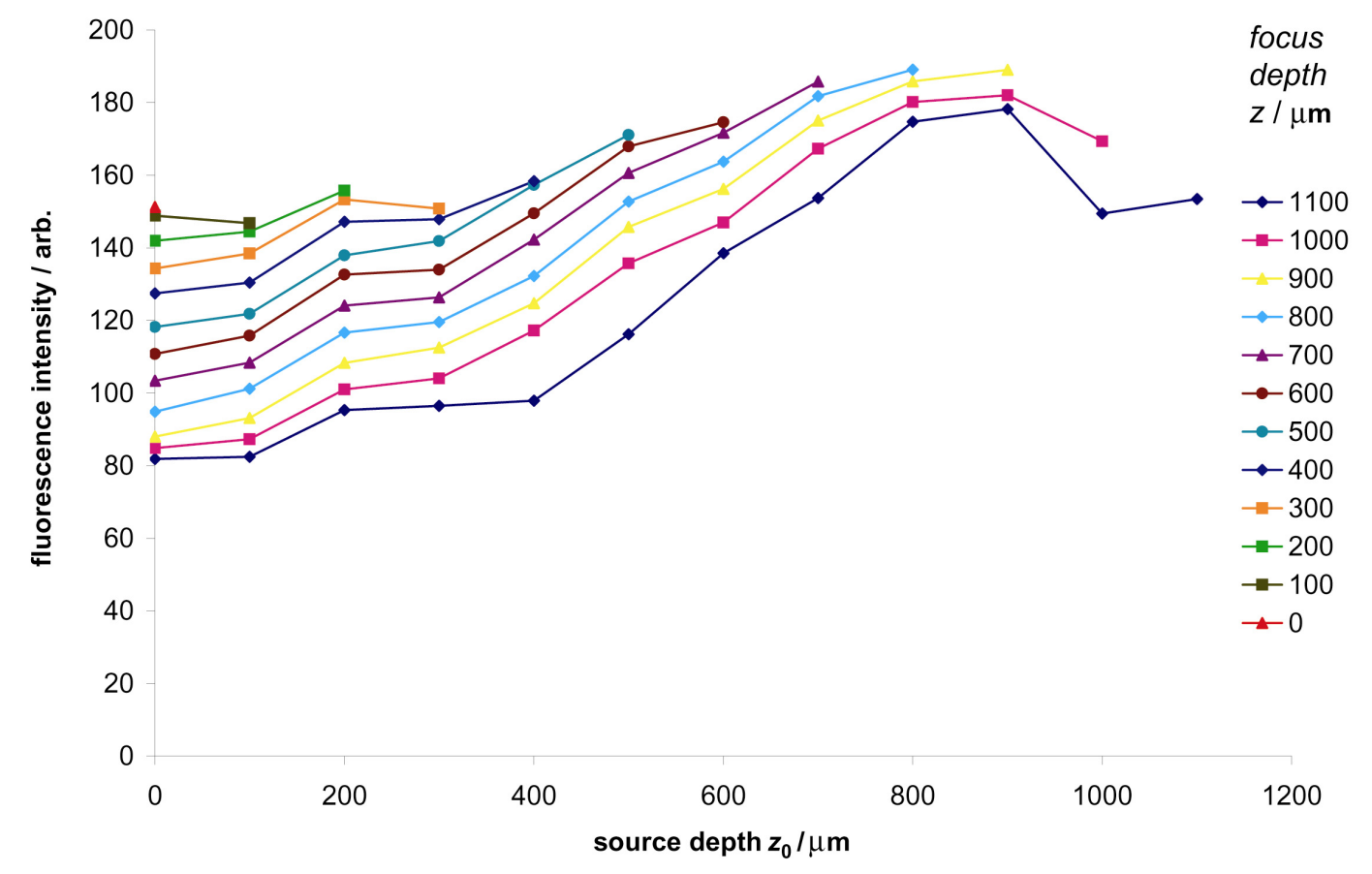

Fig. 4.16 Total detected fluorescence vs. source depth $z_{0}$ for various focus depths $z$.

Third, a measure for the detection efficiency for the focal fluorescence, can be inferred from the data points $F\left(z=z_{0}\right)$ corresponding to the upper envelope of the traces in Fig. 4.16. For a better illustration, we have plotted these data in terms of normalised detection efficiency $F(z=$ $\left.z_{0}\right) / F_{\text {ball. }}$ where $F_{\text {ball. }}$ is the fluorescence detected in the non-scattering medium i.e. ballistic case (see Fig. 4.17). 


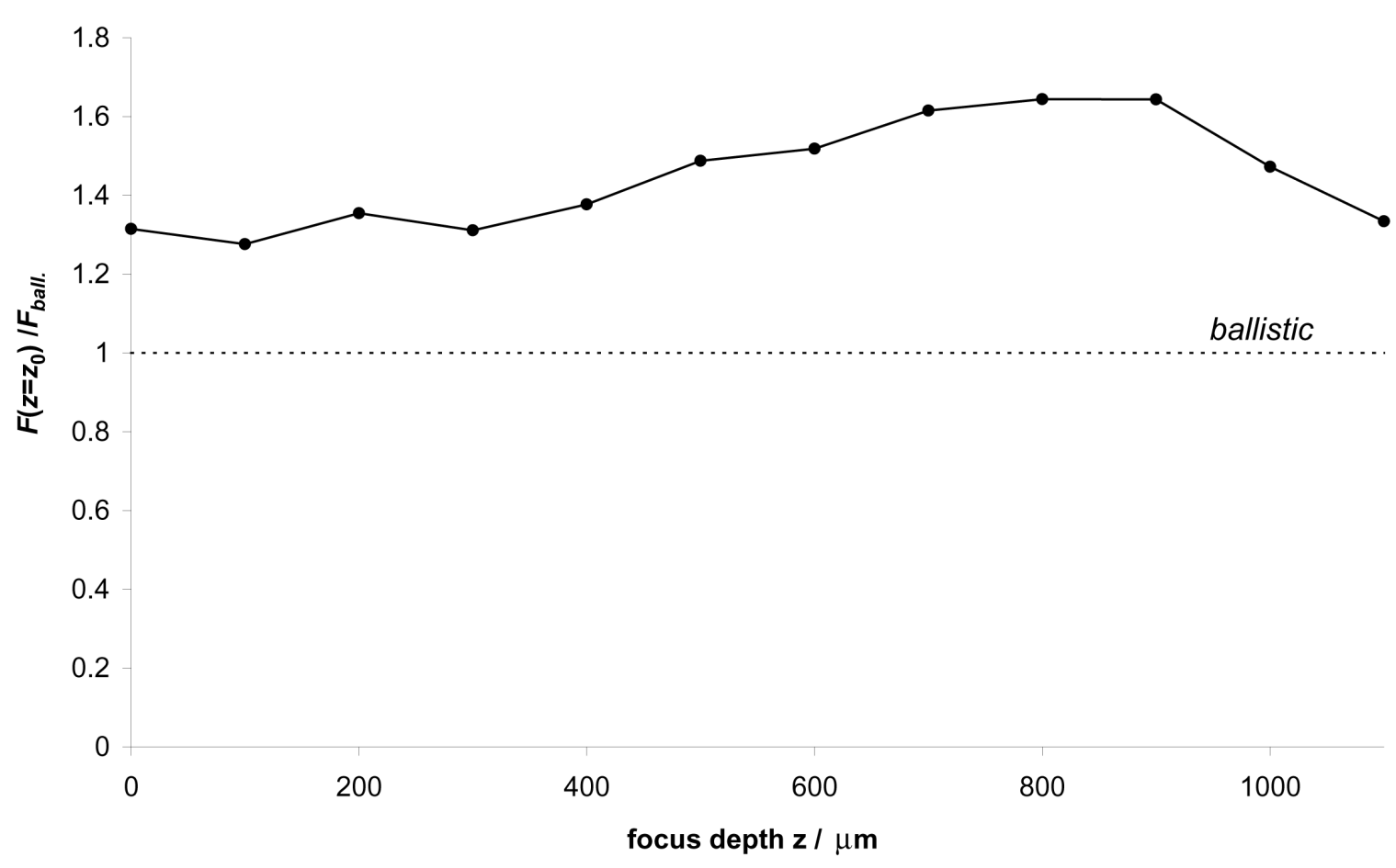

Fig. 4.17 Normalised detection efficiency $F\left(z=z_{0}\right) / F_{\text {ball }} v s$. focus depth $z$.

Perhaps the most striking feature in Fig. 4.17 is that the detection efficiency is enhanced relative to the ballistic case. This can be explained by the fact that in a scattering medium, all the emitted light - including the light emitted in the forward direction - has a chance of being collected. It can also be seen that this enhancement persists to depths significant larger than $1100 \mu \mathrm{m}$ (linear extrapolation of the trace in Fig. 4.17 suggests $\sim 1320 \mu \mathrm{m}$ ) and attains its maximum (60\% above the ballistic level) at about $800 \mu \mathrm{m}(\sim 10$ scattering mean-free-path lengths). These findings are in line with recently published data obtained for anisotropic scattering media with smooth surfaces using Monte Carlo techniques, ${ }^{19}$ which indicate that this enhancement persists down to a depth about equal to the objective's effective field-ofview radius. In our case, the effective field-of-view radius for the objective used (Olympus XLUMPlanFl 20x/0.95W) is $1295 \mu \mathrm{m},{ }^{20}$ which is close to the maximum depth of enhanced detection efficiency suggested by our measurements $(1320 \mu \mathrm{m})$.

Summarising the findings of this section: we have shown, that a) the fluorescence detection efficiency for the out-of-focus volume scales roughly linearly with depth for depths smaller than the focus depth $(s \leq w . d$.$) ; b) the fluorescence detection efficiency for the focal volume is$ significantly enhanced compared to non-scattering media and is roughly constant out to depths of more than 14 scattering mean-free-path lengths. 
For the sake of completeness we note, that the results obtained above are only valid as long as the effect of fluorophore self-absorption (absorption of its own fluorescence radiation) is negligible. In the following we will demonstrate that for the majority of biological applications of two-photon microscopy this is indeed the case.

\subsubsection{Fluorescence self-absorption (inner filter effect)}

Since most fluorophores, posses a certain overlap between their excitation and emission spectra, they can absorb their own fluorescence radiation. ${ }^{21}$ This means that a fluorescence photon emitted by a specific fluorophore has a significant probability of exciting other fluorophore molecules of the same species leading to subsequent emission of another fluorescence photon. This process of self-absorption is however characterized by a loss factor equal to the quantum efficiency $\varepsilon_{q}$ times the absorption coefficient $\alpha(\lambda)$ i.e. within a small distance $d x$ the number of photons decreases by a constant fraction $d n=-\varepsilon_{q} \alpha(\lambda) n d x$, which upon integration yields

$$
n(x, \lambda)=n(x=0, \lambda) e^{-\varepsilon_{q} \alpha(\lambda) x}
$$

In a scattering medium, a photon emitted at depth $z$ will undergo a random walk until it reaches the surface and escapes. In general, the length $R$ of a walk of $n$ subsequent steps defined by their components $x_{i}, y_{i}, z_{i}$ is given by

$$
R=\sqrt{\left(x_{1}+x_{2}+\ldots+x_{n}\right)^{2}+\left(y_{1}+y_{2}+\ldots+y_{n}\right)^{2}+\left(z_{1}+z_{2}+\ldots+z_{n}\right)^{2}}
$$

In a random walk, the probability of walking in any direction is equal (isotropic) and the number of steps is large. Under these conditions, the term under the root simplifies to

$$
R=\sqrt{n r^{2}}=\sqrt{n} r
$$

where $r$ is the average distance walked per step. Note, although the average distance advanced is only $\sqrt{n} r$ the actual distance walked is $n r$ which is given by

$$
n r=\frac{R^{2}}{r^{2}} r=\frac{R^{2}}{r} .
$$


For a photon emitted at depth $z$ below the surface of a highly scattering medium i.e. $l_{s} \ll z$ the average distance traveled when emerging at the surface is thus

$$
x=n l_{s}=\frac{z^{2}}{l_{s}} .
$$

Using $\alpha(\lambda)=\varepsilon_{a}(\lambda) C$ where $\varepsilon_{a}(\lambda)$ is the absorbance extinction coefficient and $C$ the fluorophore concentration, the average fluorescence falls as

$$
F(z)=\int F_{0}(\lambda) e^{-\varepsilon_{q} \varepsilon_{a}(\lambda) C \frac{z^{2}}{l_{s}}} d \lambda
$$

In order to estimate the fluorescence loss expected for typical experimental conditions in twophoton microscopy, we consider an EGFP-labeled brain sample $\left(\varepsilon_{a}=5.5 \cdot 10^{-6}(\mu M \mu m)^{-1}\right.$ at $\left.488 \mathrm{~nm}, \varepsilon_{q} \cong 0.6\right)^{22,23}$ with scattering mean-free-path length $l_{s}=71 \mu \mathrm{m}($ at $500 \mathrm{~nm})$ and average fluorophore concentration of $1 \mu \mathrm{M}^{\dagger}$.

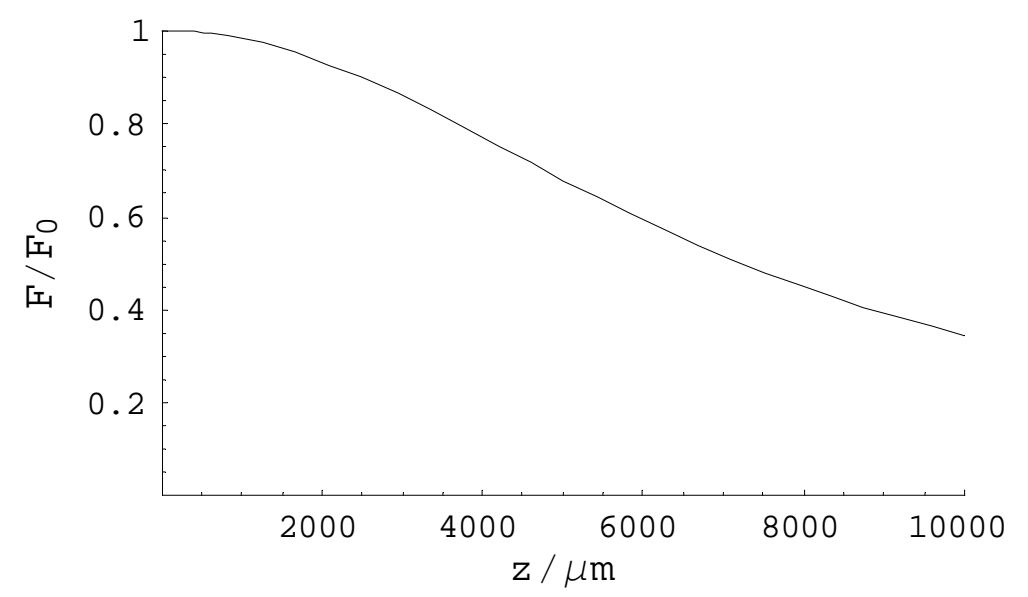

Fig. 4.18 Normalised fluorescence $\left(F / F_{0}\right)$ vs. source depth $(z)$

\footnotetext{
${ }^{\dagger}$ Assuming that the fluorophore concentration in strongly labeled neurons is about $100 \mu \mathrm{M}$ and the stained neuron volume fraction in a moderately stained sample i.e. $\sim 20$ neurons $/ \mathrm{nl}$, is about $1 \%$.
} 
For these parameters we have evaluated Eqn. 4.57 numerically (see Fig. 4.18) using the following approximations: the spectral characteristics of the fluorescence emission and absorbance extinction coefficient have been approximated by Gaussian distributions with maxima at 508 and $488 \mathrm{~nm}$ and 1/e widths of 11 and $13 \mathrm{~nm}$, respectively. While Gaussian distributions are in general inadequate for describing the EGFP absorption and emission spectra, they can provide a good approximation in the range of their spectral overlap (see Fig. 4.19), which is most crucial for the inner absorption effect. This can be seen from the following arguments: while for wavelengths below the short wavelength edge of the spectral overlap the contribution to the integral in Eqn. 4.57 is effectively zero due to the absence of fluorescence emission, for wavelengths above the long wavelength edge, absorption is negligible and the contribution to the integral in Eqn. 4.57 independent of the actual course of the fluorescence emission spectrum.

As can be seen from Fig. 4.18, for typical imaging depths in two-photon microscopy only small effects of inner absorption are expected i.e. for a fluorescent source e.g. a labeled neuron at a depth of $600 \mu \mathrm{m}$, the total fluorescence emerging from the sample surface, will be reduced by only $0.7 \%$. Significant effects $(>10 \%)$ are not expected before reaching depths as large as $2500 \mu \mathrm{m}$. The loss in fluorescence due to self-absorption by the fluorophore can thus be generally neglected.

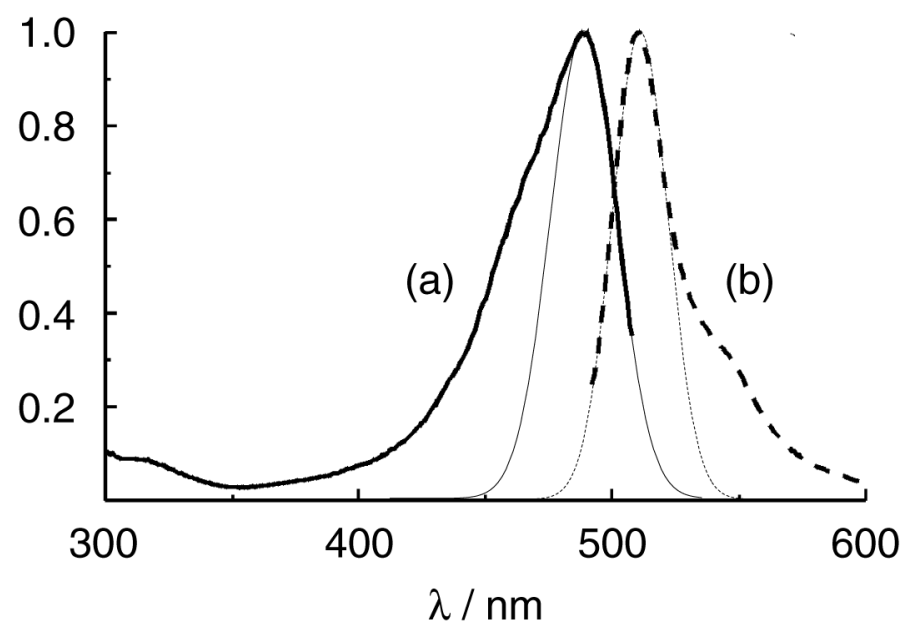

Fig. 4.19 Absorption and emission spectrum of EGFP adapted from Tsien ${ }^{23}$ and Gaussian distributions (thin solid lines) used for approximation of their central parts (overlapping region). 


\subsection{The fundamental two-photon imaging-depth limit}

In order to maintain a constant signal strength, i.e. constant focal excitation intensity (assuming depth-independent collection efficiency), the incident power needs to be increased exponentially with depth. Since this leads to an increase in out-of-focus fluorescence, the imaging-depth is fundamentally limited even if unlimited excitation power is available. This fundamental imaging-depth limit is given by the depth at which the focal fluorescence signal falls below the photon shot-noise level $\left(\sqrt{F_{S}+F_{B}}\right.$, in photon units). This definition, however, depends on imaging and tissue parameters as excitation power, fluorophore staining strength and statistics, excitation and detection efficiency, pixel dwell time, etc. and does therefore not provide a very useful number. Less dependent on the imaging parameters is the depth where the out-of-focus fluorescence signal equals the focal fluorescence signal

$$
\frac{F_{S}}{F_{B}}=\frac{\delta C_{S} \int_{J_{f}} \int_{-\infty}^{\infty} I_{\text {ball. }}^{2}(\rho, z, t) \mathrm{d} t \mathrm{~d} V}{\delta C_{B} \int_{V_{o o f}}^{\infty} \int_{-\infty}^{\infty}\left(I_{\text {scat. }}(\rho, z, t)+I_{\text {ball. }}(\rho, z, t)\right)^{2} \mathrm{~d} t \mathrm{~d} V}=1
$$

Whether or not imaging beyond that depth is possible, depends now, however, on the signalto-noise ratio. We have to consider, furthermore, that even for samples that are stained uniformly in a statistical sense the actual fluorophore concentration will be spatially inhomogeneous. While this does not affect the calculation of the background fluorescence, which essentially depends only on the average fluorophore concentration, the peak focal fluorescence increases as the inhomogeneity increases. While the exact amount of this increase depends on the staining statistics we can use, as an approximation, the concept of the 'stained volume fraction' $(l)$, which can be pictured as uniformly stained sub-volumes (with a combined volume that is $l$ times the total volume) that are large enough to contain the entire focal volume (the size necessary to meet this condition does depend on the NA; see section 4.2.3). This implies that

$$
C_{B}=\imath C_{S}=\frac{V_{\text {stained }}}{V_{0}} C_{S} .
$$

The maximum imaging depth is then determined by finding the $z$ for which 


$$
\int_{f} \int_{-\infty}^{\infty} I_{\text {ball. }}^{2}(\rho, z, t) \mathrm{d} t \mathrm{~d} V=\imath \int_{V_{\text {oof }}} \int_{-\infty}^{\infty}\left(I_{\text {scat. }}(\rho, z, t)+I_{\text {ball. }}(\rho, z, t)\right)^{2} \mathrm{~d} t \mathrm{~d} V .
$$

For a spherical focal volume with radius $0.5 \mu \mathrm{m}$ and the parameters given above, we have solved Eqn. 4.60 numerically. Figure 4.20 shows the depth limit $z_{\max }$ as a function of NA for various stained volume fractions $l$.

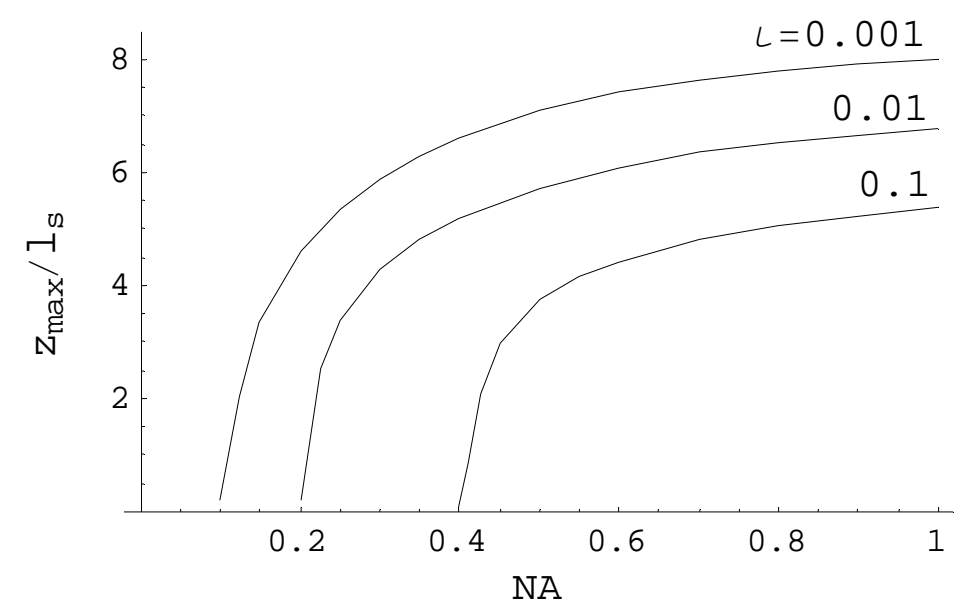

Fig. 4.20 Maximum two-photon imaging depth as a fucntion of effective NA for various stained volume fractions $l=0.001,0.01,0.1$.

The maximum imaging depth increases as the stained volume fraction is reduced and for large NA's depends roughly logarithmically on the stained volume fraction, with an increase by about one scattering mean-free-path length for a reduction of $l$ by a factor of $1 / 6$. This can be seen when plotting the maximum imaging depth as a function of stained volume fraction giving a linear trend. When comparing this expectation with our measurements (see Fig. 4.21) we found qualitative agreement. However, in terms of absolute values, the measured maximum imaging depths fall about 1 scattering mean-free-path length short of the expectation. This can be due to several factors. First, while the generation of out-of-focus fluorescence is rather insensitive to beam aberrations, in the focus they will lead to a spread in the PSF (see section 4.2.1) and thus reduce the focal excitation efficiency, corresponding to a constant negative shift in the maximum imaging depth in Fig. 4.21. Second, as shown in section 4.3, the detection efficiency for focal and out-of-focus fluorescence is different and generally depends on imaging depth. Due to the lack of reliable data over the entire range of interest, we did not yet incorporate any spatial dependence of the detection efficiency into our 
model. However, preliminary data on this subject (see section 4.3) suggest, that the ratio of focal to out-of-focus detection efficiency increases with depth. Since this corresponds to a stronger decay of the solid line in Fig. 4.21, it may account for the different slopes of measurement and expectation.

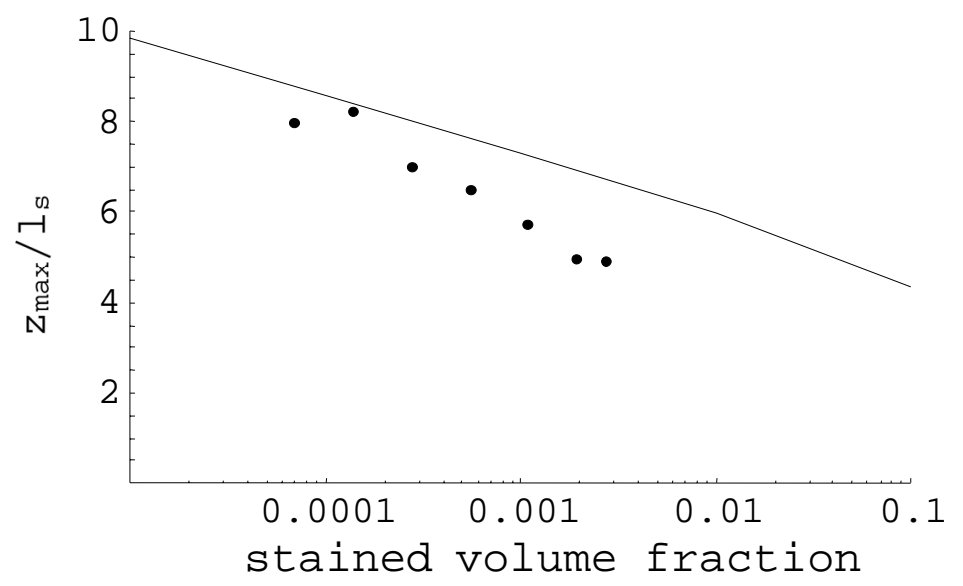

Fig. 4.21 Two-photon imaging-depth limit (expressed as multiples of the scattering mean-free-path length) vs. stained volume fraction. Solid line shows the theoretical course for NA $=0.6$; dots are measured values using a NA of 0.6 .

\subsection{Conclusions}

Among the various factors determining the fundamental imaging-depth limit in two-photon microscopy the most prominent are the excitation numerical aperture and the specimens staining properties. In general, the largest imaging depths can be achieved by using the largest excitation numerical aperture and the lowest out-of-focus volume staining strength. Of course, this can only be fully exploited by maximising the detection efficiency. However, although the large detection efficiency is at the origin of the superiority of two-photon microscopy over one-photon techniques, in the case of very deep imaging, it can become counterproductive because out-of-focus fluorescence is similarly well detected. For deep imaging it might therefore be beneficial to develop detection schemes that enable differentiation between focus and out-of-focus fluorescence and, thereby, allow suppression of the latter. One example of such a scheme is presented and discussed in chapter 5 . 


\section{References}

1. P. Theer and W. Denk, "On the fundamental imaging-depth limit in two-photon microscopy," in Femtosecond Laser Applications in Biology, Volume 5463 (SPIE, 2004), in press.

2. H. Kogelnik and T. Li, "Laser Beams and Resonators," Applied Optics 5(10), 1550-\& (1966).

3. A. E. Siegman, Lasers (University Science Books, Mill Valley, 1986), p. Chap. 17.

4. L. D. Dickson, "Characteristics of a Propagating Gaussian Beam," Applied Optics 9(8), 1854-\& (1970).

5. H. E. Keller, "Objective Lenses for Confocal Microscopy," in Handbook of Biological Confocal Microscopy, J. B. Pawley, ed. (Plenum Press, New York, 1995).

6. R. Juskaitis, "Characterizing High Numerical Aperture Microscope Objective Lenses," in Optical Imaging and Microscopy, P. Torok and F.-J. Kao, eds. (Springer, Berlin, 2003).

7. R. R. Birge, "2-Photon Spectroscopy of Protein-Bound Chromophores," Accounts of Chemical Research 19(5), 138-146 (1986).

8. J. Mobley and T. Vo-Dinh, "Optical Properties of Tissue," in Biomedical Photonics Handbook, V.-D. T., ed. (CRC Press, Boca Raton, 2003).

9. H. C. Vandehulst and G. W. Kattawar, "Exact Spread Function for a Pulsed Collimated Beam in a Medium with Small-Angle Scattering," Applied Optics 33(24), 5820-5829 (1994).

10. S. Kumar, K. Mitra, and Y. Yamada, "Hyperbolic damped-wave models for transient light-pulse propagation in scattering media," Applied Optics 35(19), 3372-3378 (1996).

11. C. M. Blanca and C. Saloma, "Efficient analysis of temporal broadening of a pulsed focused Gaussian beam in scattering media," Applied Optics 38(25), 5433-5437 (1999).

12. R. F. Lutomirski, A. P. Ciervo, and G. J. Hall, "Moments of Multiple-Scattering," Applied Optics 34(30), 7125-7136 (1995).

13. J. W. McLean, J. D. Freeman, and R. E. Walker, "Beam spread function with time dispersion," Applied Optics 37(21), 4701-4711 (1998).

14. V. I. Haltrin, "One-parameter two-term Henyey-Greenstein phase function for light scattering in seawater," Applied Optics 41(6), 1022-1028 (2002).

15. L. C. Henyey and J. L. Greenstein, "Diffuse radiation in the Galaxy," Astrophysics Journal 93, 70-83 (1941). 
16. J. P. Ying, F. Liu, and R. R. Alfano, "Spatial distribution of two-photon-excited fluorescence in scattering media (vol 38, pg 224, 1999)," Applied Optics 38(10), 21512151 (1999).

17. H. J. Koester, D. Baur, R. Uhl, and S. W. Hell, "Ca2+ fluorescence imaging with picoand femtosecond two-photon excitation: Signal and photodamage," Biophysical Journal 77(4), 2226-2236 (1999).

18. J. D. Jackson, Classical Electrodynamics, third ed. (John Wiley \& Sons, Inc., New York, 1999).

19. E. Beaurepaire and J. Mertz, "Epifluorescence collection in two-photon microscopy," Applied Optics 41(25), 5376-5382 (2002).

20. M. Oheim, E. Beaurepaire, E. Chaigneau, J. Mertz, and S. Charpak, "Two-photon microscopy in brain tissue: parameters influencing the imaging depth," Journal of Neuroscience Methods 111(1), 29-37 (2001).

21. S. Udenfriend, Fluorescence Assay in Biology and Medicine (Academic Press, New York, 1969).

22. G. H. Patterson, S. M. Knobel, W. D. Sharif, S. R. Kain, and D. W. Piston, "Use of the green fluorescent protein and its mutants in quantitative fluorescence microscopy," Biophysical Journal 73(5), 2782-2790 (1997).

23. R. Y. Tsien, "The green fluorescent protein," Annual Review of Biochemistry 67, 509544 (1998). 


\section{Strategies for extending the imaging depth beyond the depth}

\section{limit}

\subsection{Introduction}

In chapter 3 and 4 we have shown that the imaging depth in two-photon fluorescence microscopy is fundamentally limited by the generation of out-of-focus fluorescence. However, I would like to emphasis that this limit pertains only to the most commonly used form of two-photon fluorescence microscopy as introduced by Denk et al. ${ }^{1}$ (see chapter 2). In confocal two-photon fluorescence microscopy ${ }^{2}$ for example out-of-focus fluorescence is virtually removed from the detection path by placing a pinhole in front of the detector, which acts as a spatial filter selecting unscattered emission from the focal plane. Due to their inefficient use of scattered focal fluorescence, confocal techniques can, however, only be applied at shallow depths where out-of-focus fluorescence is usually not a problem.

Two-photon imaging beyond the fundamental depth-limit is thus possible if excitation and/or detection schemes could be found that allow at least partial differentiation between focus and out-of-focus fluorescence and suppression of the latter.

In the following we will investigate the feasibility of a detection scheme that uses the fact that the majority of out-of-focus fluorescence light is generated close to the surface i.e. within the first two scattering mean-free-path lengths. Because, although out-of-focus and focus fluorescence light is in general indistinguishable, the widths of their intensity distribution at the sample surface are different. While fluorescence light generated in the focal volume will undergo a large number of scattering events before leaving the sample, most of the out-offocus fluorescence light that is initially directed towards the sample surface will leave unscattered or after only a few scattering events. The out-of-focus fluorescence distribution $\left(F_{o o f}\right)$ at the sample surface will therefore, in part, resemble the squared intensity distribution of the incident excitation light which, using Eqn. 4.7, is given by

$$
F_{\text {oof }}(\rho)=I^{2}(\rho)=\left[\frac{2 P(t)}{\pi w^{2}(z=0)}\right]^{2} e^{-\frac{4 \rho^{2}}{w^{2}(z=0)}}
$$


The surface fluorescence distribution of the focus fluorescence has been derived in section 4.3 and is given by

$$
F_{f}(\rho)=\frac{Q}{2 \pi} \frac{z_{0}}{\left(\rho^{2}+z_{0}^{2}\right)^{3 / 2}}
$$

Unfortunately these distributions overlap. Hence, any suppression of the out-of-focus fluorescence using spatial filtering will inevitably reduce the focal fluorescence signal. However, this may still be beneficial if it leads to a gain in the signal(focus)-tobackground(out-of-focus fluorescence) ratio.

As the most simple spatial filter we consider an opaque disc placed at the image of the sample surface in the detection path. The signal-to-background ratio as a function of effective disc $\operatorname{radius}^{\dagger}(R)$ is then given by

$$
\frac{S}{B}=\frac{\int_{0}^{\infty} F_{f}(\rho) \varepsilon(\rho) 2 \pi \rho \mathrm{d} \rho-\int_{0}^{R} F_{f}(\rho) \varepsilon(\rho) 2 \pi \rho \mathrm{d} \rho}{\int_{0}^{\infty} F_{\text {oof }}(\rho) \varepsilon(\rho) 2 \pi \rho \mathrm{d} \rho-\int_{0}^{R} F_{\text {oof }}(\rho) \varepsilon(\rho) 2 \pi \rho \mathrm{d} \rho},
$$

where $\varepsilon(\rho)$ is the collection efficiency (in general a function of radial distance to the optical axis). For most objectives, the collection efficiency is roughly constant over their nominal field of view but decreases rapidly with increasing distance beyond that. ${ }^{3}$ In cases where the fluorescence distribution is much smaller than the objectives nominal field of view, Eqn. 5.3 simplifies to

$$
\frac{S}{B}=\frac{\pi Q}{P^{2}} \frac{z_{0} w(z=0)}{\sqrt{R^{2}+z_{0}^{2}}} e^{\frac{4 R^{2}}{w^{2}(z=0)}}
$$

In most situations, however, the fluorescence distribution (mainly that of the focus fluorescence) is significantly wider than the objective's nominal field of view and Eqn. 5.3 needs to be evaluated individually. The gain in two-photon imaging depth expected from an increase in the signal to background ratio can be calculated using Eqn. 4.6.

\footnotetext{
${ }^{\dagger}$ in order to block light from a circular sample surface area with radius $R$ requires an opaque disc at the image plane with radius $\mathrm{M}\left(z_{0}\right) R$, where $\mathrm{M}\left(z_{0}\right)$ is the transverse magnification of the detection optics which is a function of focal depth.
} 


\subsection{Methods and results}

\subsubsection{Surface fluorescence intensity distributions}

In order to test whether or not spatial filtering could increase the signal-to-background ratio and thus the two-photon imaging depth beyond the limit described in the previous chapter we have measured the fluorescence intensity distributions for the focal and out-of-focus fluorescence for different focus depths using a cooled CCD-camera (TEA/CCD-512-TKB-1 with controller ST-138S, both Princeton Instruments Inc.). The operating temperature of the CCD-chip was set to $-20{ }^{\circ} \mathrm{C}$. The sample surface was imaged onto the CCD-chip through the objective (Olympus XLUMPlanFl 20x/0.95W) and the collection lens $(f=100 \mathrm{~mm}$ ) of the two-photon microscope described in section 3.4.1. In order to maintain the normal imaging mode of the microscope, a 45 degree mirror was inserted between collection lens and PMT that could be slid in and out the detection path switching between PMT and CCD-camera detection. An additional barrier filter (BG38, Schott) was placed in front of the CCD.

The collection efficiency of the used objective as a function of radial distance to the optical axes was taken from published data. ${ }^{3}$

\section{Out-of-focus fluorescence distribution}

For the measurements of the out-of-focus fluorescence distribution we used a phantom with $93 \mathrm{~nm}$ yellow-green (490/515) fluorescent beads (Molecular Probes Inc.) at a concentration of $2.52 \cdot 10^{11}$ beads $/ \mathrm{ml}$ embedded in a brain tissue phantom containing $0.992 \mu \mathrm{m}$ non-fluorescent polystyrene scattering beads (Polyscience Inc.) at a concentration of $5.3 \cdot 10^{9}$ beads $/ \mathrm{ml}$ (see also section 3.4.2).

Images of the fluorescence distribution at the sample surface were acquired for focal depths up to $1100 \mu \mathrm{m}$ at increments of $100 \mu \mathrm{m}$. In order to minimise fluorescence contributions from within the focal volume and neighbouring regions focus positions were selected for maximum separation to any fluorescent bead.

$1 / \mathrm{e}^{4}$ widths $w(z=0)$ of the distributions were determined from collection-efficiency corrected best fits to the two-dimensional two-photon fluorescence intensity distributions using Eqn. 5.1 $1^{\dagger}$ and are shown in Fig. 5.1.

\footnotetext{
${ }^{\dagger}$ considering no temporal dependence of the excitation power
} 


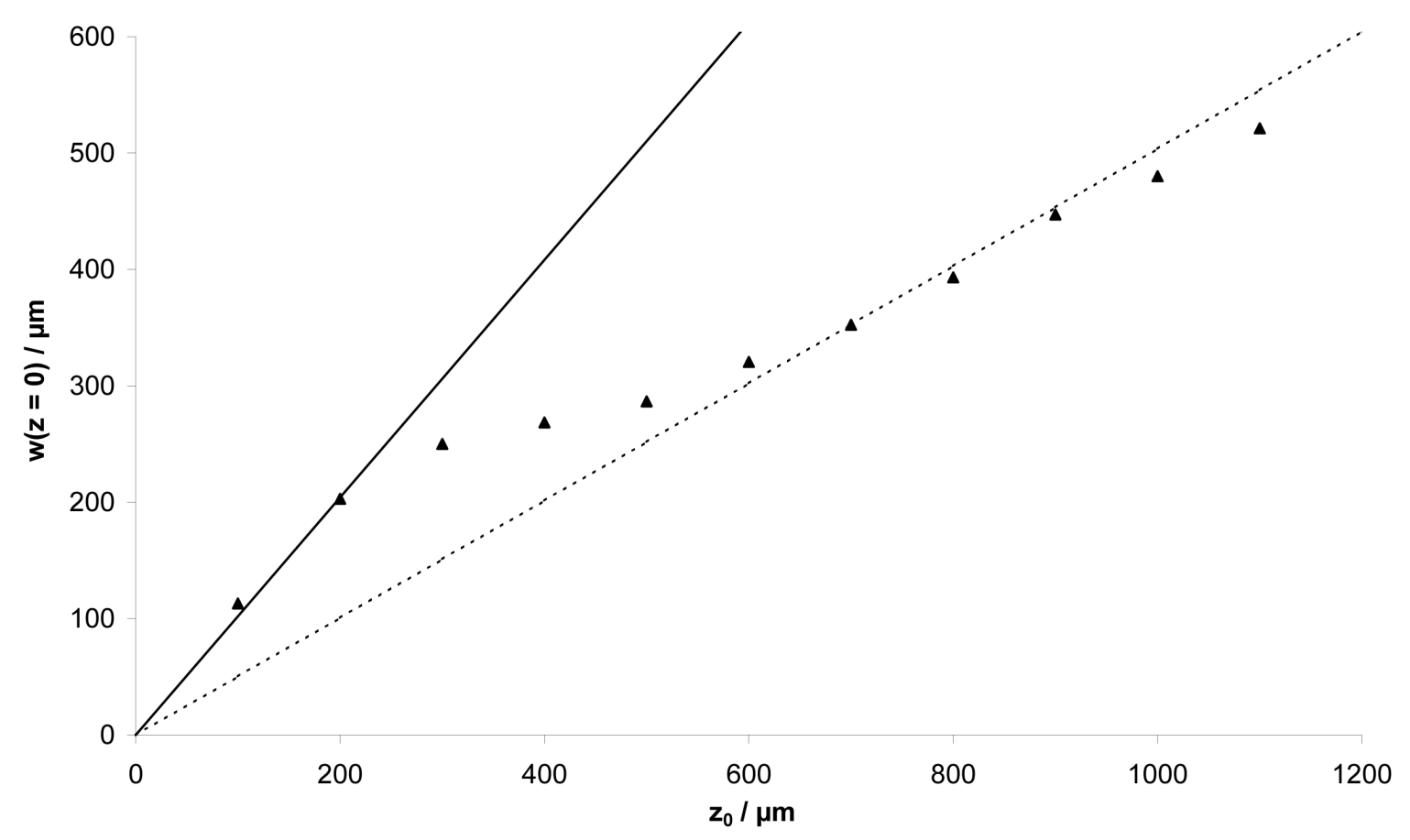

Fig. $5.1 \quad 1 / \mathrm{e}^{4}$ width of the out-of-focus fluorescence distribution at the surface of a brain tissue phantom (see text) as a function of focus depth $z_{0}$. The solid line corresponds to the width of the surface cross-section of a full NA light cone $\left(w=z_{0} \tan \left[\theta_{N A=0.95}\right]=1.021 z_{0}\right)$ and the dashed line to the $1 / \mathrm{e}^{2}$ width of the excitation beam $\left(w=\left.z_{0} \tan [\arcsin (\beta N A / n)]\right|_{\beta=0.63, N A=0.95}=0.504 z_{0}\right)$.

For small focus depths, the width of the fluorescence distribution was found to closely match that of the full NA light cone which is expected since for small focus depths, the fluorescence is predominantly generated near the focus and most of the fluorescence light initially directed towards the sample surface will leave unscattered. It will thus appear to be emitted from a point-source. Increasing the focus depth, the fluorescence will be generated in essentially two separate volumes (see Fig. 4.12), one near the focus and the other close to the sample surface whereby the latter resembles essentially the squared intensity distribution of the incident excitation light. At depths much larger than the scattering mean-free-path length, fluorescence is generated predominantly near the surface and the $1 / \mathrm{e}^{4}$ width of the surface fluorescence distribution will asymptotically approach the $1 / \mathrm{e}^{2}$ width of the incident excitation light (see Fig. 5.1). 


\section{Focus fluorescence distribution}

In order to obtain a sufficient large focus fluorescence signal, for the measurement of the focus fluorescence distribution, we used large fluorescent beads with a diameter of $9.008 \mu \mathrm{m}$ at a concentration of $6.8 \cdot 10^{5}$ beads $/ \mathrm{ml}$ embedded in a brain tissue phantom containing 0.992 $\mu \mathrm{m}$ non-fluorescent polystyrene scattering beads at a concentration of $3.8 \cdot 10^{9} \mathrm{beads} / \mathrm{ml}$ and $1.011 \mu \mathrm{m}$ fluorescent polystyrene beads (all beads from Polyscience Inc.) at a concentration of $1.59 \cdot 10^{9}$ beads $/ \mathrm{ml}$ as described in section 3.4.2.

Two-dimensional two-photon fluorescence intensity distributions were determined from two measurements. First, an image of the sample surface was taken with the focus centered on one of the $9 \mu \mathrm{m}$ fluorescent beads thus acquiring an image of the combined distribution of focus and out-of-focus fluorescence. Second, another image was acquired with the focus moved just out of the fluorescent bead (total lateral focus shift $<20 \mu \mathrm{m}$ ) by applying an offset to the laser scan mirrors thus acquiring an image of the out-of-focus fluorescence distribution alone. Since such a small movement of the focus position does not lead to a significant change in the outof-focus volume respective fluorescence, the focal fluorescence distribution can be determined by subtraction of the second image from the first. The out-of-focus fluorescence distribution can be obtained from the second image by simple background subtraction. Half widths at half maximum $\left(r_{H W H M}\right)$ were determined from collection-efficiency corrected best fits using Eqn. 5.2. According to Eqn. 5.2, the $r_{H W H M}$ depends linearly on the source depth and is determined by

$$
\frac{F_{F}\left(r_{H W H M}\right)}{F_{F}(r=0)}=\frac{z_{0}^{3}}{\left(r_{H W H M}^{2}+z_{0}^{2}\right)^{3 / 2}}=0.5
$$

which reduces to

$$
r_{H W H M}=z_{0} \sqrt{2^{2 / 3}-1} \cong 0.766 z_{0}
$$

In the limit of large focus depths Eqn. 5.6 provides a fair approximation to the measured data (see Fig. 5.2). 


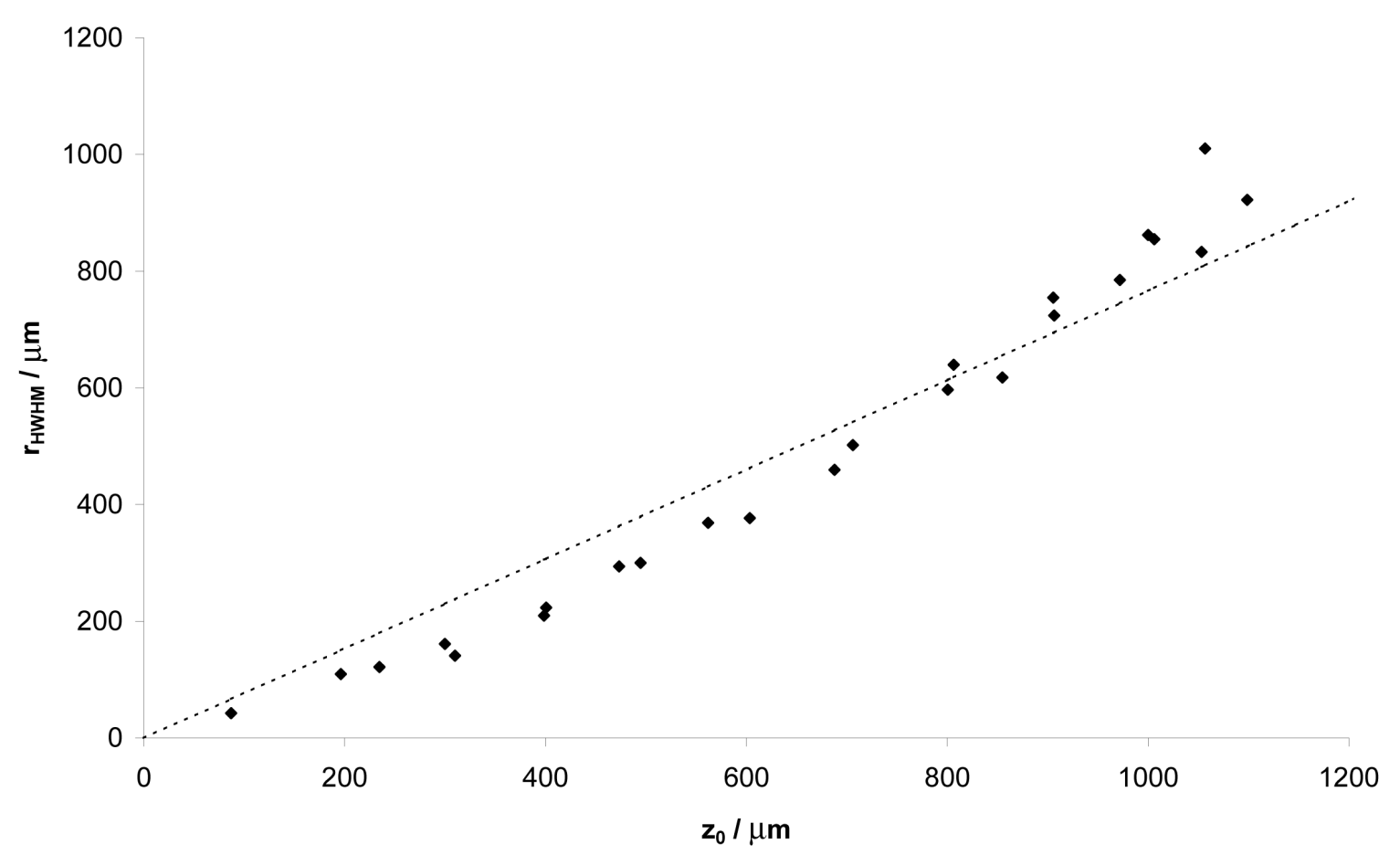

Fig. 5.2 $\quad r_{H W H M}$ of the focus fluorescence distribution at the surface of a brain tissue phantom (see text) as a function of focus depth $z_{0}$. The dashed line corresponds to the width expected for the diffuse case $\left(r_{H W H M} \cong 0.766 z_{0}\right)$.

While for small focus depths fits were still surprisingly good (all data points are derived from best fits with a regression coefficient $\mathrm{R}^{2}>0.97$ ) Eqn. 5.2 naturally overestimates the width of the fluorescence distributions for depths $<800 \mu \mathrm{m}$. In fact, at small focus depths we do not expect significant differences between the focal and out-of-focus surface fluorescence distribution since they both have their origin at volumes close to the focus. Increasing the depth, however, leads to a significant separation of these volumes resulting in different shapes and widths of their distributions, which in the limit of large depths are well described by theory (see Fig. 5.3). To illustrate this we have plotted the focus and out-of-focus surface fluorescence as a function of radial distance from the optical axes for a focus depth of $900 \mu \mathrm{m}$ (see Fig. 5.4). 


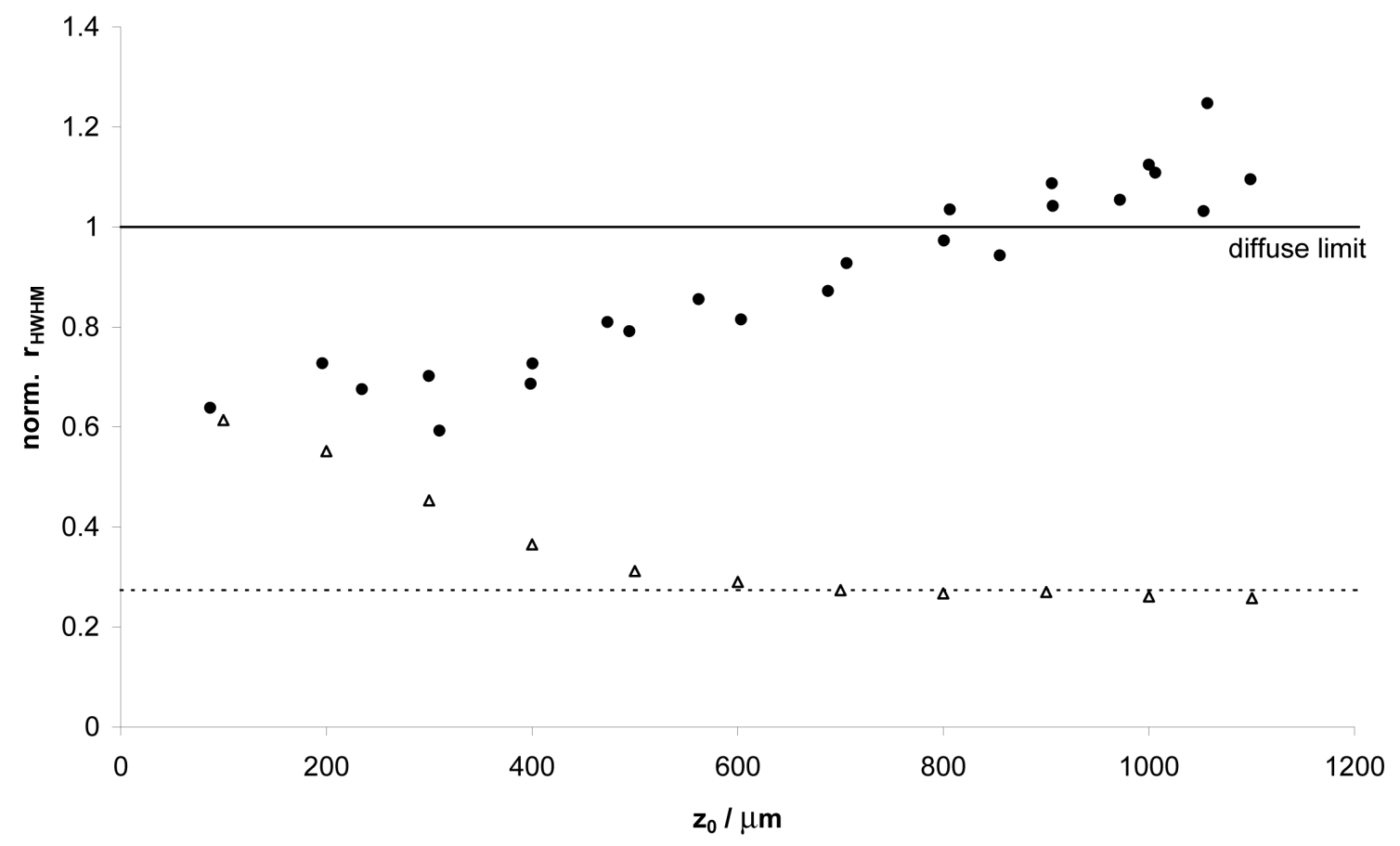

Fig. 5.3 Half width at half maximum $\left(r_{H W H M}\right)$ of focus (filled circles) and out-of-focus fluorescence distributions (triangles) at the surface of a brain tissue phantom (see text) normalised to the width expected for the diffuse case $\left(r_{H W H M}=0.766 z_{0}\right)$ as a function of focus depth $z_{0}$. The solid line corresponds to the width expected for the diffuse case and the dashed line to the HWHM of the surface cross-section of the excitation beam $\left(\mathrm{HWHM}=\sqrt{\ln [2] / 4} w \cong 0.209 z_{0}\right)$.

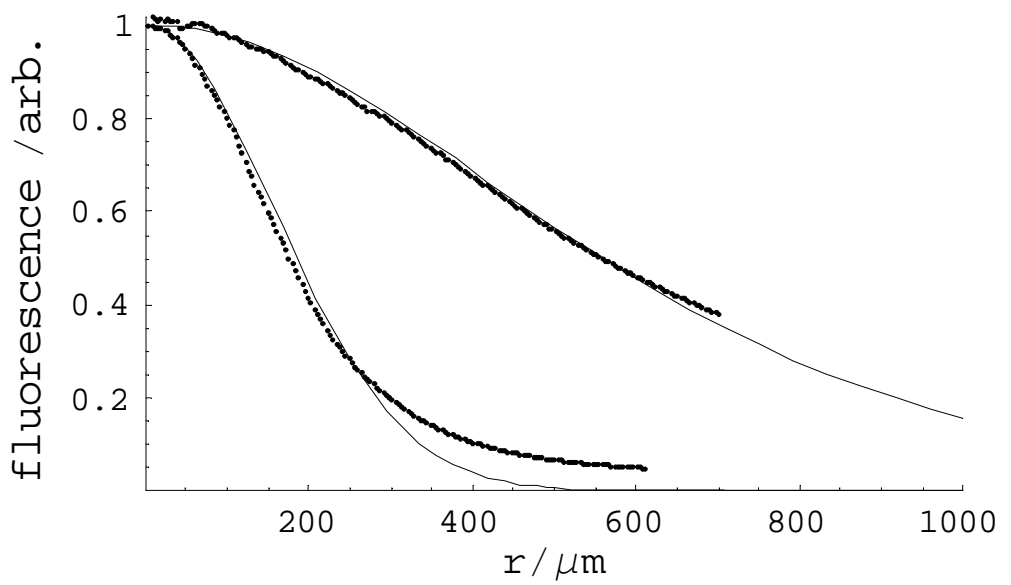

Fig. 5.4 Focus and out-of-focus fluorescence intensity distribution at the surface of a brain tissue phantom (see text) for a focus depth of $900 \mu \mathrm{m}$. Solid lines are expected from theory and calculated using Eqn. 5.1 and 5.2. 
While in the depth range of interest ( $>800 \mu \mathrm{m}$; around the current imaging depth limit), the widths of the measured distributions are well approximated by theory, we note that the shape of the out-of-focus fluorescence distribution deviates from the expected Gaussian shape showing significant larger wings, which are due to the portion of out-of-focus fluorescence light that is initially directed away from the sample surface. This light will, in general, leave the sample after a large number of scattering events with a diffuse distribution similar to that of the focal fluorescence. Hence, although the distributions of the focal and out-of-focus fluorescence seem to be sufficiently separated as to allow the signal-to-background ratio to be increased by suppressing the central part of the combined surface fluorescence distribution, the achievable gain will critically depend on the amount of diffuse out-of-focus fluorescence and the detection efficiency for the wings of its distribution. In fact, although the diffuse portion in the out-of-focus distribution measured at a focus depth of $900 \mu \mathrm{m}$ (see Fig. 5.4) seems quite small, spatial filtering i.e. suppressing the central part of the fluorescence distribution, will allow an increase in the $\mathrm{S} / \mathrm{B}$ ratio by no more than a factor of $2.25^{\dagger}$ (see Fig. 5.5). This corresponds to an increase in imaging depth of less than half a scattering mean-freepath length.

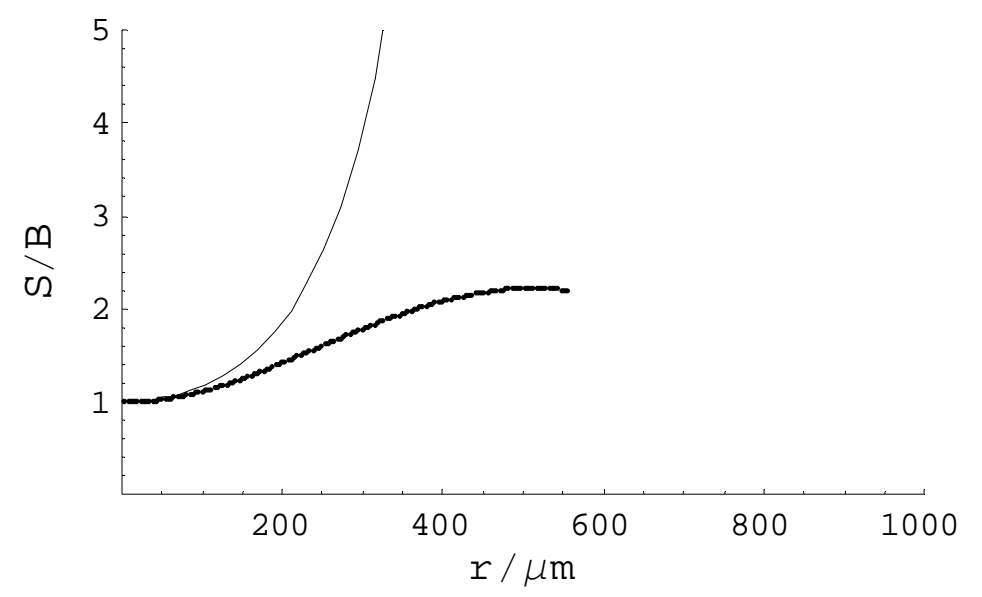

Fig. 5.5 Signal-to-background ratio as a function of radius of a circular obstruction centered at the optical axis focus and out-of-focus fluorescence intensity distribution at the surface of a brain tissue phantom (see text) for a focus depth of $900 \mu \mathrm{m}$. The maximum occurs for an obstruction radius of around $500 \mu \mathrm{m}$ at which the focal fluorescence signal is reduced to about $25 \%$. The solid line is from the theory (Eqn. 5.4).

\footnotetext{
${ }^{\dagger}$ when considering a detection efficiency of unity for $r \leq 600 \mu \mathrm{m}$ and zero for $r>600 \mu \mathrm{m}$
} 


\subsection{Conclusions}

The imaging depth in two-photon microscopy can be increased beyond the fundamental limit by departing from the concept of whole-field detection. As an example, we have shown that a more than 2-fold increase in the ratio of detected focus to out-of-focus fluorescence can be achieved by spatial filtering using an opaque disc in the detection path. While a substantial increase in the signal-to-background ratio is expected, the imaging depth is expected to increase by at most half a scattering mean-free-path length. In view of the complexity involved in retrofitting a two-photon microscope with a spatial filter as described above (size and position of the opaque disc need to be adapted for different imaging depths), the practical value of this approach seems rather limited. Nevertheless, even if the increase in imaging depth might be marginal increasing the signal-to-background ratio is desirable at any imaging depth since it increases the signal-to-noise ratio and thus allows for improved signal quantification and a reduction in excitation power (less photo damage).

\section{References}

1. W. Denk, J. H. Strickler, and W. W. Webb, "2-Photon Laser Scanning Fluorescence Microscopy," Science 248(4951), 73-76 (1990).

2. E. H. K. Stelzer, S. Hell, S. Lindek, R. Stricker, R. Pick, C. Storz, G. Ritter, and N. Salmon, "Nonlinear Absorption Extends Confocal Fluorescence Microscopy into the Ultra-Violet Regime and Confines the Illumination Volume," Optics Communications 104(4-6), 223-228 (1994).

3. M. Oheim, E. Beaurepaire, E. Chaigneau, J. Mertz, and S. Charpak, "Two-photon microscopy in brain tissue: parameters influencing the imaging depth," Journal of Neuroscience Methods 111(1), 29-37 (2001). 


\section{Summary}

This work was motivated by the need for high resolution two-photon imaging techniques that allow the study of deep layers of cortex in vivo. In the first part of this dissertation we show that the imaging depth in two-photon microscopy - previously restricted by the power current femto-second laser can provide - can be substantially increased by using optically amplified laser pulses. Using a regenerative amplifier as the excitation source we obtained images of stained vasculature and GFP-labeled neurons down to a depth of about $1000 \mu \mathrm{m}$ below the brain surface in the cortex of mice in vivo. Compared to what has been achieved previously, this is an increase of about two scattering mean-free-path lengths or $\sim 400 \mu \mathrm{m}$ in brain tissue. We also obtained functional signals down to a depth of $900 \mu \mathrm{m}$ measuring the motion of red blood cells in individual capillaries with a diameter of about $5 \mu \mathrm{m}$. In all cases, the maximum imaging depth was not limited by the available excitation power but by a loss in image contrast, which is the result of an increase in the amount of two-photon excited out-of-focus fluorescence. The reason for this is that in order to maintain a constant signal strength (focal fluorescence) the incident laser power needs to be increased exponentially with depth. For large depths, this exponential increase in power, although partially compensated by an increase in the beams cross section results in an overall increase in excitation intensity mainly near the sample surface eventually generating background fluorescence comparable to that produced in the focus. This sets a fundamental limit to the imaging depth that can be achieved in two-photon and, in fact, any order of multi-photon excitation microscopy.

A quantitative description of this behaviour is given in chapter 4. In particular, we have investigated the role of tissue properties and imaging parameters on the generation and detection of two-photon excited fluorescence and provide strategies for improving the imaging depth.

Among the various factors determining the fundamental imaging-depth limit in two-photon microscopy we have found that the excitation numerical aperture and the specimens staining properties are the most prominent. In general, the largest imaging depths can be achieved by using the largest excitation numerical aperture and the lowest out-of-focus volume staining strength. This can only be fully exploited by maximising the detection efficiency. But, although a large detection efficiency is one of the main advantages of two-photon microscopy 
over one-photon techniques, it can become somewhat disadvantageous at large imaging depths. This is because out-of-focus fluorescence is detected as well or better as focal fluorescence. We therefore propose detection schemes that enable partial differentiation of focus and out-of-focus fluorescence and allow suppression of the latter. One example of such a scheme utilising spatial filtering is presented. Although we can demonstrate that the imaging depth in two-photon microscopy can principally be increased beyond the fundamental limit using such schemes, their practical value is rather limited. The reason for this is that the fluorescence distributions of focus and out-of-focus fluorescence show a significant spatial as well as temporal overlap. Hence, suppression of out-of-focus fluorescence using spatial filtering comes at the cost of a reduced focal fluorescence thereby limiting the achievable gain in imaging depth. 


\section{Acknowledgements}

I would like to express my gratitude to all those who contributed to this work. In particular I would like to thank Prof. Winfried Denk for his guidance and ever surprising ideas on how to proceed or interpret the accomplished. I am grateful to Prof. Josef Bille for his efforts as referee of this dissertation.

I would like to thank Maz Hasan for all the in vivo preparations, Philipp Keller who did most of the measurements and data analysis presented in chapter 5, Dorine Keusters for fruitful discussions and comments on the dissertation, and Marcus Feierabend for sharing the experience of writing one. To Christa Hoerner-Ehm many thanks for easing all the administrative barriers. Thanks to Jürgen Tritthart and Michael Müller for lending their ears and hands to rein fractious electronics and computers. Special thanks to the staff of the machine shop for helpful comments on the design of mechanical parts and prompt and careful manufacture. To all my lab mates, thanks for taking your time to share mine.

The enlightening illustrations and much of the layout have been enthusiastically contributed by Johanna Berking. 


\section{Appendix}

\section{Electromagnetic wave in absorbing media}

Electromagnetic fields are represented by two basic field vectors called the electric vector $(\mathbf{E})$ and the magnetic induction (B). The behaviour of matter under the influence of electromagnetic fields is described by uniquely determined by Maxwell's equations

$$
\begin{gathered}
\nabla \cdot \mathbf{D}=\rho \\
\nabla \times \mathbf{H}=\mathbf{j}+\frac{\partial \mathbf{D}}{\partial t} \\
\nabla \times \mathbf{E}=-\frac{\partial \mathbf{B}}{\partial t} \\
\nabla \cdot \mathbf{B}=0
\end{gathered}
$$

relating the space and time derivatives of $\mathbf{E}, \mathbf{B}$, the electric current density $\mathbf{j}$, the electric displacement $\mathbf{D}$ and the magnetic vector $\mathbf{H}$ where $\rho$ is the electric charge density. For isotropic media characterised by their specific conductivity $(\sigma)$, dielectric constant $(\varepsilon)$ and magnetic permeability $(\mu), \mathbf{j}=\sigma \mathbf{E}, \mathbf{D}=\varepsilon \mathbf{E}$, and $\mathbf{B}=\mu \mathbf{H}$, the first two equations become

$$
\begin{gathered}
\nabla \cdot \mathbf{E}=\frac{1}{\varepsilon} \rho \\
\nabla \times \mathbf{B}=\mu \sigma \mathbf{E}+\frac{1}{c^{2}} \frac{\partial \mathbf{E}}{\partial t}
\end{gathered}
$$

where $c=1 / \sqrt{\mu \varepsilon}$.

Taking the temporal derivative of Eqn. A.2' gives

$$
\nabla \times \frac{\partial \mathbf{B}}{\partial t}=\mu \sigma \frac{\partial \mathbf{E}}{\partial t}+\frac{1}{c^{2}} \frac{\partial^{2} \mathbf{E}}{\partial t^{2}}
$$


Rewriting the left side using Eqn. A.3 and A.1' yields

$$
\nabla \times \frac{\partial \mathbf{B}}{\partial t}=\nabla \times \nabla \times \mathbf{E}=-\Delta \mathbf{E}+\nabla(\nabla \cdot \mathbf{E})=-\Delta \mathbf{E}+\nabla\left(\frac{1}{\varepsilon} \rho\right) .
$$

In the absence of sources $(\rho=0)$ Eqn. A.6 simplifies to

$$
\nabla \times \frac{\partial \mathbf{B}}{\partial t}=-\Delta \mathbf{E}
$$

which substituted in Eqn. A.5 yields the telegrapher equation

$$
\Delta \mathbf{E}+\mu \sigma \frac{\partial \mathbf{E}}{\partial t}+\frac{1}{c^{2}} \frac{\partial^{2} \mathbf{E}}{\partial t^{2}}=0
$$

We seek a solution in the form of a monochromatic wave which can be conveniently represented by a complex wavefunction

$$
\mathbf{E}(\mathbf{r}, t)=\mathbf{E}(\mathbf{r}) e^{\mathrm{i} \omega t}
$$

with a time independent complex amplitude $\mathbf{E}(\mathbf{r})$ given by

$$
\mathbf{E}(\mathbf{r})=A(\mathbf{r}) e^{\mathrm{i} \varphi(\mathbf{r})}
$$

where $\omega$ and $\varphi(\mathbf{r})$ are the angular frequency and phase respectively. Substituting Eqn. A.10. into the telegrapher equation, one obtains

$$
\left(\Delta+k^{2}-\mathrm{i} \mu \sigma \omega\right) \mathbf{E}(\mathbf{r})=0
$$

where $k=\omega / c=2 \pi n / \lambda$ is the wavenumber in the medium. For loss less media $(\sigma=0)$, Eqn. A.11 simplifies to the Helmholtz wave equation

$$
\left(\Delta+k^{2}\right) \mathbf{E}(\mathbf{r})=0
$$


In the next step we derive the analytical form for a Gaussian spherical wave called Gaussian beam. Consider first a damped spherical wave given by

$$
\mathbf{E}(r)=\frac{1}{r} e^{\mathrm{i}(\omega t-k r)} e^{-\delta r}
$$

Differentiating with respect to $t$ gives a factor i $\omega$ and differentiating with respect to $r$ a factor $(\delta+\mathrm{i} k)$. Hence substitution in Eqn. A.11 omitting the common factor yields

$$
\delta^{2}+2 \mathrm{i} k \delta-\mathrm{i} \mu \sigma \omega=0
$$

with the solutions

$$
\delta=-\mathrm{i} k \pm \sqrt{-k^{2}+\mathrm{i} \mu_{0} \sigma \omega}
$$

With $\omega=k c$ the root in Eqn. A.15 becomes $\sqrt{-k^{2}+\mathrm{i} \mu \sigma k c}=\sqrt{k} \sqrt{-k+\mathrm{i} \mu \sigma c}$ and in the case where $\mu \sigma \mathrm{c} \ll k$ i.e. the wave can penetrate much deeper than a wavelength, can be approximated giving

$$
\delta \cong-\mathrm{i} k+\sqrt{k}\left(\sqrt{-k}+\frac{\mathrm{i} \mu \sigma c}{2 \sqrt{-k}}\right)=-\mathrm{i} k+\sqrt{k}\left(\mathrm{i} \sqrt{k}+\frac{\mathrm{i} \mu \sigma c}{2 \mathrm{i} \sqrt{k}}\right)=\frac{\mu \sigma c}{2}
$$

For the spherical wave solution to the telegraphers equation one thus obtains

$$
\mathbf{E}(r)=\frac{1}{r} e^{-\mathrm{i} k r} e^{-\frac{\mu \sigma c}{2} r}
$$

Since the intensity of the wave is proportional to $E^{2}$, it decays twice as fast as the field. Hence the absorption coefficient of the medium for this case is given by

$$
\alpha=\mu \sigma c
$$

with dimensions $\left[\mu_{0} \sigma c\right]=\frac{V s}{A m} \frac{A}{V m} \frac{m}{s}=\frac{1}{m}$. Hence 


$$
\mathbf{E}(r)=\frac{1}{r} e^{-\mathrm{i} k r} e^{-\alpha r}
$$

Within the paraxial approximation - a frame sufficient to describe essentially all laser beam propagation problems of practical interest (for range of validity see for example Siegman ${ }^{1}$ ) the solution for the spherical wave given above can be approximated by a paraboloidal wave. One way of doing this is by using the Fresnel-approximation given $b y^{2}$

$$
r=\sqrt{x^{2}+y^{2}+z^{2}} \cong z+\frac{x^{2}+y^{2}}{2 z}=z+\frac{\rho^{2}}{2 z} .
$$

Substitution of Eqn. A.20 in Eqn. A.19 for the phase but only $z$ for the amplitude gives the paraboloidal wave

$$
\mathbf{E}(r)=\frac{1}{z} e^{-\mathrm{i} k z} e^{-\mathrm{i} k \frac{\rho^{2}}{2 z}} e^{-\frac{\alpha}{2} z} e^{-\frac{\alpha}{2} \frac{\rho^{2}}{2 z}}
$$

with complex amplitude

$$
A(r)=\frac{A_{0}}{z} e^{-\mathrm{i} k \frac{\rho^{2}}{2 z}} e^{-\frac{\alpha \rho^{2}}{22 z}}
$$

The Gaussian beam as one of the most widely used optical beams can be derived from the paraboloidal wave by the introduction of complex source point coordinates. Replacing $z$ with $z+j z_{r}$ in Eqn. A.22 gives

$$
A(r)=\frac{A_{0}}{z+\mathrm{i} z_{r}} e^{-\mathrm{i} k \frac{\rho^{2}}{2\left(z+\mathrm{i} z_{r}\right)} e^{-\frac{\alpha}{2} 2\left(z+\mathrm{i} z_{r}\right)}}=\frac{A_{0}}{z+\mathrm{i} z_{r}} e^{-\rho^{2} \frac{1}{w^{2}(z)}} e^{-\mathrm{i} \rho^{2} \frac{\mathrm{k}}{2 R(z)}} .
$$

where $w(z)$ is measure of the beam width defined as the radial distance at which the field has dropped to $1 / \mathrm{e}(\sim 0.37)$ times that on axes given by

$$
w(z)=\sqrt{\frac{2\left(z^{2}+z_{r}^{2}\right)}{k z_{r}+\frac{\alpha}{2} z}}=\sqrt{\frac{4\left(z^{2}+z_{r}^{2}\right)}{2 k z_{r}+\alpha z}}=2 \sqrt{\frac{\lambda\left(z^{2}+z_{r}^{2}\right)}{4 \pi n z_{r}+\lambda \alpha z}}
$$


and $\mathrm{R}(\mathrm{z})$ the wavefront radius of curvature given by

$$
R(z)=\frac{k\left(z^{2}+z_{r}^{2}\right)}{k z-\frac{\alpha}{2} z_{r}}=\frac{\left(z^{2}+z_{r}^{2}\right)}{z-\alpha \lambda z_{r} /(4 n \pi)}
$$

The parameter $z_{r}$ is the Rayleigh range defined by the axial distance from the beam waist (@ $\mathrm{z}$ $=0$ ) for which the beam width is a factor of $\sqrt{2}$ larger than its minimal width at the waist $w_{0}=$ $w(z=0)$.

Without absorption, the beam width and radius of curvature reduce to

$$
\begin{gathered}
w(z)=\sqrt{\frac{2\left(z^{2}+z_{r}^{2}\right)}{k z_{r}}}=\sqrt{\frac{2 \lambda\left(z^{2}+z_{r}^{2}\right)}{2 \pi n z_{r}}}=\sqrt{\frac{\lambda}{\pi n} z_{r}} \sqrt{1+\frac{z^{2}}{z_{r}^{2}}}=w_{0} \sqrt{1+\frac{z^{2}}{z_{r}^{2}}}, \\
R(z)=\frac{k\left(z^{2}+z_{r}^{2}\right)}{k z}=\frac{\left(z^{2}+z_{r}^{2}\right)}{z}=z\left[1+\left(\frac{z_{r}}{z}\right)^{2}\right]
\end{gathered}
$$

where the beam waist is given by

$$
w_{0}=w(z=0)=\sqrt{\frac{\lambda z_{r}}{n \pi}} .
$$

Hence the complex amplitude of the Gaussian beam simplifies to

$$
\mathbf{E}(r)=\frac{A_{0}}{z+\mathrm{i} z_{r}} e^{-\frac{\alpha}{2} z-\frac{\rho^{2}}{w^{2}(z)}} e^{-\mathrm{i} k z-\mathrm{i} k \frac{\rho^{2}}{2 R(z)}}
$$

and the intensity $I(r)=|\mathbf{E}(r)|^{2}$ is given by

$$
I(r)=I(z, \rho)=\frac{P}{z^{2}+z_{r}^{2}} e^{-\alpha z-\frac{2 \rho^{2}}{w^{2}(z)}}
$$

The total optical power carried by the beam is 


$$
P(z)=\int_{0}^{\infty} I(z, \rho) 2 \pi \rho \mathrm{d} \rho=\frac{2 \pi \lambda P}{4 n \pi z_{r}+\lambda \alpha z} e^{-\alpha z}
$$

normalisation of Eqn. A.28 to $P(z=0)=P_{0}$ yields

$$
I(z, \rho)=\frac{2 n z_{r} P_{0}}{\lambda\left(z^{2}+z_{r}^{2}\right)} e^{-\alpha z} e^{-\frac{2 \rho^{2}}{w^{2}(z)}} .
$$

Note, although Eqn. A.30 has been derived for absorbent media it describes correctly the intensity distribution of unscattered light (ballistic fraction) in scattering media. However, scattered light, in contrast to absorbed light is not completely lost from the system but lost from a beam travelling in a certain direction - contributing to others. Also, light can get back into the beam by multiple scattering contributing to its initial direction. Hence, the intensity measured in a particular experiment will include contributions from both scattered and unscattered light.

\section{References}

1. A. E. Siegman, Lasers (University Science Books, Mill Valley, 1986), p. Chap. 17.

2. B. E. A. Saleh and M. C. Teich, Fundamentals of Photonics (John Wiley \& Sons, Inc., New York, 1991). 